\title{
Upscaling a Model for the Thermally-Driven Motion of Screw Dislocations
}

\author{
T. HudSON(1) \\ Communicated by M. ORTIZ
}

\begin{abstract}
We formulate and study a stochastic model for the thermally-driven motion of interacting straight screw dislocations in a cylindrical domain with a convex polygonal cross-section. Motion is modelled as a Markov jump process, where waiting times for transitions from state to state are assumed to be exponentially distributed with rates expressed in terms of the potential energy barrier between the states. Assuming the energy of the system is described by a discrete lattice model, a precise asymptotic description of the energy barriers between states is obtained. Through scaling of the various physical constants, two dimensionless parameters are identified which govern the behaviour of the resulting stochastic evolution. In an asymptotic regime where these parameters remain fixed, the process is found to satisfy a Large Deviations Principle. A sufficiently explicit description of the corresponding rate functional is obtained such that the most probable path of the dislocation configuration may be described as the solution of Discrete Dislocation Dynamics with an explicit anisotropic mobility which depends on the underlying lattice structure.
\end{abstract}

\section{Introduction}

Dislocations are topological line defects whose motion is a key factor in the plastic behaviour of crystalline solids. After their existence was hypothesised in order to explain a discrepancy between predicted and observed yield stress in metals $[39,40,45]$, they were subsequently experimentally identified in the 1950 s via electron microscopy $[9,29]$. Dislocations are typically described by a curve in the crystal, called the dislocation line, which is where the resulting distortion is most concentrated, and their Burgers vector, which reflects the mismatch in the lattice they induce [30].

Although the discovery of dislocations is now over 80 years distant, the study of these objects remains of significant interest to Materials Scientists and Engineers 
today. In particular, a cubic centimetre of a metallic solid may contain between $10^{5}$ and $10^{9} \mathrm{~m}$ of dislocation lines [33], leading to a dense networked geometry, and inducing complex stress fields in the material which are relatively poorly understood. Accurately modelling the behaviour of dislocations therefore remains a major hurdle to obtaining predictive models of plasticity on a single crystal scale.

In this work, we propose and study a discrete stochastic model for the thermallydriven motion of interacting straight screw dislocations in a cylindrical crystal of finite diameter. The basic assumptions of this model are that all screw dislocations are aligned with the axis of the cylinder, and that the motion of dislocations proceeds by random jumps between 'adjacent' equilibria, with the rate of jumps being governed by the temperature and the energy barrier between states: this is the minimal additional potential energy which must be gained in order to pass to from one state to another. To describe the system, we prescribe a lattice energy functional, variants of which have been extensively studied in recent literature $[1,2,31,32,41]$.

By rescaling the model in space and time, we identify two dimensionless parameters, and with a specific family of scalings corresponding to a regime in which dislocations are dilute relative to the lattice spacing, the time over which the system is observed is long and the system temperature is low, we find we may apply the theory of Large Deviations described in [21] to obtain a mesoscopic evolution law for the most probable trajectory of a dislocation configuration.

The major novelties of this work are the demonstration of uniqueness (up to symmetries of the model) of equilibria containing dislocations, a precise asymptotic characterisation of the energy barriers between dislocation configurations, and the rigorous identification of both a parameter regime in which the two-dimensional Discrete Dislocation Dynamics framework [3,12,13,46] is valid, as well as a new set of explicit nonlinear anisotropic mobilities which depend upon the underlying lattice structure. The nonlinearity and anisotropy of the mobilities obtained is in contrast to the linear isotropic mobility often assumed in Discrete Dislocation Dynamics simulations.

\subsection{Kinetic Monte Carlo Models}

The stochastic model we formulate is based on the observation that at low temperatures, thermally-driven particle systems spend long periods of time close to local equilibria, or metastable states, before transitioning to adjacent states, and repeating the same process. It is a classical assertion that such transitions are approximately exponentially distributed at low temperatures, with a rate which depends upon the temperature and energy barrier which must be overcome to pass into a new state; the transition rate from state $\mu$ to state $\nu, \mathcal{R}(\mu \rightarrow \nu)$, is given approximately by the formula

$$
\mathcal{R}(\mu \rightarrow v)=\mathcal{A}(\mu \rightarrow v) \mathrm{e}^{-\beta \mathcal{B}(\mu \rightarrow v)}
$$

where

- $\beta:=\left(k_{B} T\right)^{-1}$ is the inverse of the thermodynamic temperature of the system, with $k_{B}$ being Boltzmann's constant and $T$ being the absolute temperature; 
- $\mathcal{B}(\mu \rightarrow \nu)$ is the energy barrier, that is, the additional potential energy relative to the energy at state $\mu$ that the system must acquire in order to pass to the state $v$; and

- $\mathcal{A}(\mu \rightarrow \nu)$ is the entropic prefactor which is related to the 'width' of the pathway by which the system may pass from the state $\mu$ to the state $v$ with minimal potential energy.

The discovery and refinement of the rate formula (1.1) is ascribed to Arrhenius [6], Eyring [20], and Kramers [34], and a review of the physics literature on this subject may be found in [27]. For Itô SDEs with small noise (the usual mathematical interpretation of the correct low-temperature dynamics of a particle system) (1.1) has recently been rigorously validated in the mathematical literature: for a review of recent progress on this subject, we refer the reader to [7].

We may use the observation above to generate a simple coarse-grained model for the thermally-driven evolution of a particle system. Begin by labelling the local equilibria of the system, $\mu$, and prescribe a set of neighbouring equilibria $\mathcal{N}_{\mu}$ which may be accessed from $\mu$, along with the transition rates $\mathcal{R}(\mu \rightarrow \nu)$, for $\nu \in \mathcal{N}_{\mu}$. Given that the system is in a state $\mu$ at time 0 , we model a transition from $\mu$ to a new state $\nu^{\prime} \in \mathcal{N}_{\mu}$ as a jump at a random time $\tau$, where

$$
\begin{gathered}
\tau \sim \min _{v \in \mathcal{N}_{\mu}} \operatorname{Exp}(\mathcal{R}(\mu \rightarrow v))=\operatorname{Exp}\left(\sum_{v \in \mathcal{N}_{\mu}} \mathcal{R}(\mu \rightarrow v)\right) \\
\text { and } \mathbb{P}\left[\mu \rightarrow v^{\prime} \mid \tau=t\right]=\frac{\mathcal{R}\left(\mu \rightarrow v^{\prime}\right)}{\sum_{v \in \mathcal{N}_{\mu}} \mathcal{R}(\mu \rightarrow v)} .
\end{gathered}
$$

This defines a Markov jump process on the set of all states: such processes are sometimes called Kinetic Monte Carlo (KMC) models, and are highly computationally efficient for certain problems in Materials Science [47]. As an example of their use, KMC models have recently been particularly successful in the study of pattern formation during epitaxial growth $[8,44]$. Due to the ease with which samples from exponential random variables may be computed, KMC models allow attainment of significantly longer timescales than Molecular Dynamics simulations of a particle system, with the tradeoff being that fine detail on the precise mechanisms by which phenomena occur may be lost.

A major hurdle in the prescription of a computational KMC model is the definition of the rates $\mathscr{R}(\mu \rightarrow \nu)$. In practice, these must be derived or pre-computed by some means, normally via a costly ab initio or Molecular Dynamics computation run on the underlying particle system to be approximated. Likewise, a large part of the analysis we undertake here is devoted to rigorously deriving an asymptotic expression for energy barrier $\mathcal{B}(\mu \rightarrow \nu)$, which then informs our choice of $\mathcal{R}(\mu \rightarrow \nu)$ using formula (1.1).

\subsection{Modeling Screw Dislocations}

In order to use the KMC framework described above to model the motion of dislocations, we must give an energetic description of the system which allows us to 
define both corresponding metastable states $\mu$ and the energy barriers $\mathcal{B}(\mu \rightarrow \nu)$. In several recent works $[1,2,31,32,41]$, variants of an anti-plane lattice model have been studied in which the notion of the energy of a configuration of straight screw dislocations can be made mathematically precise, and in which screw dislocations may be identified using the topological framework described in [4]. Here, we will follow [31,32] in considering the energy difference

$$
E_{n}(y ; \tilde{y}):=\sum_{e \in \mathrm{D}_{n, 1}}[\psi(\mathbf{d} y(e))-\psi(\mathbf{d} \tilde{y}(e))]
$$

which compares the energy of deformations $y$ and $\tilde{y}$ of a long cylindrical crystal with cross-section $n \mathrm{D}$ : the scaled cross-section $n \mathrm{D} \subset \mathbb{R}^{2}$ is a convex lattice polygon in either the square, triangular or hexagonal lattice, $\mathrm{D}_{n, 1}$ denotes a set of pairs of interacting columns of atoms, $\mathbf{d}$ is a finite difference operator, $y$ and $\tilde{y}$ are antiplane displacement fields, and $\psi$ is a periodic inter-column interaction potential, here taken to be $\psi(s):=\frac{1}{2} \lambda \operatorname{dist}(s, \mathbb{Z})^{2}$.

We define a locally stable equilibrium to be a displacement $y$ such that $u=0$ minimises $E_{n}(y+u ; y)$ among all perturbations which are sufficiently small in the energy norm

$$
\|u\|_{1,2}:=\left(\sum_{e \in \mathrm{D}_{n, 1}}|\mathbf{d} u(e)|^{2}\right)^{1 / 2} .
$$

Configurations containing dislocations are identified by considering bond-length 1-forms associated with $\mathbf{d} y$, the definition of which is recalled in Section 2.5. In analogy with the procedure described in Section 1.3 of [30], this construction allows us to define the Burgers vector in a region of the crystal subject to the deformation $y$ as the integral of the bond-length 1-form around the boundary of the region. This defines a field $\mu$, which we call the dislocation configuration, and we say that the displacement field $y$ contains the dislocations $\mu$.

The results of $[1,2,31]$ demonstrate that there are a large number of locally stable equilibria in this model which contain dislocations for a range of underlying lattice structures. Nevertheless, since these existence results are ultimately all based upon compactness methods, they do not provide a fine description of the equilibria, nor a guarantee of uniqueness up to lattice symmetries, for a fixed choice of the dislocation configuration $\mu$. The first achievement of this work is therefore Theorem 3.1, which provides a novel construction of the equilibria corresponding to dislocation configurations in the particular case where $\psi(s):=\frac{1}{2} \lambda \operatorname{dist}(s, \mathbb{Z})^{2}$. This construction uses a form of lattice duality to show that these minima may be characterised as the 'discrete harmonic conjugate' (interpreted in an appropriate sense) of lattice Green's functions satisfying Dirichlet boundary conditions on a finite lattice domain. In particular, this representation enables us to show that, given a dislocation configuration, there exist corresponding equilibria which are unique up to lattice symmetries. 


\subsection{Energy Barriers}

For two dislocation configurations $\mu$ and $\nu$, we define the energy barrier for the transition from $\mu$ to $v$ as

$$
\mathcal{B}_{n}(\mu \rightarrow v):=\min _{\gamma \in \Gamma_{n}(\mu \rightarrow v)} \max _{t \in[0,1]} E_{n}\left(\gamma(t) ; u_{\mu}\right),
$$

where $u_{\mu}, u_{v}$ are locally stable equilibria containing dislocation configurations $\mu$ and $v$ respectively, and $\Gamma_{n}(\mu \rightarrow v)$ is the space of continuous paths connecting these equilibria. The second major achievement of this work is Theorem 3.2, which gives a precise asymptotic formula for $\mathcal{B}_{n}(\mu \rightarrow \nu)$ as the domain and dislocation configuration are scaled, in terms of the gradient of the renormalised energy $[2,14$, 43]. In the course of proving this result, in Section 5 we constructively demonstrate the existence of transition states $u_{\uparrow}$, such that

$$
E_{n}\left(u_{\uparrow} ; u_{\mu}\right)=\mathcal{B}_{n}(\mu \rightarrow v) .
$$

The construction of $u_{\uparrow}$ again uses the form of lattice duality we describe and lattice Green's functions on the finite domain. Moreover, the properties of Green's functions allow us to compute $\mathcal{B}_{n}(\mu \rightarrow \nu)$ explicitly in terms of a single finite difference of the dual lattice Green's function. In Theorem 4.6, we obtain a precise asymptotic description of this finite difference in terms of the gradient of the continuum renormalised energy as the domain is rescaled, and hence provide an asymptotic formula for $\mathcal{B}_{n}(\mu \rightarrow v)$. Our strategy for proving Theorem 4.6 is to develop a theory akin to the classical gradient estimates for solutions of Poisson's equation (see Section 3.4 of [24]) in a discrete setting.

\subsection{Upscaling via a Large Deviations Principle}

Once we have obtained the asymptotic representation of $\mathcal{B}_{n}(\mu \rightarrow v)$ given in Theorem 3.2, we apply formula (1.1) to define the rates $\mathcal{R}_{n}(\mu \rightarrow \nu)$ and hence the stochastic model considered. We then seek to understand the behaviour of this model in the regime where the distance between dislocations is significantly larger than the lattice spacing. Scaling the various physical constants inherent in the model enables us to identify two non-dimensional constants which govern the evolution.

Fixing these constants leads us to consider the asymptotic regime in which the temperature is low, the diameter of the cylindrical domain and the spacing between dislocations is large relative with the lattice spacing, and the time over which the process is observed is long. In this regime, we find that the processes satisfy a Large Deviations Principle, which provides a means of describing the asymptotic probability of rare events in random processes. A general theoretical framework for proving such results has been developed over the last 50 years, and major treatises on the subject describing a variety of approaches include [16,18,21,22].

More precisely, a sequence of random variables $X^{n}$ taking values on a metric space $M$ is said to satisfy a Large Deviations Principle if there exists a lower semicontinuous functional $\mathcal{I}: M \rightarrow[0,+\infty]$ such that for any open set $A \subseteq M$,

$$
\liminf _{n \rightarrow \infty} \frac{1}{n} \log \mathbb{P}\left[X^{n} \in A\right] \geqq-\inf _{x \in A} \mathcal{I}(x),
$$


and for any closed set $B \subseteq M$, we have that

$$
\limsup _{n \rightarrow \infty} \frac{1}{n} \log \mathbb{P}\left[X^{n} \in B\right] \leqq-\inf _{x \in B} \mathcal{I}(x) .
$$

The function $\mathcal{I}$ is called the rate function of the Large Deviations Principle, and is called good if each of the sub-level sets $\{x \mid \mathcal{I}(x) \leqq a\}$ for $a \in \mathbb{R}$ is compact in $M$ (a property normally referred to as coercivity in the Calculus of Variations literature). The existence of a Large Deviations Principle may be interpreted as saying that, for any Borel set $A$,

$$
\mathbb{P}\left[X^{n} \in A\right] \simeq \exp \left(-n \inf _{x \in A} \mathcal{I}(x)\right), \quad \text { as } n \rightarrow \infty,
$$

i.e. the probability of observing events disjoint from $\mathcal{I}^{-1}(0)$ becomes exponentially small as $n \rightarrow \infty$.

In the setting considered here, the random variables $X^{n}$ correspond to trajectories of the dislocation configuration through an appropriate state space. In order to prove a Large Deviations Principle, we apply the theory developed in [21] and summarise the main results of this treatise in a form suited to our application in Theorem 3.3. The existence of a Large Deviations Principle is then asserted in Theorem 3.4, which also gives an explicit description of the 'most probable' trajectory of the system. This trajectory corresponds to a solution of the equations usually simulated in the study of Discrete Dislocation Dynamics [3,12,13], with an explicit anisotropic mobility function $\mathcal{M}_{A, B}^{\mathrm{L}}$ which depends upon the underlying lattice structure.

We conclude our study by discussing the interpretation of this result, and show that the additional regimes identified in [10] also apply here: in particular, we show it is possible to recover the linear gradient flow structure normally used in Discrete Dislocation Dynamics simulations $[3,12,13]$ in a further parametric limit, but we argue that in the appropriate parameter regimes, a stochastic evolution problem may be more appropriate to model dislocation evolution.

\subsection{Structure and Notation}

In order to give a precise statement of our main results, Section 2 is devoted to describing the geometric framework which is both used to describe the Burgers vector of a lattice deformation in our model and the notion of duality which we use in the subsequent analysis.

In Section 3, we state and discuss our main results. These are Theorem 3.1, which characterises equilibria containing dislocations, Theorem 3.2, which provides a precise asymptotic formula for the energy barrier between equilibria, and Theorem 3.4, which asserts the existence of a Large Deviations Principle for the Markov processes and asymptotic regime we consider. The proofs of these results are given in Sects. 4, 5 and 6 respectively.

Since we introduce significant amounts of notation in order to concisely state our results, Table 1 is provided for convenience. 
Table 1. Notation conventions

\begin{tabular}{|c|c|}
\hline Symbol & Description \\
\hline L & $m$-dimensional multilattice identified with a lattice complex \\
\hline $\operatorname{Tr}, \mathrm{Sq}, \mathrm{Hx}$ & Triangular, square and hexagonal lattices in $\mathbb{R}^{2}$ \\
\hline $\mathcal{K}, \mathcal{K}^{*}, \mathcal{V}, \mathcal{V}^{*}$ & Constants depending on $\mathrm{L}$ \\
\hline D & Convex lattice polygon \\
\hline$c_{l}, \varphi_{l}$ & Position and interior angle of corner $l$ of $\mathrm{D}$ \\
\hline $\mathrm{L}_{p}, \mathrm{~L}_{p}^{*}$ & Set of $p$-cells in the primal and dual lattice complexes induced by $\mathrm{L}$ \\
\hline $\mathrm{D}_{n, p}, \mathrm{D}_{n, p}^{*}$ & $\begin{array}{l}\text { Set of } p \text {-cells in the primal and dual lattice subcomplexes } \\
\text { induced by } n \mathrm{D}\end{array}$ \\
\hline $\operatorname{Ext}\left(\mathrm{D}_{n, p}\right)$ & Set of $p$-cells in $\mathrm{D}_{n, p}$ at the 'edge' of the complex induced by $n \mathrm{D}$ \\
\hline $\operatorname{Int}\left(\mathrm{D}_{n, p}\right)$ & Set of $p$-cells in $\mathrm{D}_{n, p}$ which lie 'away from the edge of $n \mathrm{D}^{\prime}$ \\
\hline$e$ & A $p$-cell \\
\hline$\left[e_{0}, e_{1}\right]$ & 1-cell $e$ such that $\partial e=e_{1} \cup-e_{0}$. \\
\hline$e+\mathrm{a}$ & $p$-cell obtained by translating $e$ by the vector a \\
\hline$\partial, \delta$ & Boundary and coboundary operators \\
\hline d, $\delta$ & $\begin{array}{l}\text { Differential and codifferential on forms defined on the lattice } \\
\text { complex }\end{array}$ \\
\hline$\Delta$ & Hodge Laplacian on forms \\
\hline $\mathscr{W}\left(\mathrm{L}_{p}\right), \mathscr{W}\left(\mathrm{D}_{n, p}\right)$ & Set of $p$-forms on $L$ and $D$ \\
\hline $\mathscr{L}^{2}\left(\mathrm{~L}_{p}\right)$ & Hilbert space of square-integrable $p$-forms on $\mathrm{L}$ \\
\hline $\mathscr{W}_{0}\left(\mathrm{D}_{n, p}\right)$ & Set of $p$-forms on $\mathrm{D}$ which vanish on $\operatorname{Ext}\left(\mathrm{D}_{n, p}\right)$ \\
\hline$(\cdot)^{*}(n, P)$ & Duality mapping on $p$-cells and $p$-forms \\
\hline$E_{n}(y ; \tilde{y})$ & Energy difference between deformations $y$ and $\tilde{y}$ \\
\hline$\psi$ & $\begin{array}{l}\text { Potential giving energy per unit length of interaction between } \\
\text { columns of atoms }\end{array}$ \\
\hline$[\mathbf{d} u]$ & Set of bond-length 1 -forms corresponding to $\mathbf{d} u$ \\
\hline$u_{\mu}$ & Locally stable equilibrium containing dislocations $\mu$ \\
\hline $\mathcal{R}_{n}(\mu \rightarrow v)$ & Exponential transition rate to pass from $\mu$ to $v$ \\
\hline $\mathcal{A}_{n}(\mu \rightarrow \nu)$ & Entropic prefactor for transition from $\mu$ to $v$ \\
\hline $\mathcal{B}_{n}(\mu \rightarrow v)$ & Potential energy barrier to transition from $\mu$ to $v$ \\
\hline$\Gamma_{n}(\mu \rightarrow v)$ & Space of paths in deformation space connecting $u_{\mu}$ and $u_{\nu}$ \\
\hline$u_{\uparrow}$ & Transition state, i.e. deformation where $\mathcal{B}_{n}(\mu \rightarrow v)$ is attained \\
\hline $\begin{array}{l}\alpha_{\uparrow}, \alpha_{\downarrow} \\
\mathbb{1}_{e}\end{array}$ & $\begin{array}{l}\text { Bond-length } 1 \text {-forms corresponding to the transition state } \\
p \text {-form which is } \pm 1 \text { on } \pm e \text { and } 0 \text { otherwise }\end{array}$ \\
\hline$G^{\mathrm{L}}$ & Green's function for the full lattice $L$ \\
\hline$Q^{r}$ & Polygonal set of radius $r$ in the lattice \\
\hline$\omega_{e}^{r}$ & Harmonic measure for $Q^{r}$ evaluated at $e \in \operatorname{Ext}\left(Q_{0}^{r}\right)$ \\
\hline$G_{\mu^{*}}$ & Solution to $\Delta^{*} G_{\mu^{*}}=\mu^{*}$ in $\mathscr{W}_{0}\left(D_{n, 0}^{*}\right)$ \\
\hline $\mathcal{G}_{y}$ & $\begin{array}{l}\text { Continuum Green's function, solving }-\Delta \mathcal{G}_{y}=\frac{1}{\mathcal{V}} \delta_{y} \text { in } \mathrm{D}, \mathcal{G}_{y}= \\
0 \text { on } \partial \mathrm{D}\end{array}$ \\
\hline $\mathscr{M}_{n}^{\varepsilon}$ & Set of 'well-separated' dislocation positions \\
\hline $\mathscr{M}_{\infty}^{\mathcal{\varepsilon}}$ & Set of macroscale 'well-separated' dislocation positions \\
\hline$\beta$ & Inverse thermodynamic temperature \\
\hline $\mathcal{T}_{n}$ & Characteristic timescale of observation \\
\hline $\mathrm{D}([0, T] ; M)$ & $\begin{array}{l}\text { Skorokhod space of càdlàg functions from }[0, T] \\
\text { to a metric space } M\end{array}$ \\
\hline$\Omega_{n}, H_{n}$ & Infinitesimal and nonlinear generators of the KMC process \\
\hline $\mathcal{H}_{A, B}^{\mathrm{L}}, \mathcal{L}_{A, B}^{\mathrm{L}}$ & The Hamiltonian and Lagrangian for KMC process \\
\hline $\mathcal{E}^{R, D}$ & Renormalised energy \\
\hline & Dissipation potential \\
\hline $\mathcal{J}_{A, B}^{\mathrm{L}}$ & Large Deviations rate functional \\
\hline
\end{tabular}




\section{Preliminaries}

As stated in the introduction, the construction of the local minima corresponding to dislocation configurations we give below relies upon a particular dual construction which corresponds in some sense to the construction of a 'discrete harmonic conjugate'. This construction is most conveniently expressed using a discrete theory of differential forms, which also provides the basis for a definition of the Burgers vector of a deformation. The reader already familiar with this theory may wish to refer to Table 1 for our choice of notation and skip to Section 2.3, where the particular examples necessary for the subsequent analysis are given.

\subsection{Lattice Complex}

We begin by recalling some facts about lattice complexes, which provide the correct tools to study dislocations in the model we consider. Lattice complexes are a particular class of $C W$ complex, which are objects usually studied in algebraic topology, and were defined with a particular view to applications in the modelling of dislocations in crystals in [4]: we follow the same basic definitions and terminology here. For further details on the definitions below, we refer the reader to Section 2 of [4], and for background on such constructions in a general setting, see either the Appendix of [28], or [38].

To provide some intuition to those less familiar with the notions described here, we remark at the outset that a lattice complex may be thought of as a 'skeleton' of sets of increasing dimension which is built on the lattice points and fills $\mathbb{R}^{d}$. The elements of this skeleton are $p$-cells, where $p$ refers to the 'dimension' of the particular element. The key idea behind the definition of a lattice complex is that it provides a means by which to make rigorous sense of

- the boundaries of sets;

- operators analogous to the gradient, divergence, and curl, and

- versions of the Divergence and Stokes' theorems which relate the above notions.

Since these are likely to be familiar, we will point out some analogies with these more familiar calculus concepts along the way. The reader is invited to refer to Fig. 1 for an illustration of the particular lattice complexes used in the subsequent analysis.

2.1.1. Construction of a Lattice Complex Given a Hausdorff topological space $S$, a 0 -cell is simply a member of some fixed subset of points in $S$. Higherdimensional cells are then defined iteratively: for $p \geqq 1$, a $p$-dimensional cell (or $p$-cell) is $e \subset S$ for which there exists a homeomorphism mapping the interior of the $p$-dimensional closed ball in $\mathbb{R}^{p}$ onto $e$, and mapping the boundary of the ball onto a finite union of cells of dimension less than $p$.

A CW complex is a Hausdorff topological space along with a collection of cells as defined above, such that $S$ is the disjoint union of all cells. The CW complex is $d$-dimensional if the maximum dimension of any cell is $d$, and $S$ is referred to as the underlying space of the complex: $S_{p}$ will denote the set of all $p$-cells in the complex. 

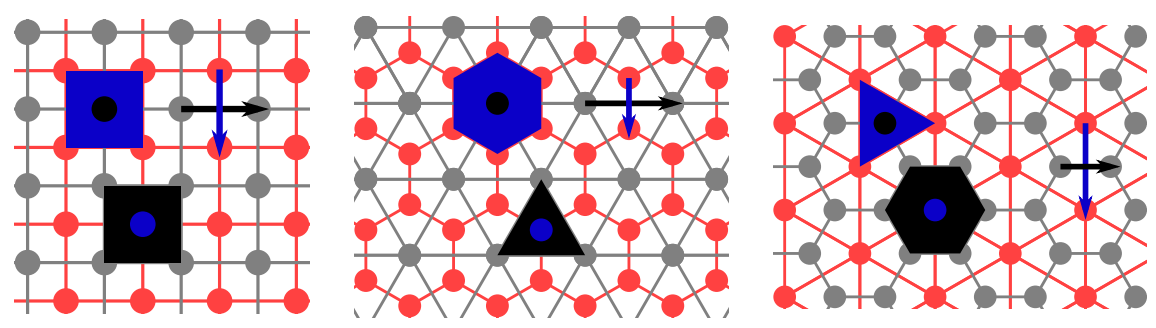

Fig. 1. The square, triangular and hexagonal lattices respectively, and their duals. Primal lattices are shown in grey, dual lattices in red; 2-cells are left uncoloured. Particular primal cells are highlighted in black, and their respective dual cells are given in blue (colour figure online)

Each $p$-cell may be assigned an orientation consistent with the usual notion for set in $\mathbb{R}^{d}$, and we write $-e$ to mean the $p$-cell with opposite orientation to that of $e$. We may define an operator $\partial$, called the boundary operator, which maps oriented $p$-cells to consistently oriented $(p-1)$-cells, which intuitively are 'the boundary' of the original cell. Similarly, the coboundary operator $\delta$ may be defined, mapping an oriented $p$-cell, $e$, to all consistently oriented $p+1$-cells which have $e$ as part of their boundary.

We now recall from [4] that a lattice complex is a $\mathrm{CW}$ complex such that:

- the underlying space is all of $\mathbb{R}^{d}$,

- the set of 0-cells forms an $d$-dimensional lattice, and

- the cell set is translation and symmetry invariant.

Throughout, we will denote such a lattice complex $L$, and the set of $p$-cells of the corresponding complex will be $\mathrm{L}_{p}$. Due to the translation invariance of $\mathrm{L}$, it will be particularly convenient to consider translations of lattice $p$-cells, so for $e \in \mathrm{L}_{p}$ and a vector $\mathrm{a} \in \mathbb{R}^{d}$, we define

$$
e+\mathrm{a}:=\left\{x \in \mathbb{R}^{d} \mid x=y+\mathrm{a}, y \in e\right\} .
$$

We will always assume that we have chosen coordinates such that $\{0\} \in \mathrm{L}_{0}$ and, abusing notation, we will write 0 to refer to this 0 -cell.

A second convenient notational convention we will occasionally use is the representation of a 1-cell through its boundary; we write

$$
e=\left[e_{0}, e_{1}\right] \text { to mean } e \in \mathrm{L}_{1} \text { such that } \partial e=e_{1} \cup-e_{0} \text {. }
$$

2.1.2. Spaces of $p$-Forms and Calculus on Lattices For the application considered here, we wish to describe deformations of a crystal. These are appropriately described in the lattice complex framework as $p$-forms, which are real-valued functions on $p$-cells which change sign if the orientation of the cell on which they are evaluated is reversed. We define $\mathscr{W}\left(\mathrm{L}_{p}\right)$ to be the space of all $p$-forms, that is

$$
\mathscr{W}\left(\mathrm{L}_{p}\right):=\left\{f: \mathrm{L}_{p} \rightarrow \mathbb{R} \mid f(e)=-f(-e), \text { for any } e \in \mathrm{L}_{p}\right\} .
$$


It is straightforward to check that this is a vector space under pointwise addition. We also define the set of compactly-supported $p$-forms,

$$
\mathscr{W}_{c}\left(\mathrm{~L}_{p}\right):=\left\{f \in \mathscr{W}\left(\mathrm{L}_{p}\right) \mid \overline{\bigcup\{e \mid f(e) \neq 0\}} \text { is compact in } \mathbb{R}^{d}\right\}
$$

where here and throughout, $\bar{A}$ denotes the closure of $A \subset \mathbb{R}^{d}$.

Let $A \subset \mathrm{L}_{p}$ be finite; then for $f \in \mathscr{W}\left(\mathrm{L}_{p}\right)$, we define the integral

$$
\int_{A} f:=\sum_{e \in A} f(e) .
$$

The differential and codifferential are respectively the linear operators $\mathbf{d}: \mathscr{W}\left(\mathrm{L}_{p}\right) \rightarrow$ $\mathscr{W}\left(\mathrm{L}_{p-1}\right)$ and $\delta: \mathscr{W}\left(\mathrm{L}_{p}\right) \rightarrow \mathscr{W}\left(\mathrm{L}_{p+1}\right)$, defined to be

$$
\mathbf{d} f(e):=\int_{\partial e} f \quad \text { and } \quad \boldsymbol{\delta} f(e):=\int_{\delta e} f .
$$

For a 0 -form on a lattice complex, the differential is simply the finite difference operator defined for a pair of nearest neighbours, and in a continuous setting the same operator is the gradient. Similary, $\boldsymbol{\delta}$ acting on 1 -forms is either (the negative of) the discrete or continuum divergence operator. In a three-dimensional complex, both $\mathbf{d}$ acting on 1 -forms and $\boldsymbol{\delta}$ acting on 2-forms may be thought of as the curl operator.

The bilinear form

$$
(f, g):=\int_{\mathrm{L}_{p}} f g
$$

is well-defined whenever $f \in \mathscr{W}_{c}\left(\mathrm{~L}_{p}\right)$ or $g \in \mathscr{W}_{c}\left(\mathrm{~L}_{p}\right)$. Moreover, if $f \in \mathscr{W}_{c}\left(\mathrm{~L}_{p}\right)$ and $g \in \mathscr{W}_{c}\left(\mathrm{~L}_{p+1}\right)$, we have the integration by parts formula

$$
(\mathbf{d} f, g)=(f, \boldsymbol{\delta} g)
$$

this statement should be compared with that of the Divergence Theorem and variants, using the vector calculus interpretation of $\mathbf{d}$ and $\boldsymbol{\delta}$ given above. Furthermore, by defining the space $\mathscr{L}^{2}\left(\mathrm{~L}_{p}\right):=\left\{f \in \mathscr{W}\left(\mathrm{L}_{p}\right) \mid(f, f)<+\infty\right\}$, this bilinear form defines an inner product. It is straightforward to show that this is then a Hilbert space with the induced norm, which we denote $\|u\|_{2}:=(u, u)^{1 / 2}$.

We recall the definition of the Hodge Laplacian as the operator

$$
\boldsymbol{\Delta}: \mathscr{W}\left(\mathrm{L}_{p}\right) \rightarrow \mathscr{W}\left(\mathrm{L}_{p}\right) \text { with } \quad \boldsymbol{\Delta} f:=(\boldsymbol{\delta} \mathbf{d}+\mathbf{d} \boldsymbol{\delta}) f
$$

when $p \neq 0$ and $p \neq m$, and in the cases where $p=0$ and $p=m, \boldsymbol{\Delta}=\delta \mathbf{d}$ and $\boldsymbol{\Delta}=\mathbf{d} \boldsymbol{\delta}$ respectively. Note that, in a continuum setting, this definition of the Laplacian agrees with the interpretation of $\mathbf{d}$ as the gradient on 0 -forms and $\delta$ as the negative of the divergence on 1-forms. Any function satisfying $\Delta f=0$ on $A \subset \mathrm{L}_{p}$ is said to be harmonic on $A$.

Finally, $\mathbb{1}_{e}$ will always denote the $p$-form

$$
\mathbb{1}_{e}\left(e^{\prime}\right):=\left\{\begin{aligned}
\pm 1 & e^{\prime}= \pm e \\
0 & \text { otherwise }
\end{aligned}\right.
$$




\subsection{Dual Complex}

The common notion of duality which occurs in algebraic topology relating to $\mathrm{CW}$ complexes is that of the cohomology. This is usually presented as an abstract algebraic structure, since it is only this structure which is needed to deduce topological information about a CW complex. In some cases it may also be given a more concrete identification, which will be particularly important for the subsequent analysis.

Given an $m$-dimensional lattice complex, when possible, we define the dual complex as follows:

- For any $e \in \mathrm{L}_{m}$, let $e^{*}:=\int_{e} x \mathrm{~d} x$, the barycentre of set $e$ in $\mathbb{R}^{d}$, and let

$$
\mathrm{L}_{0}^{*}:=\left\{e^{*} \mid e \in \mathrm{L}_{m}\right\} .
$$

- For a collection of elementary $m$-cells $A \in \mathrm{L}_{m}$, let

$$
A^{*}:=\bigcup_{e \in A} e^{*}
$$

- Now, iterate over $p=m-1, m-2, \ldots, 0$ : for each $p$, let $e \in \mathrm{L}_{p}$, and consider $\delta e \in \mathrm{L}_{p+1}$ as a sum of elementary $p$-cells. Find the corresponding cells in $\mathrm{L}_{m-p-1}^{*}$. Define $e^{*} \in \mathrm{L}_{m-p}^{*}$ to be the convex hull of $(\delta e)^{*}$ with $(\delta e)^{*}$ removed, assigning $e^{*}$ the same orientation as $e$. For $A$, a sum of elementary $p$-cells, we again define $A^{*}$ via (2.3).

We define boundary and coboundary operators on the dual lattice complex, $\partial^{*}$ and $\delta^{*}$, so that

$$
\partial^{*} e^{*}=(\delta e)^{*}, \text { and } \delta^{*} e^{*}=(\partial e)^{*} .
$$

By construction, $*: L_{p} \rightarrow L_{m-p}^{*}$ defines an isomorphism of the additive group structure usually defined on lattice complexes (see Section 2.2 of [4]). The equalities stated in (2.4) may then be interpreted as the statement of the Poincaré duality theorem (see for example Section 3.3 of [28]), and the construction described above is succinctly represented in the following commutation diagram.



Since the differential and codifferential operators inherit features from the structure of the CW complex on which $p$-forms are defined, we now show that similar duality properties hold for the differential complexes on $L$ and $L^{*}$. For any $f \in \mathscr{W}\left(\mathrm{L}_{p}\right)$, we define $f^{*} \in \mathscr{W}\left(\mathrm{L}_{m-p}^{*}\right)$ via

$$
f^{*}\left(e^{*}\right):=f(e)
$$


Again, it may be checked that $*: \mathscr{W}\left(\mathrm{L}_{p}\right) \rightarrow \mathscr{W}\left(\mathrm{L}_{m-p}^{*}\right)$ is an isomorphism; in fact, $*$ defines an isometry of the spaces $\mathscr{L}^{2}\left(\mathrm{~L}_{p}\right)$ and $\mathscr{L}^{2}\left(\mathrm{~L}_{m-p}^{*}\right)$. The differential, denoted $\mathbf{d}^{*}: \mathscr{W}\left(\mathrm{L}_{p}^{*}\right) \rightarrow \mathscr{W}\left(\mathrm{L}_{p-1}^{*}\right)$, and codifferential, denoted $\delta^{*}: \mathscr{W}\left(\mathrm{L}_{p-1}^{*}\right) \rightarrow \mathscr{W}\left(\mathrm{L}_{p}^{*}\right)$, are then

$$
\begin{aligned}
\mathbf{d}^{*} f^{*}\left(e^{*}\right) & :=\int_{\partial^{*} e^{*}} f^{*}=\int_{\delta e} f=\boldsymbol{\delta} f(e), \\
\text { and } \delta^{*} f^{*}\left(e^{*}\right) & :=\int_{\delta^{*} e^{*}} f^{*}=\int_{\partial e} f=\mathbf{d} f(e) .
\end{aligned}
$$

Again, this relationship is concisely expressed in the following diagram.

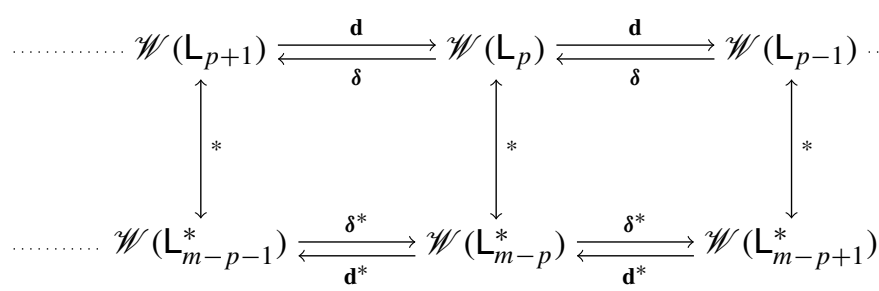

\subsection{Examples: The Square, Triangular and Hexagonal Lattices}

In the analysis which follows, we focus exclusively on 2-dimensional lattice complexes, and in particular the triangular, square and hexagonal lattices denoted $\mathrm{Tr}, \mathrm{Sq}$ and $\mathrm{Hx}$ respectively. Let $\mathrm{R}_{4}$ and $\mathrm{R}_{6}$ be the rotation matrices

$$
\mathrm{R}_{4}:=\left(\begin{array}{cc}
0 & -1 \\
1 & 0
\end{array}\right) \quad \text { and } \quad \mathrm{R}_{6}:=\left(\begin{array}{cc}
\frac{1}{2} & -\frac{\sqrt{3}}{2} \\
\frac{\sqrt{3}}{2} & \frac{1}{2}
\end{array}\right)
$$

For convenience, we define $e_{1}:=a_{1}:=(1,0)^{T}$, and

$$
\mathrm{e}_{i}:=\mathrm{R}_{4}^{i-1} \mathrm{e}_{1} \text { for } i \in\{1,2,3,4\}, \text { and } \mathrm{a}_{j}:=\mathrm{R}_{6}^{j-1} \mathrm{a}_{1} \text { for } j \in\{1, \ldots, 6\} .
$$

The triangular, square and hexagonal lattices are defined to be

$$
\operatorname{Tr}:=\left[\mathrm{a}_{1}, \mathrm{a}_{2}\right] \cdot \mathbb{Z}^{2}, \quad \mathrm{Sq}:=\mathbb{Z}^{2}, \quad \text { and } \mathrm{Hx}:=\sqrt{3} \mathrm{R}_{4} \operatorname{Tr} \cup\left[\sqrt{3} \mathrm{R}_{4} \operatorname{Tr}+\mathrm{e}_{1}\right] ;
$$

the nearest neighbour directions in $\mathrm{Sq}$ are therefore $\mathrm{e}_{i}$, and $\mathrm{a}_{i}$ in $\mathrm{Tr}$ or $\mathrm{Hx}$. We may define lattice complexes based on these sets (see Sects. 2.3.2 and 2.3.3 of [4] and [5]), and moreover

$$
\begin{aligned}
\operatorname{Tr}^{*} & =\frac{\sqrt{3}}{3} \mathrm{R}_{4} \mathrm{Hx}+\frac{1}{3}\left(\mathrm{a}_{2}+\mathrm{a}_{3}\right), \\
\mathrm{Sq}^{*} & =\mathrm{Sq}+\frac{1}{2}\left(\mathrm{e}_{1}+\mathrm{e}_{2}\right), \\
\text { and } \mathrm{Hx}^{*} & =\sqrt{3} \mathrm{R}_{4} \mathrm{Tr}+\frac{\sqrt{3}}{3}\left(\mathrm{a}_{1}+\mathrm{a}_{2}\right) .
\end{aligned}
$$

Figure 1 illustrates the three lattices and the duality mapping between $L$ and $L^{*}$. 

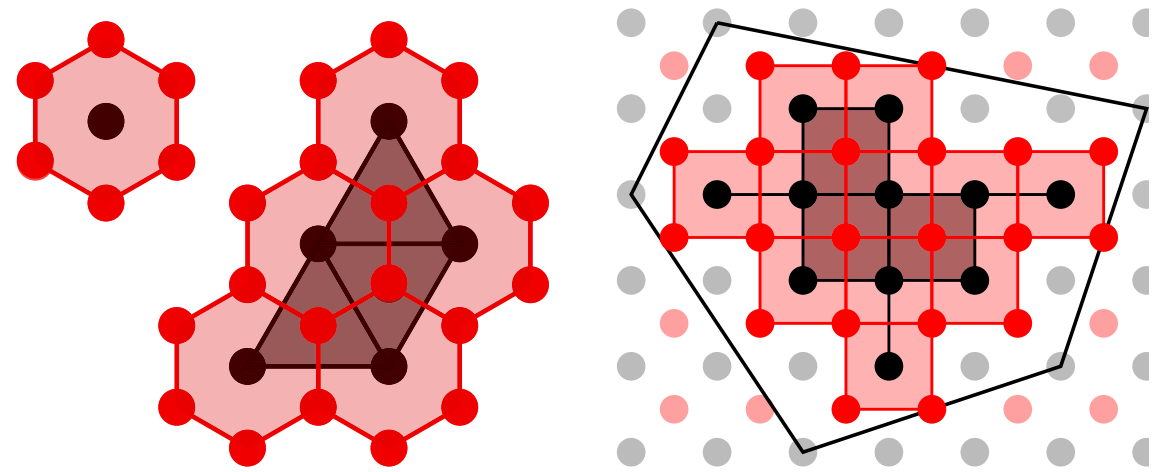

Fig. 2. On the left, an example of primal (in black) and dual (in red) induced subcomplexes for a general subset of the triangular lattice: $A_{0}$ is the set of black points. On the right, a lattice polygon $\mathrm{D}$ in the square lattice, and the corresponding primal and dual subcomplexes, which are both path- and simply-connected (colour figure online)

At this point, we give the definitions of some lattice-dependent constants which will arise during our analysis:

$$
\mathcal{K}:=\left\{\begin{array}{ll}
3 & \text { if } L=\mathrm{Hx}, \\
4 & \text { if } L=\mathrm{Sq}, \\
6 & \text { if } L=\mathrm{Tr}
\end{array} \quad \text { and } \quad \mathcal{V}:= \begin{cases}2 & \text { if } \mathrm{L}=\mathrm{Hx} \\
4 & \text { if } \mathrm{L}=\mathrm{Sq} \\
6 & \text { if } \mathrm{L}=\mathrm{Tr}\end{cases}\right.
$$

For convenience, we will write $\mathcal{V}^{*}$ and $\mathcal{K}^{*}$ to mean the relevant constants for the dual lattice. Note that $\mathcal{K}$ is the number of nearest neighbours in the lattice.

\subsection{Finite Lattice Subcomplexes}

For the particular application we will consider, we will make use of finite subcomplexes of the full lattice complex, and so we now make precise the notation we use as well as the particular assumptions made throughout our analysis. The reader may find it useful to refer to Fig. 2, which illustrates the construction in a couple of simple cases.

2.4.1. Induced Subcomplexes Given a finite subset $A_{0} \subset L_{0}$, we define the induced lattice subcomplex by inductively defining

$$
\mathrm{A}_{p}:=\left\{e \in \mathrm{L}_{p} \mid \partial e \subset \mathrm{A}_{p-1}\right\}
$$

This is a well-defined CW complex when the corresponding boundary $\partial^{\mathrm{A}}$ and coboundary $\delta^{A}$ operators are defined by restriction, i.e.

$$
\partial^{\mathrm{A}} e:=\partial e \cap \mathrm{A}_{p-1}, \quad \text { and } \quad \delta^{\mathrm{A}} e=(\delta e) \cap \mathrm{A}_{p+1} \text { for all } e \in \mathrm{A}_{p} .
$$

The induced differential and codifferential operators $\mathrm{d}^{\mathrm{A}}$ and $\delta^{\mathrm{A}}$ are then defined in the same way as $\mathbf{d}$ and $\delta$, using $\partial^{\mathrm{A}}$ and $\delta^{\mathrm{A}}$ in place of $\partial$ and $\delta$, and we may define the spaces $\mathscr{W}\left(\mathrm{A}_{p}\right)$ and $\mathscr{L}^{2}\left(\mathrm{~A}_{p}\right)$. 
It will be convenient to distinguish what we term the exterior and interior $p$-cells of the $\mathrm{CW}$ complex $\mathrm{A}$, respectively defined to be

$$
\operatorname{Ext}\left(\mathrm{A}_{p}\right):=\left\{e \in \mathrm{A}_{p} \mid \delta e \neq \delta^{\mathrm{A}} e\right\}, \quad \text { and } \operatorname{Int}\left(\mathrm{A}_{p}\right):=\mathrm{A}_{p} \backslash \operatorname{Ext}\left(\mathrm{A}_{p}\right) .
$$

The former set may be thought of as the 'edge' of the lattice subcomplex, and the latter as the 'interior' of the lattice subcomplex.

We now define a subcomplex of the dual lattice complex which we call the dual subcomplex induced by $\mathrm{A}_{0}$. Let $\mathrm{A}_{m}^{*}:=\left\{e^{*} \in \mathrm{L}_{m}^{*} \mid e \in \mathrm{A}_{0}\right\}$, and inductively define

$$
\mathrm{A}_{m-p}^{*}:=\left\{e^{*} \in \mathrm{L}_{m-p}^{*} \mid e^{*} \in \partial^{*} a^{*} \text { for some } a^{*} \in \mathrm{A}_{m-p+1}^{*}\right\}
$$

for $p \geqq 1$. We remark that this definition is not equivalent to defining sets of sets of dual $p$-cells by directly taking the dual of the primal $p$-cells; however, we do have the inclusion

$$
\left[\mathrm{A}_{p}\right]^{*} \subseteq \mathrm{A}_{m-p}^{*} \text { for each } p,
$$

where equality always holds when $p=m$ by definition. The other inclusions follow by induction on $p$ : note that $e \in \mathrm{A}_{p}$ with $p \geqq 1$ implies that $e \in \delta^{\mathrm{A}} a$ for some $a \in \mathrm{A}_{p-1}, \delta^{\mathrm{A}} a \subseteq \delta a$, and hence $e^{*} \in \partial^{*} a^{*}$ for some $a^{*} \in \mathrm{A}_{m-p+1}^{*}$. As before, we may define $\partial^{A^{*}}$ and $\delta^{A^{*}}$ by restriction, which in turn leads us to define operators $\mathbf{d}^{\mathrm{A}^{*}}$ and $\delta^{\mathrm{A}^{*}}$ analogously.

Similarly, let

$$
\operatorname{Ext}\left(\mathrm{A}_{p}^{*}\right):=\left\{e^{*} \in \mathrm{A}_{p}^{*} \mid \delta^{*} e^{*} \neq \delta^{\mathrm{D}^{*}} e^{*}\right\}, \quad \text { and } \operatorname{Int}\left(\mathrm{A}_{p}^{*}\right):=\mathrm{A}_{p}^{*} \backslash \operatorname{Ext}\left(\mathrm{A}_{p}^{*}\right)
$$

By construction, $\operatorname{Ext}\left(\mathrm{A}_{2}^{*}\right)=\emptyset$, and $e \in \operatorname{Ext}\left(\mathrm{A}_{n-p}^{*}\right)$ if and only if there exists no $a \in \mathrm{A}_{p}$ with $e=a^{*}$ (see Fig. 2 for an illustration).

From now on, it will always be clear from the context whether we are referring to the relevant operators on $L$ and $L^{*}$, or on $A$ and $A^{*}$, so for the sake of concision, we will suppress $A$ from our notation.

2.4.2. Subcomplexes Induced by a Domain We will say that an induced lattice subcomplex is path-connected if for any $e, e^{\prime} \in \mathrm{A}_{0}$, there exists $\gamma \subset \mathrm{A}_{1}$ such that

$$
\partial \gamma=e \cup-e^{\prime}
$$

and call such $\gamma \subset \mathrm{A}_{1}$ a path which connects $e$ and $e^{\prime}$. We will say a lattice subcomplex is simply-connected if for any $\gamma^{\prime} \subset A_{1}$ such that $\partial \gamma=\emptyset, \gamma=\partial A$ for some $A \in \mathrm{A}_{2}$.

Throughout our analysis, D will always denote a closed convex lattice polygon, i.e. a non-empty compact convex subset of $\mathbb{R}^{2}$ which has corners $c_{l} \in \mathrm{L}$ and internal angles $\varphi_{l}$ where $l=1, \ldots, L$ indexes the corners, following [25]. We consider the scaled domains $n \mathrm{D}$, for $n \in \mathbb{N}$, noting that $n \mathrm{D}$ remains a lattice polygon, and denote $\mathrm{D}_{n}$ to be the largest induced lattice subcomplex with respect to inclusion such that

- $\mathrm{D}_{n, p} \subset n \mathrm{D}$ for all $p$,

- $\mathrm{D}_{n, p}^{*} \subset n \mathrm{D}$ for all $p$,

- $\mathrm{D}_{n}$ and $\mathrm{D}_{n}^{*}$ are both path connected and simply connected.

It can be shown that such a complex always exists as long as $n$ is sufficiently large, since $D$ is convex: we give an example on the right-hand side of Fig. 2. 
2.4.3. Counting and Distances For a collection of $p$-cells $A \subset \mathrm{D}_{n, p}$, we write $\# A$ to designate the smallest number of elementary $p$-cells $e_{i}$ such that $A=$ $\bigcup_{i=1}^{\# A} e_{i}$.

We define diam(D) to be

$$
\operatorname{diam}(\mathrm{D}):=\max \{|x-y| \mid x, y \in \mathrm{D}\},
$$

and we note that there exists a constant $C^{\mathrm{L}}>0$ which depends only on the underlying lattice $L$ such that

$$
\max \left\{\min \left\{\# \gamma \mid \gamma \subset \mathrm{D}_{n, 1}, \partial \gamma=e-e^{\prime}\right\} \mid e^{\prime}, e \in \operatorname{Ext}\left(\mathrm{D}_{n, 0}\right)\right\} \leqq C^{\mathrm{L}} n \operatorname{diam}(\mathrm{D}) .
$$

We write $\operatorname{dist}(A, B)$ to mean the shortest distance between two sets $A, B \subset \mathbb{R}^{d}$, i.e.

$$
\operatorname{dist}(A, B):=\inf \{|x-y| \mid x \in A, y \in B\} .
$$

2.4.4. Spaces of $p$-Forms on Lattice Subcomplexes The space of $p$-forms on the lattice subcomplex induced by $n \mathrm{D}$ is denoted

$$
\mathscr{W}\left(\mathrm{D}_{n, p}\right):=\left\{u: \mathrm{D}_{n, p} \rightarrow \mathbb{R} \mid u(e)=-u(-e)\right\} .
$$

As for the space of forms defined on $L$, we define the inner product and induced norm

$$
(u, v):=\int_{\mathrm{D}_{n, p}} u v, \quad \text { and }\|u\|_{2}:=(u, u)^{1 / 2} .
$$

Since $\mathrm{D}_{n, p}$ is finite, these are always well-defined; we will also make occasional use of the norm

$$
\|u\|_{\infty}:=\max _{e \in \mathrm{D}_{n, p}}|u(e)| .
$$

We denote the subspace of $p$-forms vanishing on $\operatorname{Ext}\left(D_{n, p}\right)$

$$
\mathscr{W}_{0}\left(\mathrm{D}_{n, p}\right):=\left\{u \in \mathscr{W}\left(\mathrm{D}_{n, p}\right) \mid u=0 \text { on } \operatorname{Ext}\left(\mathrm{D}_{n, p}\right)\right\},
$$

which is clearly a vector space, and the bilinear form

$$
((u, v)):=\int_{\mathrm{D}_{n, 1}} \mathbf{d} u \mathbf{d} v
$$

is a well-defined inner product on $\mathscr{W}_{0}\left(\mathrm{D}_{n, 0}\right)$. $\mathscr{W}_{0}\left(\mathrm{D}_{n, 0}\right)$ is thus a Hilbert space with the corresponding norm, denoted $\|u\|_{1,2}:=((u, u))^{1 / 2}$. We now demonstrate positive-definiteness of the inner product, since we will use the resulting version of Poincaré inequality below.

Since $\mathrm{D}_{n}$ is path-connected, for any $e \in \operatorname{Int}\left(\mathrm{D}_{n, 0}\right)$, there exists $\gamma \subset \mathrm{D}_{n, 1}$ such that $\partial \gamma=e \cup-e^{\prime}$, with $e^{\prime} \in \operatorname{Ext}\left(\mathrm{D}_{n, 0}\right)$ and $\# \gamma \leqq C_{0}^{\mathrm{L}} n \operatorname{diam}(\mathrm{D})$. For any $u \in \mathscr{W}_{0}\left(\mathrm{D}_{n, 0}\right)$, we then have $u(e)=\int_{\gamma} \mathbf{d} u$, so applying the Cauchy-Schwarz inequality, we have

$$
|u(e)|^{2}=\left|\int_{\gamma} \mathbf{d} u\right|^{2} \leqq \# \gamma \int_{\gamma}|\mathbf{d} u|^{2} \leqq \# \gamma \int_{\mathrm{D}_{n, 1}}|\mathbf{d} u|^{2} .
$$


Integrating over $\mathrm{D}_{n, 0}$, and noting that there exists a constant $C_{1}^{\mathrm{L}}>0$ which depends only on the underlying lattice $\mathrm{L}$ such that $\# \mathrm{D}_{n, 0} \leqq C_{1}^{\mathrm{L}} n^{2} \operatorname{diam}(\mathrm{D})^{2}$, we have

$$
\int_{\mathrm{D}_{n, 0}}|u|^{2} \leqq C_{2}^{\mathrm{L}} n^{3} \operatorname{diam}(\mathrm{D})^{3} \int_{\mathrm{D}_{n, 1}}|\mathbf{d} u|^{2},
$$

where $C_{2}^{\mathrm{L}}=C_{0}^{\mathrm{L}} C_{1}^{\mathrm{L}}$. We note that the same inequality also holds for $u \in \mathscr{W}_{0}\left(\mathrm{D}_{n, 0}^{*}\right)$ by a similar argument.

2.4.5. Duality for $p$-Forms on Lattice Subcomplexes We define the duality mapping $*: \mathscr{W}\left(n \mathrm{D}_{n, p}\right) \rightarrow \mathscr{W}_{0}\left(\mathrm{D}_{n, 2-p}^{*}\right)$ as follows:

$$
u^{*}(a)= \begin{cases}u(e) & a=e^{*} \in \operatorname{Int}\left(\mathrm{D}_{n, 2-p}^{*}\right) \\ 0 & a \in \operatorname{Ext}\left(\mathrm{D}_{n, 2-p}^{*}\right)\end{cases}
$$

We note that this mapping is well-defined since as noted in Section 2.4.1, $a \in$ $\operatorname{Ext}\left(\mathrm{D}_{n, 2-p}^{*}\right)$ if and only if there exists no $e \in \mathrm{D}_{p}$ with $a=e^{*}$. This duality mapping defines an isomorphism from $\mathscr{W}\left(D_{n, p}\right)$ to $\mathscr{W}_{0}\left(\mathrm{D}_{n-p}^{*}\right)$ as vector spaces; as, in addition

$$
(u, u)=\int_{n \mathrm{D}_{n, p}}|u|^{2}=\int_{\operatorname{Int}\left(n \mathrm{D}_{n, p}^{*}\right)}\left|u^{*}\right|^{2}=\int_{n \mathrm{D}_{n, p}^{*}}\left|u^{*}\right|^{2}=\left(u^{*}, u^{*}\right),
$$

it follows that $*$ defines an isometry of the spaces $\mathscr{L}^{2}\left(n \mathrm{D}_{n, p}\right)$ to $\mathscr{L}^{2}\left(\mathrm{D}_{n, 2-p}^{*}\right)$. Moreover, for any $e \in n \mathrm{D}_{n, p}$, we verify that

$$
\begin{aligned}
& \mathbf{d} u(e)=\int_{\partial e} u=\int_{(\partial e)^{*}} u^{*}=\int_{\delta^{*} e^{*}} u^{*}=\delta^{*} u^{*}\left(e^{*}\right), \quad \text { and } \\
& \delta u(e)=\int_{\delta e} u=\int_{(\delta e)^{*}} u^{*}=\int_{\partial^{*} e^{*}} u^{*}=\mathbf{d}^{*} u^{*}\left(e^{*}\right) .
\end{aligned}
$$

\subsection{Dislocation Configurations}

We now recall some definitions from [31] which will permit us to give a kinematic description of screw dislocations in the setting of our model. Given $u \in \mathscr{W}\left(\mathrm{D}_{n, 0}\right)$, we define the associated set of bond-length 1-forms

$$
[\mathbf{d} u]:=\left\{\alpha \in \mathscr{W}\left(\mathrm{D}_{n, 1}\right) \mid\|\alpha\|_{\infty} \leqq \frac{1}{2}, \alpha-\mathbf{d} u \in \mathbb{Z}\right\} .
$$

A dislocation core is any positively-oriented 2-cell $e \in \mathrm{D}_{2}$ such that

$$
\mathbf{d} \alpha(e)=\int_{\partial e} \alpha \neq 0 .
$$

Let $\mu \in \mathscr{W}\left(\mathrm{D}_{2}\right)$, with $\mu: \mathrm{D}_{2} \rightarrow\{-1,0,+1\}$. We will say that $u$ is a deformation containing the dislocation configuration $\mu$ if

$$
\exists \alpha \in[\mathbf{d} u] \text { such that } \mathbf{d} \alpha=\mu .
$$


The 2-form $\mu$ represents the Burgers vectors of the dislocations in the configuration, which are the topological 'charge' of dislocations; see [30,33] for general discussion of the notion of the Burgers vector and its importance in the study of dislocations, and $[4,31]$ for further discussion of the physical interpretation of this specific definition.

For the purposes of our analysis, we define sets of admissible dislocation configurations. For $\varepsilon>0, n \in \mathbb{N}$, and $b_{i} \in\{ \pm 1\}$ for $i=1, \ldots, m$, we define the set $\mathscr{M}_{n}^{\varepsilon}\left(b_{1}, \ldots, b_{m}\right)$ of 2 -forms

$$
\begin{gathered}
\mathscr{M}_{n}^{\varepsilon}\left(b_{1}, \ldots, b_{m}\right):=\left\{\mu=\sum_{i=1}^{m} b_{i} \mathbb{1}_{e_{i}} \mid e_{i} \in \mathrm{D}_{2} \text { positively oriented, } \operatorname{dist}\left(e_{i}, \operatorname{Ext}\left(\mathrm{D}_{n, 0}\right)\right) \geqq n \varepsilon,\right. \\
\left.\operatorname{dist}\left(e_{i}, e_{j}\right) \geqq \epsilon n, \text { for all } i, j \in\{1, \ldots, m\}, i \neq j\right\} .
\end{gathered}
$$

Each 2-form in this set represents a collection of $m$ dislocations with respective Burgers vectors $b_{1}, \ldots, b_{m}$ and cores $e_{1}, \ldots, e_{m}$ : these dislocations are separated from each other and from the boundary by a distance of at least $\varepsilon n$. Since we will assume that the number of dislocations $m$, and the Burgers vectors $b_{1}, \ldots, b_{m}$ are fixed throughout, we will suppress the dependence on $\left(b_{1}, \ldots, b_{m}\right)$ from now on.

\section{Main Results}

\subsection{Energy and Equilibria}

As stated in the introduction, we follow $[1,2,4,31,32,41]$ and consider a nearestneighbour anti-plane lattice model for the cylinder of crystal. Let $\psi: \mathbb{R} \rightarrow \mathbb{R}$ be given by $\psi(x):=\frac{1}{2} \lambda \operatorname{dist}(x, \mathbb{Z})^{2}$; we consider the energy difference functional

$$
E_{n}(y ; \tilde{y}):=\int_{\mathrm{D}_{n, 1}}[\psi(\mathbf{d} y)-\psi(\mathbf{d} \tilde{y})] .
$$

This functional is a model for potential energy per unit length of a long cylindrical crystal, and points $\mathrm{D}_{n, 0}$ correspond to columns of atoms which are assumed to be periodic in the direction perpendicular to the plane considered. For further motivation of this model, we refer the reader to Section 1 of [1].

Following Definition 1 of [31], we will say that $y \in \mathscr{W}\left(\mathrm{D}_{n, 0}\right)$ is a locally stable equilibrium if there exists $\epsilon>0$ such that

$$
E_{n}(y+u ; y) \geqq 0 \quad \text { whenever }\|u\|_{1,2} \leqq \varepsilon .
$$

Due to the periodicity of $\psi$, we note that any locally stable equilibrium generates an entire family of equilibria: letting $z \in \mathscr{W}\left(\mathrm{D}_{n, 0}\right)$ taking values in $H+\mathbb{Z}$ for some $H \in \mathbb{R}$, if $y$ is a locally stable equilibrium, then so is $y+z$. These equilibria are physically indistinguishable, since they correspond to a vertical 'shifts' of columns by an integer number of lattice spacings, and a rigid vertical translation of the entire crystal by $H$. We therefore define the equivalence relation $u \sim v$ if and only if $u=v+z, \quad$ where $z: \mathrm{D}_{n, 0} \rightarrow \mathbb{Z}+H$ for some $H \in \mathbb{R}$, 
and denote the equivalence classes of this relation as $\llbracket y \rrbracket$.

We recall that Theorem 3.3 in [31] gives sufficient conditions such that locally stable equilibra containing dislocations exist in the case of a more general choice of $\psi$ than that chosen here. Our first main result is similar, but in addition provides a very precise representation of the corresponding bond-length 1 -form in the case considered here, and asserts the uniqueness (up to lattice symmetries) of local equilibria containing a given dislocation configuration.

Theorem 3.1. Fix $\varepsilon>0$ and $\mathrm{D}$ a convex lattice polygon; then for all $n$ sufficiently large, the following statements hold:

(1) For every 2-form $\mu \in \mathscr{M}_{n}^{\varepsilon}$, there exists a corresponding locally stable equilibrium $u_{\mu}$ which contains the dislocation configuration $\mu$;

(2) Each such equilibrium $u_{\mu}$ is unique up to the equivalence relation defined in (3.1); and

(3) For any $u \in \llbracket u_{\mu} \rrbracket$, there is a unique bond-length 1-form $\alpha \in[\mathbf{d} u]$ satisfying $\alpha^{*}=\mathbf{d}^{*} G_{\mu^{*}}$, where $\mu^{*}$ is the 0 -form dual to $\mu$, and $G_{\mu^{*}} \in \mathscr{W}_{0}\left(\mathrm{D}_{n, 0}\right)$ is the solution to

$$
\boldsymbol{\Delta}^{*} G_{\mu^{*}}=\mu^{*} \text { in } \operatorname{Int}\left(\mathrm{D}_{n, 0}^{*}\right), \text { with } G_{\mu^{*}}=0 \text { on } \operatorname{Ext}\left(\mathrm{D}_{n, 0}^{*}\right)
$$

Strategy of Proof The proof of this theorem is the main focus of Section 4. We begin by showing that if $u$ is a locally stable equilibrium containing dislocations $\mu$, then $\alpha \in[\mathbf{d} u]$ must necessarily satisfy

$$
\|\alpha\|_{\infty}<\frac{1}{2}, \quad \mathbf{d} \alpha=\mu \text { on } \mathrm{D}_{2}, \quad \text { and } \quad \delta \alpha=0 \text { on } \mathrm{D}_{n, 0} .
$$

We show that these conditions are satisfied by at most one $\alpha \in \mathscr{W}\left(\mathrm{D}_{n, 1}\right)$, and using the duality transformation described in Section 2.2, we verify that $\alpha \in \mathscr{W}\left(\mathrm{D}_{n, 1}\right)$ satisfying $\alpha^{*}=\mathbf{d}^{*} G_{\mu^{*}}$ verifies the latter two conditions. Showing that $\|\alpha\|_{\infty}=$ $\left\|\mathbf{d}^{*} G_{\mu^{*}}\right\|_{\infty}<\frac{1}{2}$ is the most technical aspect of the proof, and requires us to develop a theory which is analogous to obtaining interior estimates for solutions of a boundary value problem for Poisson's equation in the continuum setting. To conclude, we obtain the class $\llbracket u_{\mu} \rrbracket$ by 'integrating' $\alpha$.

\subsection{Energy Barriers}

Let $\mathrm{C}\left([0,1] ; \mathscr{W}\left(\mathrm{D}_{n, 0}\right)\right)$ denote the space of continuous paths from $[0,1]$ to $\mathscr{W}\left(\mathrm{D}_{n, 0}\right)$. For $\mu$ and $v \in \mathscr{M}_{n}^{\varepsilon}$, we define the set of continuous paths which move any local equilibrium in $\llbracket u_{\mu} \rrbracket$ to any other local equilibrium in $\llbracket u_{v} \rrbracket$ to be

$$
\begin{aligned}
& \Gamma_{n}(\mu \rightarrow \nu):=\left\{\gamma \in \mathrm{C}\left([0,1] ; \mathscr{W}\left(\mathrm{D}_{n, 0}\right)\right) \mid \gamma(0) \in \llbracket u_{\mu} \rrbracket, \gamma(1) \in \llbracket u_{\nu} \rrbracket,\right. \\
& \forall t \in[0,1], \alpha \in[\mathbf{d} \gamma(t)] \text { implies } \mathbf{d} \alpha=\mu \text { or } \mathbf{d} \alpha=v\} .
\end{aligned}
$$

In the case where we will apply this definition, i.e. where $v-\mu=b_{i}\left[\mathbb{1}_{q}-\mathbb{1}_{p}\right]$ with $q^{*}=p^{*}+\mathrm{a}^{*}$ for some nearest-neighbour direction $\mathrm{a}^{*}$ in the dual lattice, corresponding to a single dislocation 'hopping' to an adjacent site, the final condition on the paths in the above definition ensures that the Burgers vectors of the 
configurations along the path vary only on the 2-cells $p$ and $q$. In other words, we make the modelling assumption that dislocations move strictly from one site to an adjacent site, and not via a more complicated route. be

We define the energy barrier for the transition from $\mu$ to $v$ for $\mu, v \in \mathscr{M}_{n}^{\varepsilon}$ to

$$
\mathcal{B}_{n}(\mu \rightarrow v):=\min _{\gamma \in \Gamma_{n}(\mu \rightarrow v)} \max _{t \in[0,1]} E_{n}\left(\gamma(t) ; u_{\mu}\right) .
$$

Our second main result concerns an asymptotic representation of this quantity.

Theorem 3.2. Suppose that $\mu, v \in \mathscr{M}_{n}^{\varepsilon}$ are 2-forms such that $v-\mu=b_{i}\left[\mathbb{1}_{q}-\mathbb{1}_{p}\right]$ for some $i$, where $q^{*}=p^{*}+\mathrm{a}^{*}$ for some nearest neighbour direction $\mathrm{a}^{*}$ in $\mathrm{L}^{*}$. For $i=1, \ldots, m$, let $x_{i} \in \mathrm{D}$ be such that $\operatorname{dist}\left(x_{i}, \frac{1}{n} e_{i}^{*}\right) \leqq \frac{1}{n}$. Then there exist a constant $c_{0}$ which depends only on the underlying lattice complex $\mathrm{L}$ such that

$\mathcal{B}_{n}(\mu \rightarrow v)=\lambda c_{0}+\frac{1}{2} \lambda n^{-1}\left[b_{i}^{2} \nabla \bar{y}_{j}\left(x_{j}\right) \cdot \mathrm{a}^{*}+\sum_{i \mid i \neq j} b_{j} b_{i} \nabla \mathcal{G}_{x_{i}}\left(x_{j}\right) \cdot \mathrm{a}^{*}\right]+o\left(n^{-1}\right)$, where

(1) $\lambda$ is given in the definition of $\psi$,

(2) $\bar{y}_{j}$ solves the boundary value problem

$$
\Delta \bar{y}_{j}=0 \text { in } \mathrm{D}, \quad \bar{y}_{j}(\cdot)=\frac{1}{\mathcal{V}_{\pi}} \log \left(\left|\cdot-x_{j}\right|\right) \text { on } \partial \mathrm{D},
$$

(3) $\mathcal{G}_{y}$ is the solution to

$$
\Delta \mathcal{G}_{y}=\frac{\mathcal{V}}{2} \delta_{y} \text { in } \mathrm{D}, \text { with } \mathcal{G}_{y}=0 \text { on } \partial \mathrm{D},
$$

where we recall the definition of $\mathcal{V}$ from (2.5), and

(4) $o\left(n^{-1}\right)$ satisfies $n o\left(n^{-1}\right) \rightarrow 0$ as $n \rightarrow \infty$, uniformly for all $\mu \in \mathscr{M}_{n}^{\varepsilon}$.

Strategy of Proof The proof of this result is the main focus of Section 5. Our main task is the explicit construction of a transition state, i.e. $u_{\downarrow} \in \mathscr{W}\left(\mathrm{D}_{n, 0}\right)$ such that

$$
E_{n}\left(u_{\downarrow} ; u_{\mu}\right)=\min _{\gamma \in \Gamma_{n}(\mu \rightarrow \nu)} \max _{t \in[0,1]} E_{n}\left(\gamma(t) ; u_{\mu}\right) .
$$

This may be seen as a generalisation of the notion of a critical point, but is not a true critical point, since $E_{n}$ is not differentiable at $u_{\downarrow}$. Nevertheless, we show that $\alpha \in\left[\mathbf{d} u_{\downarrow}\right]$ has a dual which is closely related to the interpolation of $\mathbf{d}^{*} G_{\mu^{*}}$ and $\mathbf{d}^{*} G_{v^{*}}$ which are solutions of (3.2). This dual representation, combined with the precise asymptotics obtained for $\mathbf{d}^{*} G_{\mu^{*}}$ in order to prove Theorem 3.1, allow us to derive the expression of $\mathcal{B}_{n}(\mu \rightarrow \nu)$.

\subsection{Remarks on the Model}

Here, we collect a few remarks concerning the choice of model, the notion of duality we use, and some further links between the results above and the way in which dislocations are modelled in continuum elastoplasticity. 
More General Potentials The derivation of the energy we consider as given in Section 2.2 of [2] suggests that potential $\psi$ should be chosen to be smooth, in keeping with the usual assumptions on interatomic potentials. On the other hand, our results rely heavily on the definition of $\psi$, since the structure of the potential chosen permits us both to prove the characterisation and uniqueness of $\alpha$ given in Theorem 3.1, and to be precise about the set on which $\mathcal{B}(\mu \rightarrow v)$ is attained. This ultimately provides us with a means by which to prove Theorem 3.2.

In spite of this, a result similar to Theorem 3.2 may hold in cases where $\psi$ is more general, but is sufficiently 'close' to the choice made here (see for example the structural assumptions made in Section 5 of [2]). Since the interatomic distances rapidly approach those predicted by linear elasticity as one moves away from a dislocation core (see Theorem 3.5 in [17]), and much of the potential energy is carried by the elastic field at significant distances from the dislocation core where a harmonic approximation of the energy is valid, heuristically one might expect that the energy barrier should be similar to that given in Theorem 3.2. However, due to the complexity of possible transitions in a more general case, such a result does not seem tractable without very strong assumptions on the potential, and significant additional technicalities: we therefore do not pursue such results here.

Dynamics in the Infinite Lattice We remark that a significant amount of our analysis is devoted to verifying the first condition in (3.3) holds. This aspect of the proof of Theorem 3.1 would be significantly simplified if we were to consider the problem in an infinite domain, since in this case integral representations of the lattice Green's function are available via Fourier-analysis. Nevertheless, we pursue the evolution on a finite domain here, both because this is a case of physical relevance, and because we are able to demonstrate that the boundary affects the evolution of the configuration in exactly the manner described in Section 2.1 of [46].

Equilibrium Conditions and Geometry Finally, we remark that the two latter conditions in (3.3) are analogous to the requirement that a continuum strain field $\varepsilon$ satisfies

$$
\operatorname{curl}(\varepsilon)=\mu \text { and } \operatorname{div}(\mathbb{C}: \varepsilon)=0 .
$$

These are the conditions usually prescribed on a strain field $\varepsilon$ which contain dislocations described by a measure $\mu$ in a linear elastic setting (see for example (1.1) in [14]).

We also note that the precise notion of duality which we use is specific to twodimensional modelling of dislocations, as it is only in this case that $L_{1}$ and $L_{1}^{*}$ are related by duality. The fact that dual 1-cells are orthogonal segments suggest that one should view the construction of $\alpha$ by duality as a version of the Cauchy-Riemann equations for harmonic conjugate functions.

\subsection{KMC Model for Dislocation Motion}

With the asymptotic expression for $\mathcal{B}_{n}(\mu \rightarrow \nu)$ given by Theorem 3.2, we are now in a position to apply (1.1) and formulate the KMC model for dislocation 
motion we wish to study. In doing so, we make several modeling assumptions, which we now discuss in detail.

Our first assumption is that the only possible transitions are from $\mu \in \mathscr{M}_{n}^{\varepsilon}$ to $v \in \mathscr{M}_{n}^{\varepsilon}$ satisfying

$$
\begin{aligned}
& v-\mu=b_{i}\left[\mathbb{1}_{q}-\mathbb{1}_{p}\right] \text { for some } i \in\{1, \ldots, m\}, \\
& \text { with } p^{*}=q^{*}+\mathrm{a}^{*} \text { for some dual lattice nearest-neighbour direction } \mathrm{a}^{*} .
\end{aligned}
$$

This requirement prevents the following possible situations from arising:

(1) Multiple dislocations cannot move together in a coherent way: it seems reasonable to dismiss this possibility since we consider a regime where dislocations are far apart.

(2) Single dislocations cannot make successive correlated jumps over several lattice sites. Since we consider a low temperature regime, we expect the probability of multiple correlated jumps to be negligible.

(3) Dislocations cannot be spontaneously generated in the material during the course of the evolution. In this case, we expect the energy barrier for dipole creation to be higher than that for the motion of single dislocations, so once again, we expect such events to be of very small probability and we therefore neglect them.

We therefore assume that the transition time for a dislocation $\mu$ to $v$ is exponentially distributed with rate

$$
\mathcal{R}_{n}(\mu \rightarrow \nu):=\mathcal{A}_{n}(\mu \rightarrow v) \exp \left(-\beta \mathcal{B}_{n}(\mu \rightarrow \nu)\right)
$$

where:

(1) $\mathcal{B}_{n}(\mu \rightarrow v)$ is the energy barrier for the transition from $\mu$ to $v$ defined by (3.4),

(2) $\beta=\left(k_{B} T\right)^{-1}$ is the inverse thermodynamic temperature, and

(3) $\mathcal{A}_{n}(\mu \rightarrow v)$ is the pre-exponential rate factor which is related to the entropic 'width' of the pathways connecting $\mu$ and $\nu$, and hence also depends on the inverse temperature $\beta$.

Formula (1.1) may be interpreted as follows: the exponential factor encodes the probability that thermal fluctuations will result in the system achieving the potential energy necessary for a transition to happen. The prefactor then determines how often such energy levels will lead to a transition: if the passage between states in the energy landscape is very 'narrow', then even if the system achieves sufficient energy to exit, it may only rarely find the the pathway to achieve such a transition.

Our second main assumption will be that $\mathcal{A}_{n}(\mu \rightarrow v)=\mathcal{A}_{0}+o(1)$, as $\beta \rightarrow \infty$ and $n \rightarrow \infty$, where $\mathcal{A}_{0}$ is independent of $\mu$ and $\nu$. In the case of a finite-dimensional system with a smooth potential energy $V$, having local minima at $x$ and $y$, and a saddle point at $z$ with a single unstable direction where the minimal energy barrier between $x$ and $y$ is achieved, the form of the prefactor is [see formula (25) in [34] 
for the original one-dimensional derivation, or [27] for an overview of variants derived in a variety of situations]

$$
\mathcal{A}(\mu \rightarrow \nu)=\frac{\sqrt{\gamma^{2}+4\left|\lambda_{1}(z)\right|}-\gamma}{2 \pi} \sqrt{\frac{\operatorname{det} \nabla^{2} V(x)}{\left|\operatorname{det} \nabla^{2} V(z)\right|}}+o(1) .
$$

Here $\gamma$ is a friction coefficient, with units of time ${ }^{-1}$, and $\lambda_{1}(z)$ is the eigenvalue of the Hessian at $z$ which corresponds to the unstable direction. The rate can be reduced if either the eigenvalues of $\nabla^{2} V(x)$ are made smaller, reducing its determinant, or if the positive eigenvalues of $\nabla^{2} V(z)$ are increased. The former means the potential energy 'basin' around $x$ is wider, and the latter means that the 'mountain pass' in the energy landscape through which the system can travel most easily to arrive at state $v$ is narrower. This coefficient therefore encodes entropic effects related to the shape of the energy landscape.

In our model, we have shown that there is a discontinuity in the first derivative at the energy barrier between states, so the exact expression (3.5) cannot be valid; however, in directions for which second derivatives exist, the Hessian of the energy at the transition state and at equilibria are identical, motivating the assumption that $\mathcal{A}_{n}$ is constant as $n \rightarrow \infty$ and $\beta \rightarrow \infty$. We remark that it is usual in practice (except in symmetric situations where multiple transition pathways with the same energy barrier exist) to choose a constant prefactor in KMC simulations, since eigenvalue decompositions of the Hessian of the energy are often unavailable, and transition events may be too rare to obtain a sufficiently accurate numerical estimate of the rate. In order to describe the limit, we define the set of admissible (macroscale) dislocation positions to be

$$
\mathscr{M}_{\infty}^{\varepsilon}:=\left\{\left(x_{1}, \ldots, x_{m}\right) \in \mathrm{D}^{m}\left|x_{i} \in \mathrm{D},\right| x_{i}-x_{j} \mid \geqq \varepsilon, \operatorname{dist}\left(x_{i}, \partial \mathrm{D}\right) \geqq \varepsilon, \forall i, j \text { with } i \neq j\right\},
$$

and identify $\mathscr{M}_{n}^{\varepsilon}$ with a subset of this space by the embedding

$$
\iota_{n}: \mathscr{M}_{n}^{\varepsilon} \rightarrow \mathscr{M}_{\infty}^{\varepsilon}, \quad \text { where } \iota_{n}\left(\sum_{i=1}^{m} b_{i} \mathbb{1}_{e_{i}}\right)=\left(\frac{1}{n} e_{1}^{*}, \ldots, \frac{1}{n} e_{m}^{*}\right)
$$

It is clear that this map is well-defined, and by endowing $\mathscr{M}_{n}^{\varepsilon}$ with the metric

$$
r_{n}(\mu, v)=\sum_{i=1}^{m} \frac{1}{n} \operatorname{dist}\left(e_{i}^{*},\left(e_{i}^{\prime}\right)^{*}\right) \quad \text { where } \quad \mu=\sum_{i=1}^{m} b_{i} \mathbb{1}_{e_{i}} \text { and } v=\sum_{i=1}^{m} b_{i} \mathbb{1}_{e_{i}^{\prime}},
$$

and $\mathscr{M}_{\infty}^{\varepsilon}$ with the metric

$$
r_{\infty}(\mu, v)=\sum_{i=1}^{m} \operatorname{dist}\left(x_{i}, x_{i}^{\prime}\right) \text { where } \mu=\left(x_{1}, \ldots, x_{m}\right) \text { and } v=\left(x_{1}^{\prime}, \ldots, x_{m}^{\prime}\right)
$$

$\iota_{n}$ is an isometric embedding. It is straightforward to see that each of these spaces is compact. 
Given a differentiable function $f: \mathscr{M}_{\infty}^{\varepsilon} \rightarrow \mathbb{R}$, we will write $\partial_{i} f(x)$ to mean the $\mathbb{R}^{2}$-valued function such that

$\partial_{i} f(x) \cdot \mathbf{a}=f\left(x_{1}, \ldots, x_{i}+\mathrm{a}, \ldots, x_{m}\right)-f\left(x_{1}, \ldots, x_{m}\right)+o(|\mathrm{a}|)$ for all $\mathrm{a} \in \mathbb{R}^{2}$.

Let $\mathrm{D}\left([0, T] ; \mathscr{M}_{n}^{\varepsilon}\right)$ denote the Skorokhod space of càdlàg maps from $[0, T] \subset$ $\mathbb{R}$ with values in $\mathscr{M}_{n}^{\varepsilon}$, and denote the space of continuous real-valued functions defined on $\mathscr{M}_{n}^{\varepsilon}$ to be $\mathrm{C}\left(\mathscr{M}_{n}^{\varepsilon} ; \mathbb{R}\right)$ : this is in fact the space of all real-valued functions on $\mathscr{M}_{n}^{\varepsilon}$, since the metric $r_{n}$ induces the discrete topology. Define

$$
\mathcal{N}_{\mu}:=\left\{v \in \mathscr{M}_{n}^{\varepsilon} \mid r_{n}(\mu, v)=d^{\mathrm{L}}\right\}, \quad \text { where } \quad d^{\mathrm{L}}=\left\{\begin{aligned}
\frac{\sqrt{3}}{3} \mathrm{~L} & =\mathrm{Tr} \\
1 \mathrm{~L} & =\mathrm{Sq} \\
\sqrt{3} \mathrm{~L} & =\mathrm{Hx}
\end{aligned}\right.
$$

Since we expect our modelling assumptions to break down as dislocations either approach one another or the domain boundary, we stop the evolution in such an event. We therefore denote what we term the boundary of $\mathscr{M}_{n}^{\varepsilon}$, defined to be

$$
\partial \mathscr{M}_{n}^{\varepsilon}:=\left\{\mu=\sum_{i=1}^{m} b_{i} \mathbb{1}_{e_{i}} \in \mathscr{M}_{n}^{\varepsilon} \mid \exists v \notin \mathscr{M}_{n}^{\varepsilon} \text { such that } r_{n}(\mu, v)=d^{\mathrm{L}}\right\} \text {. }
$$

We consider the sequence of Markov processes $Y^{n} \in D\left([0, T] ; \mathscr{M}_{n}^{\varepsilon}\right)$ which are killed on the boundary $\partial \mathscr{M}_{n}^{\varepsilon}$, having infinitesimal generator $\Omega_{n}: \mathrm{C}\left(\mathscr{M}_{n}^{\varepsilon} ; \mathbb{R}\right) \rightarrow$ $\mathrm{C}\left(\mathscr{M}_{n}^{\varepsilon} ; \mathbb{R}\right)$ where

$$
\left[\Omega_{n} f\right](\mu):= \begin{cases}\sum_{\nu \in \mathcal{N}_{\mu}} \mathcal{T}_{n} \mathcal{R}_{n}(\mu \rightarrow v)[f(\nu)-f(\mu)], & \mu \in \mathscr{M}_{n}^{\varepsilon} \backslash \partial \mathscr{M}_{n}^{\varepsilon}, \\ 0 & \mu \in \partial \mathscr{M}_{n}^{\varepsilon},\end{cases}
$$

and $\mathcal{R}_{n}(\mu \rightarrow \nu)$ is defined in (1.1). Since $\mathcal{R}_{n}(\mu \rightarrow \nu)$ is strictly positive and bounded for all $\mu, v \in \mathscr{M}_{n}^{\varepsilon}$ and $n \in \mathbb{N}, \Omega_{n}$ is a bounded linear operator. Defining $X_{t}^{n}:=\iota_{n}\left(Y_{t}^{n}\right)$, it follows that $X_{t}^{n}$ is a Markov process on the space $\mathscr{M}_{\infty}^{\varepsilon}$.

\subsection{The Feng-Kurtz Approach to Large Deviations Principles}

The last of our main results will be to show that in a specific asymptotic regime, the Markov processes $X^{n}$ satisfy a Large Deviations Principle. To do so, we apply the general theory developed in [21], which provides an approach to proving such results by demonstrating the convergence of a sequence of nonlinear semigroups. For convenience, we provide the following theorem as a synthesis of the results of Theorem 6.14 and Corollary 8.29 in [21], adapted to our application.

Theorem 3.3. Suppose that the following conditions hold:

(1) $M$ is a compact subset of $\mathbb{R}^{N}$, viewed a metric space with the usual metric induced by the Euclidean norm. 
(2) For all $n \in \mathbb{N},\left(M_{n}, r_{n}\right)$ is a complete separable metric space and there exists a sequence $\iota_{n}: M_{n} \rightarrow M$ of Borel measurable maps such that for any $x \in M$, there exists $z_{n} \in M_{n}$ satisfying $\iota_{n}\left(z_{n}\right) \rightarrow x$.

(3) For each $n \in \mathbb{N}, \Omega_{n}: \mathrm{C}\left(M_{n} ; \mathbb{R}\right) \rightarrow \mathrm{C}\left(M_{n} ; \mathbb{R}\right)$ is the infinitesimal generator of a Markov process on $M_{n}$. Suppose the martingale problem is well-posed, i.e. for any initial distribution $\mu_{0}$ on $M_{n}$, the distribution of the Markov process at all later times is uniquely determined, and the mapping from $y \in M_{n}$ to trajectories with initial distribution $\delta_{y}$ is Borel measurable under the weak topology on the space of probability measures defined on $\mathrm{D}\left([0,+\infty) ; M_{n}\right)$.

(4) For any $n \in \mathbb{N}$, and any $f \in \mathrm{C}\left(M_{n} ; \mathbb{R}\right)$, define the nonlinear generator

$$
H_{n} f(x):=\frac{1}{n} \mathrm{e}^{-n f(x)}\left[\Omega_{n} \mathrm{e}^{n f}\right](x) .
$$

Let $H$ be an operator mapping $\mathrm{C}^{1}(M ; \mathbb{R})$ to the space of bounded measurable functions on $M$, which is represented as

$$
H f(x)=\mathcal{H}(x, \nabla f(x)),
$$

where $\mathcal{H}: M \times \mathbb{R}^{N} \rightarrow \mathbb{R}$ satisfies the following conditions:

- $\mathcal{H}$ is uniformly continuous on the interior of $M \times B_{r}(0)$ for all $r>0$,

- $\mathcal{H}$ is differentiable in $p$ on the interior of $M \times \mathbb{R}^{N}$,

- $\mathcal{H}(x, p)=0$ for all $p \in \mathbb{R}^{N}$ when $x \in \partial M$, and

- For all $x \in M, p \mapsto \mathcal{H}(x, p)$ is a convex function.

For each pair $(f, g)$ such that $g=H f$, there exists a sequence $\left(f_{n}, g_{n}\right)$ such that $g_{n}=H_{n} f_{n}$, $\left\|f \circ \iota_{n}-f_{n}\right\| \rightarrow 0, g_{n}$ is uniformly bounded, and for any sequence $z_{n} \in M_{n}$ satisfying $\iota_{n}\left(z_{n}\right) \rightarrow x$, we have

$$
g^{l}(x) \leqq \liminf _{n \rightarrow \infty} g_{n}\left(z_{n}\right) \leqq \limsup _{n \rightarrow \infty} g_{n}\left(z_{n}\right) \leqq g^{u}(x),
$$

where $g^{l}$ and $g^{u}$ are respectively the lower and upper-semicontinuous regularizations of $g$,

$$
g^{l}(x):=\lim _{r \rightarrow 0} \inf _{y \in B_{r}(x)} g(y) \text { and } g^{u}(x):=\lim _{r \rightarrow 0} \sup _{y \in B_{r}(x)} g(y) .
$$

(5) There exists $\mathcal{L}: M \times \mathbb{R}^{N} \rightarrow[0,+\infty]$ such that

$$
\begin{gathered}
\mathcal{L}(x, \xi)=\sup _{p \in \mathbb{R}^{N}}\{\xi \cdot p-\mathcal{H}(x, p)\}, \\
\lim _{|\xi| \rightarrow \infty} \frac{\mathcal{L}(x, \xi)}{|\xi|}=+\infty \text { for all } x \in M \text { and } \xi \in \mathbb{R}^{N}
\end{gathered}
$$

and for each $x_{0} \in M$, there exists $x \in \mathrm{W}^{1,1}\left([0, T] ; \mathbb{R}^{N}\right)$ satisfying $x(0)=x_{0}$ and

$$
\int_{0}^{T} \mathcal{L}(x(t), \dot{x}(t)) \mathrm{d} t=0
$$


Then the sequence of $M$-valued processes $X_{n}:=\iota_{n}\left(Y_{n}\right)$ with $X_{n}(0)=\iota_{n}\left(y_{n}\right)$, where $y_{n} \in M_{n}$ and $\iota_{n}\left(y_{n}\right) \rightarrow x_{0}$ as $n \rightarrow \infty$, satisfy a Large Deviations Principle with rate functional

$$
\mathcal{J}(x):= \begin{cases}\int_{0}^{\infty} \mathcal{L}(x, \dot{x}) \mathrm{d} t & x \in \mathrm{W}^{1,1}\left([0,+\infty) ; \mathbb{R}^{N}\right) \text { with } x(0)=x_{0}, \\ +\infty & \text { otherwise }\end{cases}
$$

Section 6 contains the proof of this result, which amounts to checking that the assumptions above correspond to a series of conditions in [21].

\subsection{Asymptotics for the KMC Model}

An important condition of Theorem 3.3 is the verification of the convergence of the nonlinear generator, $H_{n}$. It will be this which motivates our particular choice of regime after we have non-dimensionalised the model. Since we are interested in the physically-relevant case of observing a large system over a long timescale, we let $\mathcal{T}_{n} \gg 1$ be the timescale of observation, which will be taken relative to the typical timescale on which a dislocation configuration changes. We then multiply all rates by this timescale, which we view as corresponding to observing the process over a long timescale.

Now, recalling the definition of the nonlinear generator given in (3.7), suppose that $f \in \mathrm{C}^{1}\left(\mathscr{M}_{\infty}^{\varepsilon} ; \mathbb{R}\right)$, and and let $x_{n}=\left(\frac{1}{n} e_{1}^{*}, \ldots, \frac{1}{n} e_{m}^{*}\right)$. By Taylor expanding $f$, we find that

$$
\begin{aligned}
& H_{n}\left(f \circ \iota_{n}\right)\left(x_{n}\right) \\
& \quad=\sum_{i=1}^{m} \sum_{j=1}^{\mathcal{K}^{*}} \frac{\mathcal{T}_{n} \mathcal{R}_{n}(\mu \rightarrow v)}{n}\left[\exp \left(\partial_{i} f\left(x_{n}\right) \cdot \mathrm{s}_{i, j}+o(1)\right)-1\right] \text { as } n \rightarrow \infty,
\end{aligned}
$$

where $\mathrm{s}_{i, j}$ are the nearest neighbour directions in $\mathrm{L}^{*}$ at $e_{i}^{*}$, and $\mathcal{K}^{*}$ is the number of nearest neighbours in $L^{*}$. Now, by applying Theorem 3.2 and the assumption that $\mathcal{A}_{n}(\mu \rightarrow \nu)=\mathcal{A}_{0}+o(1)$, we have that

$$
\begin{aligned}
& \frac{\mathcal{T}_{n} \mathcal{R}_{n}(\mu \rightarrow v)}{n}=\frac{\mathcal{T}_{n} \mathcal{A}_{0} \mathrm{e}^{-\beta \lambda c_{0}}}{n} \exp \left[-\frac{\beta \lambda}{2 n} \partial_{i} \mathcal{E}\left(x_{n}\right) \cdot \mathrm{s}_{i, j}\right]+o\left(\frac{\mathcal{T}_{n}}{n}\right), \\
& \text { where } \mathcal{E}(x):=\sum_{i=j}^{m} b_{j}^{2} \bar{y}_{j}\left(x_{j}\right)-\sum_{\substack{i, j=1 \\
i<j}}^{m} \frac{1}{2} b_{i} b_{j} \mathcal{G}_{x_{i}}\left(x_{j}\right) .
\end{aligned}
$$

Here, following [2] we have defined the renormalised energy, $\mathcal{E} .-\partial_{i} \mathcal{E}(x)$ is the Peach-Köhler force on the dislocation at $x_{i}$, and hence the gradient flow dynamics of $\mathcal{E}$ corresponds to Discrete Dislocation Dynamics. We identify two parameters in this expression,

$$
A:=\frac{\mathcal{T}_{n} \mathcal{A}_{0} \mathrm{e}^{-\beta \lambda c_{0}}}{n} \text { and } B:=\frac{\beta \lambda}{2 n},
$$

which are dimensionless, upon recalling that: 
(1) $\mathcal{T}_{n}$ has units of time,

(2) $n$ is the diameter of the domain relative to a fixed reference domain, and hence is dimensionless,

(3) $\beta=\left(k_{B} T\right)^{-1}$ is the inverse thermodynamic temperature of the system per particle,

(4) $\lambda$ has units of energy per particle, and

(5) $\mathcal{A}_{0}$ is the rate of successful exits from $\mu \rightarrow v$, and has units of time ${ }^{-1}$.

We may think of $\mathcal{A}_{0} \mathrm{e}^{-\beta \lambda c_{0}}$ as being the number of times a dislocation hops a single spacing in the full lattice per unit time, when subject to zero stress. Dividing by $n$ and multiplying by $\mathcal{T}_{n}$, this becomes the proportion of the domain crossed per proportion of time over which the system is observed. The product $\beta \lambda$ is the ratio between the potential energy required to allow transitions to occur relative to the available thermal energy; dividing by $n$ gives this quantity relative to the ratio between the lattice spacing and the domain diameter.

We therefore consider the asymptotic regime where $n \rightarrow \infty$ with $A$ and $B$ are held constant: assuming that $\lambda$ and $\mathcal{A}_{0}$ remain constant as $n, \beta$ and $\mathcal{T}_{n}$ vary, this entails that $\beta$ and $\mathcal{T}_{n}$ tend to infinity, and hence we consider a regime in which a large system is observed at low temperature for a long time. In this regime, we obtain the following result, which is proved in Section 6 as an application of Theorem 3.3. It corresponds to a rigorous validation of the equations of two-dimensional Discrete Dislocation Dynamics $[3,12,13]$ for screw dislocations in the given physical parameter regime.

Theorem 3.4. Suppose that $\mathrm{L}=\mathrm{Hx}, \mathrm{L}=\mathrm{Sq}$ or $\mathrm{L}=\operatorname{Tr}$ and $X_{0}^{n}=\iota_{n}\left(x^{n}\right)$ where $x^{n} \rightarrow x_{0} \in \mathscr{M}_{\infty}^{\varepsilon}$ as $n \rightarrow \infty$. Then the sequence of processes $X_{t}^{n}$ satisfies a Large Deviation Principle with a good rate function as $n \rightarrow \infty$ with $A$ and $B$ fixed.

Moreover, in each case, the rate function is minimised by the unique solution of the $O D E$

$$
\dot{x}=\mathcal{M}_{A, B}^{\mathrm{L}}[-\nabla \mathcal{E}(x)], \text { with } x(0)=x_{0},
$$

where $\mathcal{E}: \mathscr{M}_{\infty}^{\varepsilon} \rightarrow \mathbb{R}$ is the renormalised energy, and $\mathcal{M}_{A, B}^{\mathrm{L}}$ is the mobility function

$$
\mathcal{M}_{A, B}^{\mathrm{L}}[\xi]:=\left\{\begin{array}{r}
\sum_{i=1}^{m} \sum_{j=1}^{6} A \sinh \left(B \xi_{i} \cdot \mathrm{a}_{j}\right) \mathrm{a}_{j} \mathrm{~L}=\mathrm{Hx}, \\
\sum_{i=1}^{m} \sum_{j=1}^{4} A \sinh \left(B \xi_{i} \cdot \mathrm{e}_{j}\right) \mathrm{e}_{j} \mathrm{~L}=\mathrm{Sq}, \\
\sum_{i=1}^{m} \frac{\sum_{j=1}^{6} A \sinh \left(B \xi_{i} \cdot \mathrm{a}_{j}\right) \mathrm{a}_{j}}{\sum_{j=1}^{3} 2 \cosh \left(B \xi_{i} \cdot \frac{1}{3}\left[\mathrm{a}_{2 j}+\mathrm{a}_{2 j-1}\right]\right)} \mathrm{L}=\mathrm{Tr},
\end{array}\right.
$$

where $\mathrm{a}_{j}$ and $\mathrm{e}_{j}$ are as defined in Section 2.3.

\subsection{Generalised Gradient Flows and Mobility Functions}

As has been noted in [10,36], there is a close link between minimisers of Large Deviations rate functionals and gradient flows: we also observe this phenomenon 
here in the cases where $L=H x$ and $L=S q$. In those cases, it is shown in Section 6.2 that the rate functional takes the form

$$
\mathcal{J}_{A, B}^{\mathrm{L}}(x)=B \int_{0}^{T} \Phi_{A, B}^{\mathrm{L}}(\dot{x})+\Psi_{A, B}^{\mathrm{L}}(-\nabla \mathcal{E}(x))+\langle\nabla \mathcal{E}(x), \dot{x}\rangle \mathrm{d} t
$$

where $\mathcal{M}_{A, B}^{\mathrm{L}}=\nabla \Psi_{A, B}^{\mathrm{L}}$, and $\Phi_{A, B}^{\mathrm{L}}$ is the Legendre-Fenchel transform of $\Psi_{A, B}^{\mathrm{L}}$. This entails that the minimiser of the rate functional is a solution of a generalised gradient flow in the sense described in [37]. Furthermore, as in Theorem 3.1 of [10], we may recover a quadratic dissipation in the limit where $A \rightarrow \infty$ and $B \rightarrow 0$.

Proposition 3.5. Suppose that $A \rightarrow \infty$ and $B \rightarrow 0$ with $A B \rightarrow \omega$. Then

$$
\mathcal{M}_{A, B}^{\mathrm{L}}[\xi] \rightarrow \frac{1}{2} \omega \mathcal{V}^{*} \xi
$$

uniformly on compact subsets of $\mathbb{R}^{2 m}$, where $\mathcal{V}^{*}$ is the constant $\mathcal{V}$ for $L^{*}$. Consequently, for sufficently small $T$, solutions $x:[0, T] \rightarrow \mathrm{D}^{m}$ of (3.12) converge uniformly converge to the solution of

$$
\dot{x}=-\frac{1}{2} \omega \mathcal{V}^{*} \nabla \mathcal{E}(x), \text { with } \quad x(0)=x_{0}
$$

as $A \rightarrow+\infty$ and $B \rightarrow 0$ with $A B \rightarrow \omega$.

The proof of this result follows directly from representing $\mathcal{M}_{A, B}^{\mathrm{L}}$ via series expansion, and we omit it. Recalling the interpretation of $A$ and $B$ given in Section 3.6, this could be viewed as suggesting a Large Deviations Principle in the regime where the thermal energy is much larger than the energy barrier to dislocation motion, but where the proportion of the cylinder crossed by a dislocation during the observed time is small. However, recalling the definition of $A$ and $B$ from Section 3.4, we note that

$$
A B=\frac{\mathcal{T}_{n}}{n^{2}} \mathcal{A}_{0} \beta \lambda \exp \left(c_{0} \beta \lambda\right) .
$$

If $\beta, \lambda$ and $\mathcal{A}_{0}$ are fixed as $n \rightarrow \infty$, choosing $A B \rightarrow \omega$ corresponds to a diffusive scaling of the Markov process. We would therefore expect that randomness would persists on a macroscopic scale in such an asymptotic regime, a connection which should be explored in future work.

Finally, we remark that is also possible to consider the other scaling regime analysed in [10], in which $B \rightarrow \infty$ with $\log (A)=-c_{1} B$ for some $c_{1}$. In terms of the parameters described in Section 3.4, this entails that

$$
\log \frac{\mathcal{T}_{n} \mathcal{A}_{0}}{n}=\beta \lambda\left(c_{0}-\frac{c_{1}}{2 n}\right) .
$$

Assuming that $\lambda$ and $\mathcal{A}_{0}$ remain fixed, the only way in which this scaling regime can be attained is if $n$ remains small and fixed, with $\beta \rightarrow \infty$ and $\mathcal{T}_{n} \rightarrow \infty$. Since our analysis relies upon the fact that $n \rightarrow \infty$ to ensure that lower-order terms vanish in $\mathcal{R}_{n}(\mu \rightarrow v)$, we cannot be certain that this limit corresponds to a physically-relevant limit, and thus we do not study it here. 


\section{Proof of Theorem 3.1}

In this section, we develop discrete elliptic estimates which will allow us to prove Theorem 3.1; many of the tools used are analogous to those used in the regularity theory of scalar elliptic partial differential equations. To motivate our approach, and to provide the reader with some intuition, we recall the following result, proved in Section 3.4 of [24]: given $Q=\left\{x \in \mathbb{R}^{2}|| x \cdot \mathrm{e}_{1}||, x \cdot \mathrm{e}_{2} \mid \leqq d\right\}$, $f \in \mathrm{C}(\bar{Q})$ and $u \in \mathrm{C}^{2}(Q) \cap \mathrm{C}(\bar{Q})$ satisfying $\Delta u=f$, then

$$
\left|\nabla u(0) \cdot \mathrm{e}_{i}\right| \leqq \frac{2}{d} \sup _{\partial Q}|u|+\frac{d}{2} \sup _{Q}|f| .
$$

Our approach will be to apply the discrete analogue of the techniques used to prove this bound, i.e. the maximum principle and elementary potential theory. The application of these techniques in combination with fine residual estimates, will then allow us to conclude the proof.

\subsection{The Discrete Poisson Boundary Value Problem}

We begin by proving existence of solutions to the Poisson boundary value problem in a general path-connected subcomplex $\mathrm{D}_{n, 0}$.

Lemma 4.1. Suppose $\mathrm{D}_{n, 0}$ is a path-connected lattice subcomplex; let $g$ : $\operatorname{Ext}\left(\mathrm{D}_{n, 0}\right) \rightarrow \mathbb{R}$, and $f: \operatorname{Int}\left(\mathrm{D}_{n, 0}\right) \rightarrow \mathbb{R}$ then there exists a unique solution $u \in \mathscr{W}\left(\mathrm{D}_{n, 0}\right)$ to the problem

$$
\Delta u=f \text { in } \operatorname{Int}\left(\mathrm{D}_{n, 0}\right) \text { with } u=g \text { on } \operatorname{Ext}\left(\mathrm{D}_{n, 0}\right) .
$$

Proof. We employ a discrete version of the Dirichlet principle: extend $g$ to a 0 form by defining $g(e):=0$ for all $e \in \operatorname{Int}\left(\mathrm{D}_{n, 0}\right)$, and let $I: \mathscr{W}_{0}\left(\mathrm{D}_{n, 0}\right) \rightarrow \mathbb{R}$ be given by

$$
I(v):=\frac{1}{2}(\mathbf{d}(v+g), \mathbf{d}(v+g))-\int_{\operatorname{Int}\left(\mathrm{D}_{n, 0}\right)} f v .
$$

It is straightforward to verify that this functional is twice Gateau-differentiable, with

$$
\langle D I(v), u\rangle=(\mathbf{d}(v+g), \mathbf{d} u)-\int_{\operatorname{Int}\left(\mathrm{D}_{n, 0}\right)} f u, \quad \text { and }\left\langle D^{2} I(v) u, u\right\rangle=((u, u)) .
$$

It follows that $I$ is strictly convex, so has a unique minimiser. By setting $u=\mathbb{1}_{e}$ for any $e \in \operatorname{Int}\left(\mathrm{D}_{n, 0}\right)$, this minimiser $v$ satisfies

$$
\Delta(v+g)=f \text { in } \operatorname{Int}\left(\mathrm{D}_{n, 0}\right),
$$

and $v+g=g$ on $\operatorname{Ext}\left(\mathrm{D}_{n, 0}\right)$ by definition.

Our next auxiliary result is to prove the following discrete maximum principle. 
Lemma 4.2. Suppose that $u \in \mathscr{W}\left(\mathrm{D}_{n, 0}\right)$. Then

$$
\begin{aligned}
& \Delta u \geqq 0 \text { on } \operatorname{Int}\left(\mathrm{D}_{n, 0}\right) \quad \text { implies } \min _{e \in \mathrm{D}_{n, 0}} u(e)=\min _{e \in \operatorname{Ext}\left(\mathrm{D}_{n, 0}\right)} u(e), \quad \text { and } \\
& \Delta u \leqq 0 \text { on } \operatorname{Int}\left(\mathrm{D}_{n, 0}\right) \text { implies } \max _{e \in \mathrm{D}_{n, 0}} u(e)=\max _{e \in \operatorname{Ext}\left(\mathrm{D}_{n, 0}\right)} u(e) .
\end{aligned}
$$

Proof. We prove only the former statement, the proof of the latter being almost identical. Suppose that $u$ satisfies $\Delta u \geqq 0$ on $\operatorname{Int}\left(\mathrm{D}_{n, 0}\right)$, and that there exists $e \in \operatorname{Int}\left(\mathrm{D}_{n, 0}\right)$ such that

$$
u(e)=\min _{e^{\prime} \in \mathrm{D}_{n, 0}} u\left(e^{\prime}\right) .
$$

Either $e \in \operatorname{Ext}\left(\mathrm{D}_{n, 0}\right)$, so there is nothing to prove, or else $e \in \operatorname{Int}\left(\mathrm{D}_{n, 0}\right)$. Since

$$
\Delta u(e)=\sum_{\substack{e^{\prime} \in \mathrm{D}_{n, 0} \\ \operatorname{dist}\left(e^{\prime}, e\right)=1}}\left[u(e)-u\left(e^{\prime}\right)\right] \geqq 0,
$$

it follows that $u(e)=u\left(e^{\prime}\right)$ for all $e^{\prime}$ with $\operatorname{dist}\left(e, e^{\prime}\right)=1$. Iterating, and using the fact that $\mathrm{D}_{n, 0}$ is finite, we find that $\min _{e \in \mathrm{D}_{n, 0}} u(e)=\min _{e \in \operatorname{Ext}\left(\mathrm{D}_{n, 0}\right)} u(e)$, as required.

\subsection{Green's Functions in the Full Lattice}

We next assert the following lemma, concerning the existence of a full lattice Green's function $G^{\mathrm{L}}$.

Lemma 4.3. Suppose that $\mathrm{L}=\mathrm{Sq}$, Tr, or $\mathrm{Hx}$. Then there exists a lattice Green's function $G^{\mathrm{L}} \in \mathscr{W}\left(\mathrm{L}_{0}\right)$ such that

$$
G^{\mathrm{L}}(0)=0, \quad \Delta G^{\mathrm{L}}=\mathbb{1}_{0} .
$$

In addition:

(1) $G^{\mathrm{L}}$ is invariant under the group of lattice point symmetries, i.e. if $\mathrm{R}: \mathbb{R}^{2} \rightarrow \mathbb{R}^{2}$ is an orthogonal linear transformation such that $\mathrm{RL}_{0}=\mathrm{L}_{0}$, then

$$
G^{\mathrm{L}}(\mathrm{R} e)=G^{\mathrm{L}}(e) .
$$

(2) $\sup \left|\mathbf{d} G^{\mathrm{L}}(e)\right|=\mathcal{K}^{-1}$, where $\mathcal{K}$ is defined in (2.5).

$e \in \mathrm{L}_{1}$

(3) There exists a constant $C^{\mathrm{L}} \in \mathbb{R}$ so that if $u(e):=G^{\mathrm{L}}(e)+C^{\mathrm{L}}+\frac{1}{\mathcal{V}_{\pi}} \log |\operatorname{dist}(0, e)|$ for $e \in \mathrm{L} \backslash\{0\}$, then

$$
\begin{aligned}
|u(e)| & \lesssim|\operatorname{dist}(e, 0)|^{-1} \log |\operatorname{dist}(e, 0)| \text { and } \\
|\mathbf{d} u(e)| & \lesssim|\operatorname{dist}(e, 0)|^{-2} \log |\operatorname{dist}(e, 0)| .
\end{aligned}
$$

The usual method of constructing $G^{\mathrm{L}}$ is via the Fourier transform, and the existence of such a Green's function in the case of Bravais lattices is a classical assertion, as is the symmetry asserted in (1). The bounds (4.1) and (4.2) are proved in Theorem 3.5 of [17] for all Bravais lattices, thus covering the cases where $L=S q$ and $L=T r$. It therefore remains to prove (2) and the other results in the $L=H x$ case: the main observation used here is that $\mathrm{Hx}$ may be viewed as a subset of $\mathrm{Tr}$. 
Proof. We first prove (2) for $L=S q$ and $L=T r$. Fix a to be a nearest neighbour direction in the lattice. By the symmetry of $G^{\mathrm{L}}$ from (1), we have

$$
1=\Delta G^{\mathrm{L}}(0)=\mathcal{K} G^{\mathrm{L}}(0)-\mathcal{K} G^{\mathrm{L}}(0+\mathrm{a})=-\mathcal{K} G^{\mathrm{L}}(0+\mathrm{a}) .
$$

Hence $\mathbf{d} G^{\mathrm{L}}([0,0+\mathrm{a}])=\mathcal{K}^{-1}$. Now consider $v \in \mathscr{W}\left(\mathrm{L}_{0}\right)$ defined to be

$$
v(e):=G^{\mathrm{L}}(e+\mathrm{a})-G^{\mathrm{L}}(e) .
$$

It follows that $\boldsymbol{\Delta} v=\mathbb{1}_{0-\mathrm{a}}-\mathbb{1}_{0}$. Applying Lemma 4.2 on the lattice subcomplex induced by the set

$$
B_{r}^{\prime}:=\left\{e \in \mathrm{L}_{0} \mid \operatorname{dist}(e, 0) \leqq r, e \neq 0,0-\mathrm{a}\right\}
$$

we note that the maximum and minimum of $v$ are attained on $\operatorname{Ext}\left(B_{r, 0}^{\prime}\right)$, since $G^{\mathrm{L}}$ is harmonic on $\operatorname{Int}\left(B_{r, 0}^{\prime}\right)$. Now, applying (3) and letting $r$ tend to infinity implies the desired result, noting that $v(0)=-v(0-\mathrm{a})=\mathcal{K}^{-1}$.

It remains to prove the theorem for the case where $L=H x$. Recall from Section 2.3 that $\mathrm{Hx}$ may be written as

$$
H x=\sqrt{3} R_{4} \operatorname{Tr} \cup\left(\sqrt{3} R_{4} \operatorname{Tr}+e_{1}\right),
$$

and define $G^{\mathrm{Hx}} \in \mathscr{W}\left(\mathrm{Hx}_{0}\right)$ to be

$$
G^{\mathrm{Hx}}(e):=\left\{\begin{array}{c}
3 G^{\operatorname{Tr}}\left(\mathrm{R}_{4}^{T} e / \sqrt{3}\right) e \in \sqrt{3} \mathrm{R}_{4} \mathrm{Tr}, \\
\sum_{e^{\prime} \mid \operatorname{dist}\left(e^{\prime}, e\right)=1} G^{\mathrm{Hx}}\left(e^{\prime}\right) e \in \sqrt{3} \mathrm{R}_{4} \mathrm{Tr}+\mathrm{e}_{1},
\end{array}\right.
$$

where $G^{\operatorname{Tr}}$ is the lattice Green's function for $\mathrm{L}=\operatorname{Tr}$. We note that $\Delta G^{\mathrm{Hx}}(e)=0$ by definition for $e \in \sqrt{3} \mathrm{R}_{4} \mathrm{Tr}+\mathrm{e}_{1}$, and for $e \in \sqrt{3} \mathrm{R}_{4} \mathrm{Tr}$,

$$
\begin{aligned}
\Delta G^{\mathrm{Hx}}(e) & =9 G^{\operatorname{Tr}}(e / \sqrt{3})-\sum_{e^{\prime} \mid \operatorname{dist}\left(e^{\prime}, e\right)=1}\left[\sum_{e^{\prime \prime} \mid \operatorname{dist}\left(e^{\prime \prime}, e^{\prime}\right)=1} G^{\mathrm{Hx}}\left(e^{\prime \prime}\right)\right], \\
& =6 G^{\operatorname{Tr}}(e / \sqrt{3})-\sum_{e^{\prime} \mid \operatorname{dist}\left(e^{\prime}, e\right)=\sqrt{3}} G^{\operatorname{Tr}}\left(e^{\prime} / \sqrt{3}\right), \\
& =\mathbb{1}_{0}(e) .
\end{aligned}
$$

Moreover $G^{\mathrm{Hx}}(0)=G^{\operatorname{Tr}}(0)=0$, and the symmetry of $G^{\operatorname{Tr}}$ also implies (1) for $G^{\mathrm{Hx}}$.

Let $C^{\mathrm{Tr}}$ be the constant in statement (3) for the case where $\mathrm{L}=\mathrm{Tr}$, and for $e \in \mathrm{Hx} \backslash\{0\}$, define

$$
\begin{aligned}
u^{\mathrm{Hx}}(e):= & G^{\mathrm{Hx}}(e)+\frac{1}{2 \pi} \log |\operatorname{dist}(e / \sqrt{3}, 0)|+3 C^{\operatorname{Tr}}=G^{\mathrm{Hx}}(e) \\
& +\frac{1}{2 \pi} \log |\operatorname{dist}(e, 0)|+3 C^{\mathrm{Tr}}-\frac{1}{4 \pi} \log (3) .
\end{aligned}
$$


we see that for $e \in \sqrt{3} \mathrm{R}_{4} \mathrm{Tr}, u^{\mathrm{Hx}}$ satisfies (4.1) by the assertion for the case where $\mathrm{L}=\operatorname{Tr}$. For $e \in \sqrt{3} \mathrm{R}_{4} \operatorname{Tr} \backslash\{0\}$, define $v^{\operatorname{Tr}}(e):=G^{\operatorname{Tr}}(e / \sqrt{3})+\frac{1}{6 \pi} \log |\operatorname{dist}(e / \sqrt{3}, 0)|$; then for $e \in \sqrt{3} \mathrm{R}_{4} \mathrm{Tr}+\mathrm{e}_{1}$, we have

$$
u^{\mathrm{Hx}}(e)=\frac{1}{2 \pi} \log |\operatorname{dist}(e / \sqrt{3}, 0)|+\sum_{e^{\prime} \mid \operatorname{dist}\left(e, e^{\prime}\right)=1}\left(v^{\operatorname{Tr}}\left(e^{\prime}\right)-\frac{1}{6 \pi} \log \left|\operatorname{dist}\left(e^{\prime} / \sqrt{3}, 0\right)\right|\right) .
$$

Since $\log |x|$ is harmonic away from 0 , Taylor expanding to third-order about the point $e$ and using the symmetry of $\mathrm{Hx}$ implies that

$$
\frac{1}{2 \pi} \log |\operatorname{dist}(e / \sqrt{3}, 0)|-\sum_{e^{\prime} \mid \operatorname{dist}\left(e, e^{\prime}\right)=1} \frac{1}{6 \pi} \log \left|\operatorname{dist}\left(e^{\prime} / \sqrt{3}, 0\right)\right| \lesssim|\operatorname{dist}(e, 0)|^{-3} .
$$

Applying this estimate and (4.1) for $L=T r$, we obtain that

$$
|u(e)| \lesssim|\operatorname{dist}(e, 0)|^{-1} \log |\operatorname{dist}(e, 0)|
$$

for all $e \in \mathrm{Hx} \backslash\{0\}$.

To demonstrate (4.2), suppose without loss of generality that $e \in \sqrt{3} \mathrm{R}_{4} \mathrm{Tr}$ and $e+\mathrm{a} \in \sqrt{3} \mathrm{R}_{4} \mathrm{Tr}+\mathrm{e}_{1}$ for some nearest neighbour direction a. Recalling the definition of $\mathrm{a}_{i}$ from Section 2.3, for some $i$, we have

$$
\begin{aligned}
\left|\mathbf{d} u^{\mathrm{Hx}}([e, e+\mathrm{a}])\right|= & \left|v^{\operatorname{Tr}}\left(e+\sqrt{3} \mathrm{a}_{i+1}\right)+v^{\operatorname{Tr}}\left(e+\sqrt{3} \mathrm{a}_{i}\right)-2 v^{\operatorname{Tr}}(e)\right| \\
& +\frac{1}{2 \pi} \log |\operatorname{dist}(e+\mathrm{a}, 0) / \sqrt{3}| \\
& -\sum_{e^{\prime} \mid \operatorname{dist}\left(e+\mathrm{a}, e^{\prime}\right)=1} \frac{1}{6 \pi} \log \left|\operatorname{dist}\left(e^{\prime}, 0\right) / \sqrt{3}\right| .
\end{aligned}
$$

Using the definition of $v^{\operatorname{Tr}}$, and then applying statement (3) in the case $\mathrm{L}=\operatorname{Tr}$ as well as (4.3) gives the result.

\subsection{The Harmonic Measure and Interior Differential Estimates}

We now define the harmonic measure, which allows us to express functions which are harmonic in a region in terms of their boundary values. In order to do so, we introduce $Q^{r}$, which should be thought of as 'balls of radius $r$ ' in the lattice, and are defined to be:

$Q^{r}:= \begin{cases}{[-r, r]^{2} \cap \mathrm{L}} & \mathrm{L}=\mathrm{Sq}, \\ \left\{x \in \mathbb{R}^{2}|| x \cdot\left(\mathrm{a}_{1}+\mathrm{a}_{2}\right)|,| x \cdot\left(\mathrm{a}_{2}+\mathrm{a}_{3}\right)|,| x \cdot\left(\mathrm{a}_{3}+\mathrm{a}_{4}\right) \mid \leqq \frac{1}{2} r\right\} \cap \mathrm{L} \mathrm{L}=\mathrm{Tr}, \mathrm{Hx} .\end{cases}$

Lemma 4.4. Let $Q^{r}$ be as defined in (4.4). Then for each e $\in \operatorname{Ext}\left(Q_{0}^{r}\right)$, there exists $\left.\omega_{e}^{r} \in \mathscr{W}\left(Q_{0}^{r}\right)\right)$ satisfying

$$
\boldsymbol{\Delta} \omega_{e}^{r}=0 \text { in } \operatorname{Int}\left(Q_{0}^{r}\right), \text { with } \omega_{e}^{r}=\frac{\mathbb{1}_{e}}{\# \operatorname{Ext}\left(Q_{0}^{r}\right)} \text { on } \operatorname{Ext}\left(Q_{0}^{r}\right) .
$$

In addition, $\omega_{e}^{r}$ satisfies the following properties: 
(1) If $u \in \mathscr{W}\left(Q_{0}^{r}\right)$ is harmonic in $Q_{0}^{r}$, then for any $e^{\prime} \in \mathrm{D}_{n, 0}$,

$$
u\left(e^{\prime}\right)=\sum_{e \in \operatorname{Ext}\left(Q_{0}^{r}\right)} \omega_{e}^{r}\left(e^{\prime}\right) u(e)
$$

(2) There exists a constant $C^{\mathrm{L}}>0$ depending only on $\mathrm{L}$ such that

$$
\left|\mathbf{d} \omega_{e}^{r}([0,0+\mathrm{a}])\right| \leqq C^{\mathrm{L}} \log (r) r^{-2}
$$

for any nearest-neighbour direction a.

The function $\omega_{e}^{r}$ is called the harmonic measure, and enjoys a variety of interpretations, both probabilistic and and analytic: for further detail, we refer the reader to [23]. Its principal use will be as a tool by which we can estimate the effect of the boundary conditions on the solution in the domain interior.

The existence of $\omega_{e}^{r}$ and statement (1) follow directly from Lemma 4.1. In the case where $\mathrm{L}=\mathrm{Sq}$, a proof of (4.5) with the improved upper bound $C^{\mathrm{L}} r^{-2}$ is given in Lemma 3 of [26] using an explicit construction of $\omega_{e}^{r}$. Further results on the harmonic measure in the square lattice may also be found in Chapter 8 of [35].

Proof. It remains to prove (2). We use the discrete analogue of Green's formula:

$$
\int_{\operatorname{Int}\left(Q_{0}^{r}\right)} u \boldsymbol{\Delta} v \boldsymbol{\Delta} u=\int_{\operatorname{Ext}\left(Q_{0}^{r}\right)} u \boldsymbol{\Delta}-v \boldsymbol{\Delta} u=\sum_{e \in \operatorname{Ext}\left(Q_{0}^{r}\right)}\left[u(e)\left(\int_{\delta e} \mathbf{d} v\right)-v(e)\left(\int_{\delta e} \mathbf{d} v\right)\right],
$$

which follows by applying (2.1) to the extension of $u, v, \mathbf{d} u$ and $\mathbf{d} v$ by 0 to the full lattice complex. Now, consider $v$ which is the solution to

$$
\Delta v=0, \quad v(e)=G^{\mathrm{L}}(e+\mathrm{a})-G^{\mathrm{L}}(e) \text { on } \operatorname{Ext}\left(Q_{0}^{r}\right) .
$$

Such $v$ clearly exists by Lemma 4.1, and by applying Lemma 4.2 and then Lemma 4.3, we obtain that

$$
\sup _{e^{\prime} \in Q_{1}^{r}}\left|\mathbf{d} v\left(e^{\prime}\right)\right| \leqq 2 \sup _{e^{\prime} \in Q_{0}^{r}}\left|v\left(e^{\prime}\right)\right| \leqq 2 \sup _{e^{\prime} \in \operatorname{Ext}\left(Q_{0}^{r}\right)}\left|v\left(e^{\prime}\right)\right| \leqq C^{\mathrm{L}} r^{-1} \log (r) .
$$

Defining $u \in \mathscr{W}\left(Q_{0}^{r}\right)$ to be $u(e):=G^{\mathrm{L}}(e+\mathrm{a})-G^{\mathrm{L}}(e)-v(e), u$ vanishes on $\operatorname{Ext}\left(Q_{0}^{r}\right)$, and we have that $\Delta u=\mathbb{1}_{e+a}-\mathbb{1}_{e}$, and hence

$$
\mathbf{d} \omega_{e}^{r}([0,0+\mathrm{a}])=\int_{\operatorname{Int}\left(Q_{0}^{r}\right)} \omega_{r}^{r} \boldsymbol{\Delta} u-u \boldsymbol{\Delta} \omega_{e}^{r}=\int_{\operatorname{Ext}\left(Q_{0}^{r}\right)} \omega_{e}^{r} \boldsymbol{\Delta} u-u \boldsymbol{\Delta} \omega_{e}^{r}=\frac{\boldsymbol{\Delta} u(e)}{\# \operatorname{Ext}\left(Q_{0}^{r}\right)} .
$$

Now, applying (4.2) and (4.6), we obtain

$$
|\Delta u(e)| \leqq C^{\mathrm{L}} r^{-2} \log (r)
$$

which completes the proof.

The harmonic measure now allows us to obtain the following interior bound on the differential of a harmonic lattice form $u$. 
Lemma 4.5. Suppose that $u \in \mathscr{W}\left(\mathrm{D}_{n, 0}\right)$ satisfies $\Delta u=0$ and and $u=g$ on $\operatorname{Ext}\left(\mathrm{D}_{n, 0}\right)$ with $g: \operatorname{Ext}\left(\mathrm{D}_{n, 0}\right) \rightarrow \mathbb{R}$. Then there exists a constant $C^{\mathrm{L}}>0$ depending only on $\mathrm{L}$ such that

$$
|\mathbf{d} u(e)| \leqq C^{\mathrm{L}} \frac{\log \left(\operatorname{dist}\left(e, \operatorname{Ext}\left(\mathrm{D}_{n, 0}\right)\right)\right.}{\operatorname{dist}\left(e, \operatorname{Ext}\left(\mathrm{D}_{n, 0}\right)\right)} \sup _{e^{\prime} \in \operatorname{Ext}\left(\mathrm{D}_{n, 0}\right)}\left|g\left(e^{\prime}\right)\right| \quad \text { for any } e \in \mathrm{D}_{n, 1} .
$$

Proof. Suppose that $e=\left[e_{0}, e_{1}\right] \in \mathrm{D}_{n, 1}$, and let $\mathrm{x} \in \mathrm{D}$ be the vector corresponding to $e_{0}$. Let $Q^{r}$ be as defined in (4.4), where $r=\left\lfloor\operatorname{dist}\left(e_{0}, \operatorname{Ext}\left(\mathrm{D}_{n, 0}\right)\right)\right\rfloor$; then $\mathrm{x}+Q_{0}^{r} \subset$ $\mathrm{D}_{n, 0}$, and statement (1) in Lemma 4.4 implies that

$$
w(e)=\sum_{e^{\prime} \in \operatorname{Ext}\left(\mathbf{x}+Q_{0}^{r}\right)} \omega_{e^{\prime}}^{r}(e) w\left(e^{\prime}\right), \quad \text { so } \mathbf{d} w(e)=\sum_{e^{\prime} \in \operatorname{Ext}\left(\mathbf{a}+Q_{0}^{r}\right)} \mathbf{d} \omega_{e^{\prime}}^{r}(e) w\left(e^{\prime}\right) .
$$

Applying statement (2) of Lemma 4.4 and Lemma 4.2, it follows that

$$
|\mathbf{d} w(e)| \leqq \sup _{e^{\prime} \in \operatorname{Ext}\left(\mathbf{x}+Q_{0}^{r}\right)}\left|w\left(e^{\prime}\right)\right| \sum_{e^{\prime} \in \operatorname{Ext}\left(\mathbf{x}+Q_{0}^{r}\right)}\left|\mathbf{d} \omega_{e^{\prime}}^{r}(e)\right| \leqq C^{\mathrm{L}} \frac{\log (r)}{r} \sup _{e^{\prime} \in \operatorname{Ext}\left(\mathrm{D}_{n, 0}\right)}\left|g\left(e^{\prime}\right)\right| .
$$

\subsection{Asymptotics for Green's Functions on Finite Subcomplexes}

We have now collected the necessary analytical tools with which we will prove Theorem 3.1: our final auxiliary result is the following precise description of the differential of solutions to (3.2).

Theorem 4.6. Suppose that $\mu=\sum_{i=1}^{m} b_{i} \mathbb{1}_{e_{i}} \in \mathscr{M}_{n}^{\varepsilon}$, and let $G_{\mu^{*}} \in \mathscr{W}\left(\mathrm{D}_{n, 0}^{*}\right)$ be the solution to (3.2). Let $e \in \mathrm{D}_{n, 0}^{*}$ with $[e, e+\mathrm{a}] \in \mathrm{D}_{1}^{*}$, and let $x \in \mathbb{R}^{2}$ correspond to the dual 0 -cell e; then we have

$$
\begin{aligned}
\mathbf{d}^{*} G_{\mu^{*}}([e, e+\mathrm{a}])= & b_{i} \mathbf{d}^{*} G^{\mathrm{L}^{*}}\left(\left[e-\mathbf{x}_{i}, e+\mathrm{a}-\mathbf{x}_{i}\right]\right) \\
& +n^{-1}\left[b_{i} \nabla \bar{y}_{i}\left(\frac{1}{n} x\right) \cdot \mathrm{a}+\sum_{j \neq i} b_{j} \nabla \mathcal{G}_{\mathbf{x}_{j}}\left(\frac{1}{n} x\right) \cdot \mathrm{a}\right] \\
& +O\left(n^{-1-\delta} \log (n)\right),
\end{aligned}
$$

where:

(1) $G^{\mathrm{L}^{*}}$ is the full lattice Green's function for $\mathrm{L}^{*}$, whose existence was asserted in Theorem 4.3,

(2) $e_{i}^{*}$ minimises $\operatorname{dist}\left(x, e_{i}^{*}\right)$ over all $i=1, \ldots, m$,

(3) for each $i, \mathbf{x}_{i} \in \mathrm{D}$ satisfies $\operatorname{dist}\left(\mathbf{x}_{i}, \frac{1}{n} e_{i}^{*}\right) \leqq \frac{1}{n}$,

(4) $\mathcal{G}_{y}$ is the continuum Dirichlet Green's function on D corresponding to the point $y$, i.e. the solution to

$$
-\Delta \mathcal{G}_{y}(\cdot)=\frac{\mathcal{V}}{2} \delta(\cdot-y) \text { in } \mathrm{D}, \text { with } \mathcal{G}_{y}=0 \text { on } \partial \mathrm{D}
$$


(5) $\bar{y}_{i}$ solves

$$
-\Delta \bar{y}_{i}=0 \text { in } \mathrm{D}, \quad \text { with } \bar{y}_{i}(s)=\frac{1}{\mathcal{V} \pi} \log \left(\left|s-x_{i}\right|\right) \text { on } \partial \mathrm{D},
$$

(6) $\delta>0$ is an exponent which depends only on $\varphi_{l}$, the interior angles at the corners of the lattice polygon $\mathrm{D}$, and

(7) $O\left(n^{-1-\delta} \log (n)\right)$ denotes an error term which is uniform for all $\mu \in \mathscr{M}_{n}^{\varepsilon}$.

The proof of this result is technical, so we first outline the main strategy, which is similar in spirit to the approach taken in the proof of Theorem 3.3 in [31]. We decompose $G_{\mu^{*}}$ as a sum of

(1) full lattice Green's functions restricted to $D_{n, 0}^{*}$,

(2) continuum boundary correctors $\bar{y}_{i}$, and

(3) discrete correctors.

Each of these components are treated separately, applying Lemma 4.3, the regularity theory of [25], the maximum principle proved in Lemma 4.2 and the interior estimate of Lemma 4.5 to analyse each piece. Since the entire proof takes place in the dual complex $\mathrm{D}_{n}^{*}$, for brevity we drop $*$ from our notation throughout.

Decomposition of $G_{\mu}$ For $i=1, \ldots, m$, let $\mathrm{x}_{i} \in \mathbb{R}^{2}$ be the vector corresponding to the point $e_{i}$. We begin by decomposing

$$
G_{\mu}(e)=\sum_{i=1}^{m} b_{i} G^{\mathrm{L}}\left(e-\mathbf{x}_{i}\right)+\sum_{i=1}^{m} \bar{y}_{i}^{n}(e)+u(e)+v(e),
$$

where:

(1) $G^{\mathrm{L}}$ is the full (dual) lattice Green's function,

(2) $\bar{y}_{i}^{n}(x):=\bar{y}_{i}\left(\frac{1}{n} x\right)$, where $\bar{y}_{i}$ solves $\Delta \bar{y}_{i}=0$ on $\mathrm{D}$ with boundary values

$$
\bar{y}_{i}(x)=\frac{1}{\mathcal{V} \pi} b_{i} \log \left(\left|x-\mathbf{x}_{i}\right|\right),
$$

(3) $u$ is the solution to the discrete Poisson problem

$$
\Delta u=0 \quad \text { with } u(e)=-\sum_{i=1}^{m}\left[b_{i} G^{\mathrm{L}}\left(e-\mathrm{x}_{i}\right)+\bar{y}_{i}^{n}(e)\right] \text { on } \operatorname{Ext}\left(\mathrm{D}_{n, 0}\right) \text {, and }
$$

(4) $v$ is the solution to the discrete Poisson problem

$$
\Delta v=-\sum_{i=1}^{m} \boldsymbol{\Delta} \bar{y}_{i}^{n} \quad \text { with } \quad v(e)=0 \text { on } \operatorname{Ext}\left(\mathrm{D}_{n, 0}\right)
$$

In combination, Lemma 4.3, the theory of boundary value problems on polygons in [25], and Lemma 4.1 allow us to conclude that each of the terms in this decomposition is well-defined. Furthermore, equality follows since solutions to the Poisson problem are unique by Lemma 4.1. 
Regularity of $\bar{y}_{i} \quad$ We now recall some facts concerning the regularity of $\bar{y}_{j}$ from [25]. Applying Theorem 6.4.2.6 in [25], there exists $\sigma \in(0,1)$ such that $\bar{y}_{i}$ lies in the space

$$
\mathcal{X}:=\mathrm{C}^{4, \sigma}(\mathrm{D})+\operatorname{span}\left\{\mathfrak{S}_{l, m} \mid m \in \mathbb{N}, 0<m<(4+\sigma) \varphi_{l} / \pi\right\},
$$

where $\mathfrak{S}_{l, m}$ is given in polar coordinates $\left(r_{l}, \theta_{l}\right)$ about the $c_{l}$, the $l$ th corner of $\mathrm{D}$ as

$$
\mathfrak{S}_{l, m}\left(r_{l}, \theta_{l}\right):= \begin{cases}r_{l}^{m \pi / \varphi_{l}} \sin \left(\frac{m \pi}{\varphi_{l}} \theta_{l}\right) \eta\left(r_{l}\right), & m \pi / \varphi_{l} \notin \mathbb{N}, \\ r_{l}^{m \pi / \varphi_{l}} \log \left(r_{l}\right)\left[\sin \left(\frac{m \pi}{\varphi_{l}} \theta_{l}\right)+\theta_{l} \cos \left(\frac{m \pi}{\varphi_{l}} \theta_{l}\right)\right] \eta\left(r_{l}\right), & m \pi / \varphi_{l} \in \mathbb{N} .\end{cases}
$$

We recall that $\varphi_{l}$ is the interior angle at $c_{l}$, and we set $\eta \in \mathrm{C}_{0}^{\infty}(\mathbb{R})$ to be a cutoff function so that $\eta(x)=1$ for $|x|$ sufficiently small, and $\operatorname{supp}\left\{\mathfrak{S}_{l, m}\right\} \cap \operatorname{supp}\left\{\mathfrak{S}_{l^{\prime}, n}\right\}=$ $\emptyset$ for any $n, m \in \mathbb{N}$ and any $l \neq l^{\prime}$.

We note that $\bar{y}_{i}$ only fails to be $\mathrm{C}^{4, \sigma}$ at the corners of the domain $\mathrm{D}$, and since $\mathrm{D}$ is convex, $\pi / \varphi_{l}>1$, which implies that $\mathfrak{S}_{l, m} \in \mathrm{C}^{1, \delta}(\mathrm{D})$. Hence $\bar{y}_{i} \in \mathrm{C}^{1, \delta}(\mathrm{D})$ with $\delta:=\min _{l}\left\{\pi / \varphi_{l}-1\right\} \in\left(0, \frac{1}{2}\right]$. $\mathcal{X}$ is a Banach space when endowed with the norm

$$
\left\|\underset{0<m<(4+\sigma) \varphi_{l} / \pi}{v+} C_{l, m} \mathfrak{S}_{l, m}\right\|_{\mathcal{X}}:=\|v\|_{\mathrm{C}^{4, \sigma}(\mathrm{D})}+\sum_{0<m<(4+\sigma) \varphi_{l} / \pi}\left|C_{l, m}\right| .
$$

Furthermore, it can be checked that the mapping

$$
\mathcal{S}:\{x \in \mathrm{D} \mid \operatorname{dist}(x, \partial \mathrm{D}) \geqq \varepsilon / 2\} \rightarrow \mathcal{X} \text { where } \mathcal{S}\left(x_{i}\right):=\bar{y}_{i}
$$

is continuous, and is hence bounded, since the domain of $\mathcal{S}$ is compact.

Estimating $\mathbf{d} G^{\mathrm{L}}$ and $\mathbf{d} \bar{y}_{j}^{n} \quad$ Applying (4.2) for any $e_{j}$ which is not the closest point to $e$ in the support of $\mu$, we have that

$$
\begin{aligned}
& \left|\mathbf{d} G^{\mathrm{L}}\left(e-\mathbf{x}_{j}, e+\mathrm{a}-\mathbf{x}_{j}\right)-\int_{0}^{1} \frac{1}{\mathcal{V} \pi} \frac{e+t \mathbf{a}-\mathbf{x}_{j}}{\left|e+t \mathbf{a}-\mathbf{x}_{j}\right|^{2}} \cdot \mathrm{ad} t\right| \lesssim \frac{\log \left|\operatorname{dist}\left(e-\mathbf{x}_{j}, 0\right)\right|}{\operatorname{dist}\left(e-\mathbf{x}_{j}, 0\right)^{2}} \\
& \quad=O\left(n^{-2} \log (n)\right),
\end{aligned}
$$

which holds uniformly for $\mu \in \mathscr{M}_{n}^{\varepsilon} \operatorname{since} \operatorname{dist}\left(e, e_{j}\right) \geqq \frac{1}{2} \operatorname{dist}\left(e_{i}, e_{j}\right) \geqq \frac{1}{2} \varepsilon n$. Furthermore, using the homogeneity and regularity of $(x, y) \mapsto \frac{x-y}{|x-y|^{2}}$ to Taylor expand under the integral, we have

$$
\mathbf{d} G^{\mathrm{L}}\left(\left[e-\mathbf{x}_{j}, e+\mathrm{a}-\mathbf{x}_{j}\right]\right)=n^{-1} \frac{1}{\mathcal{K} \pi} \frac{\frac{1}{n} e-\mathbf{x}_{j}}{\left|\frac{1}{n} e-\mathbf{x}_{j}\right|^{2}} \cdot \mathbf{a}+O\left(n^{-2} \log (n)\right) .
$$

Using the representation of $\bar{y}_{j} \in \mathcal{X}$ and Taylor expanding, we have

$$
\left|\mathbf{d} \bar{y}_{j}^{n}([e, e+\mathrm{a}])-n^{-1} \nabla \bar{y}_{j}\left(\frac{1}{n} e\right) \cdot \mathrm{a}\right| \leqq n^{-2} \sum_{l=1}^{L}\left|\operatorname{dist}\left(\frac{1}{n} e, c_{l}\right)\right|^{\pi / \varphi_{l}-2}\left\|\bar{y}_{j}\right\| \mathcal{X} .
$$


Estimating $\mathbf{d} u$ We now use Lemma 4.5 to estimate $\mathbf{d} u$. Define $g: \operatorname{Ext}\left(\mathrm{D}_{n, 0}\right) \rightarrow \mathbb{R}$ to be

$$
g(e):=\sum_{j=1}^{m} b_{j} G^{\mathrm{L}}\left(e-\mathbf{x}_{j}\right)+\bar{y}_{j}^{n}(e) .
$$

By applying (4.1) and again invoking the definition and regularity of $\bar{y}_{j}^{n}$ to Taylor expand near the boundary, we have that

$$
\|g\|_{\ell^{\infty}\left(\operatorname{Ext}\left(\mathrm{D}_{n, 0}\right)\right)} \lesssim n^{-1}\|\bar{y}\|_{\mathcal{X}}+O\left(n^{-1} \log (n)\right),
$$

where the latter term is uniform in $n$ for fixed $\varepsilon$. Lemma 4.5 now implies that

$$
|\mathbf{d} u(e)| \lesssim n^{-1} \log (n)\left|\operatorname{dist}\left(e, \operatorname{Ext}\left(\mathrm{D}_{n, 0}\right)\right)\right|^{-1} .
$$

Estimating $\boldsymbol{\Delta} \bar{y}_{j}^{n} \quad$ For the purpose of estimating $\mathbf{d} v$, we first obtain bounds on $\Delta \bar{y}_{j}^{n}$. Let $e \in \mathrm{D}_{n, 0} \backslash \operatorname{Ext}\left(\mathrm{D}_{n, 0}\right)$, and $x \in \mathrm{D}$ be the corresponding vector. We use the regularity of $\bar{y}_{j}$ to Taylor expand, obtaining

$$
\begin{aligned}
\Delta \bar{y}_{j}^{n}(e)= & \sum_{j=1}^{m} \sum_{i=1}^{\mathcal{K}} \int_{0}^{1} \nabla \bar{y}_{j}\left(\frac{1}{n}\left(x+t \mathrm{~s}_{i}\right)\right) \cdot \frac{1}{n} \mathrm{~s}_{i} \mathrm{~d} t \\
= & \sum_{j=1}^{m} \sum_{i=1}^{\mathcal{K}} \int_{0}^{1} \frac{1}{2} n^{-3} \nabla^{3} \bar{y}_{j}\left(\frac{1}{n} x\right)\left[\mathrm{s}_{i}, \mathrm{~s}_{i}, \mathrm{~s}_{i}\right] \\
& +\frac{1}{6} n^{-4}(1-t)^{3} \nabla^{4} \bar{y}_{j}\left(\frac{1}{n}\left(x+t \mathrm{~s}_{i}\right)\right)\left[\mathrm{s}_{i}, \mathrm{~s}_{i}, \mathrm{~s}_{i}, \mathrm{~s}_{i}\right] \mathrm{d} t
\end{aligned}
$$

where $\mathrm{s}_{i}$ are nearest neighbour directions in the dual lattice, and the terms involving $\nabla \bar{y}_{j}$ and $\nabla^{2} \bar{y}_{j}$ cancel respectively by lattice symmetry and the fact that $\bar{y}_{j}$ is harmonic. If the dual lattice is Sq or $\mathrm{Tr}$, then the terms involving $\nabla^{3} \bar{y}_{j}$ also cancel, which entails that

$$
\left|\boldsymbol{\Delta} \bar{y}_{j}^{n}(e)\right| \leqq \frac{1}{6} n^{-4} \sum_{i=1}^{m} \int_{0}^{1}(1-t)^{3}\left|\nabla^{4} \bar{y}_{j}\left(\frac{1}{n}\left(x+t \mathrm{~s}_{i}\right)\right)\right| \mathrm{d} t .
$$

By using the description of $\bar{y}_{j}$ as a sum of $v \in \mathrm{C}^{4, \sigma}(\mathrm{D})$ and $\mathfrak{S}_{j, m}$, it can be seen that each of the integrands in the estimate above is bounded any $e \in \mathrm{D}_{n, 0}$ and $\mathrm{s}_{i}$, and moreover

$$
\left|\boldsymbol{\Delta} \bar{y}_{j}^{n}(e)\right| \leqq \frac{1}{6} \mathcal{K} n^{-4}\|\bar{y}\|_{\mathcal{X}} \sum_{l}\left|\operatorname{dist}\left(\frac{1}{n} e, c_{l}\right)\right|^{\pi / \varphi_{l}-4} .
$$

Returning to the case where the dual lattice is $\mathrm{Hx}$, we first Taylor expand to third-order to obtain that

$$
\left|\boldsymbol{\Delta} \bar{y}_{j}^{n}(e)\right| \leqq \frac{1}{2} n^{-3} \sum_{l} \operatorname{dist}\left(\frac{1}{n} e, c_{l}\right)^{\pi / \varphi_{l}-3}\|\bar{y}\| \mathcal{X} .
$$

Define

$$
A:=\left\{e \in \operatorname{Int}\left(\mathrm{D}_{n, 0}\right) \mid e, e+\mathrm{e}_{1} \in \operatorname{Int}\left(\mathrm{D}_{n, 0}\right)\right\}
$$


then for all $e \in A$, we have

$$
\begin{aligned}
& \left|\frac{1}{2} n^{-3} \sum_{i=1}^{3}\left(\nabla^{3} \bar{y}_{j}\left(\frac{1}{n} e\right)\left[\mathrm{a}_{2 i}, \mathrm{a}_{2 i}, \mathrm{a}_{2 i}\right]-\nabla^{3} \bar{y}_{j}\left(\frac{1}{n}\left(e+\mathrm{e}_{1}\right)\right)\left[\mathrm{a}_{2 i}, \mathrm{a}_{2 i}, \mathrm{a}_{2 i}\right]\right)\right| \\
& \quad=\left|\frac{1}{2} n^{-4} \sum_{i=1}^{3} \int_{0}^{1} \nabla^{4} \bar{y}_{j}\left(\frac{1}{n}\left(e+t \mathrm{e}_{1}\right)\right)\left[\mathrm{e}_{1}, \mathrm{a}_{i}, \mathrm{a}_{i}, \mathrm{a}_{i}\right]\right| \\
& \quad \leqq \frac{3}{2} n^{-4} \sum_{l} \operatorname{dist}\left(\frac{1}{n} e, c_{l}\right)^{\pi / \varphi_{l}-4}\|\bar{y}\| \mathcal{X} .
\end{aligned}
$$

Using this estimate, and the argument used above in the case where the dual lattice was $\operatorname{Tr}$, for any $e \in A$, we deduce that

$$
\left|\boldsymbol{\Delta} \bar{y}_{j}^{n}(e)+\boldsymbol{\Delta} \bar{y}_{j}^{n}\left(e+\mathrm{e}_{1}\right)\right| \leqq 2 n^{-4} \sum_{l} \operatorname{dist}\left(\frac{1}{n} e, c_{l}\right)^{\pi / \varphi_{l}-4}\|\bar{y}\|_{\mathcal{X}} .
$$

Estimating $\mathbf{d} v$ It remains to bound $\mathbf{d} v$. We proceed by constructing upper and lower bounds on $v$ by using estimates (4.14), (4.15) and (4.17) and the full lattice Green's function. Recalling the result of Lemma 4.3, for any $\mathrm{x} \in \mathrm{D}$, we note that

$$
\begin{gathered}
\Delta\left[G^{\mathrm{L}}(\cdot-x)+\frac{1}{\mathcal{V} \pi} \log |n \operatorname{diam}(\mathrm{D})|\right]=\mathbb{1}_{e} \quad \text { in } \operatorname{Int}\left(\mathrm{D}_{n, 0}\right), \text { and } \\
G^{\mathrm{L}}(\cdot-x)+\frac{1}{\mathcal{V}_{\pi}} \log |n \operatorname{diam}(\mathrm{D})| \geqq 0 \quad \text { on } \operatorname{Ext}\left(\mathrm{D}_{n, 0}\right) .
\end{gathered}
$$

Next, we define neighbourhoods of each corner of the domain

$$
B_{l, \varepsilon}:=\left\{e \in \operatorname{Int}\left(\mathrm{D}_{n, 0}\right) \mid \operatorname{dist}\left(\frac{1}{n} e, c_{l}\right) \leqq \varepsilon\right\} .
$$

Recalling that $\delta:=\min _{l}\left\{\frac{\pi}{\varphi_{l}}-1\right\} \in\left(0, \frac{1}{2}\right]$, estimate (4.14) implies that

$$
\left|\boldsymbol{\Delta} \bar{y}_{j}^{n}(e)\right| \lesssim n^{-4} \varepsilon^{\delta-3}\left\|\bar{y}_{j}\right\| \mathcal{X} \quad \text { on } \operatorname{Int}\left(\mathrm{D}_{n, 0}\right) \backslash \bigcup_{l} B_{l, \varepsilon} .
$$

We now define

$$
v^{ \pm}(e):=-\left[\sum_{e^{\prime} \in \mathrm{D}_{n, 0}} \Delta \bar{y}_{j}^{n}\left(e^{\prime}\right) G^{\mathrm{L}}\left(e-e^{\prime}\right)\right] \pm C_{n},
$$

where $C_{n}$ is a small constant depending upon $n$ that we will choose later. We note that $\boldsymbol{\Delta}\left[v-v^{ \pm}\right]=0$, so choosing $C_{n}$ such that $v^{+} \geqq 0$ and $v^{-} \leqq 0$ on $\operatorname{Ext}\left(\mathrm{D}_{n, 0}\right)$, Lemma 4.2 would imply that

$$
v^{-}(e) \leqq v(e) \leqq v^{+}(e) \text { for all } e \in \operatorname{Int}\left(\mathrm{D}_{n, 0}\right) .
$$

When the dual lattice is either Tr or Sq, applying estimate (4.18), and summing,

$$
\begin{aligned}
\left|v^{ \pm}(e)\right| \lesssim\|\bar{y}\| \mathcal{X}\left[\sum_{\substack{e^{\prime} \in \operatorname{Int}\left(\mathrm{D}_{n, 0}\right) \\
e^{\prime} \notin \bigcup_{l} B_{l, \varepsilon}}} n^{-4} \varepsilon^{\delta-3}\left|G^{\mathrm{L}}\left(e-e^{\prime}\right)\right|\right. \\
\left.+n^{-1-\delta} \sum_{e \in \bigcup_{l} B_{l, \varepsilon}} \operatorname{dist}\left(e^{\prime}, n c_{l}\right)^{\delta-3}\left|G^{\mathrm{L}}\left(e-e^{\prime}\right)\right|\right]+C_{n} n^{2} .
\end{aligned}
$$


Treating each sum separately, we see that

$$
\begin{aligned}
& \sum_{\substack{e^{\prime} \in \operatorname{Int}\left(\mathrm{D}_{n, 0}\right) \\
e^{\prime} \notin \bigcup_{l} B_{l, \varepsilon}}}\left|G^{\mathrm{L}}\left(e-e^{\prime}\right)\right| \lesssim \sum_{\substack{e^{\prime} \in \operatorname{Int}\left(\mathrm{D}_{n, 0}\right) \\
e^{\prime} \notin \bigcup_{l} B_{l, \varepsilon}}} \log \left|\operatorname{dist}\left(e, e^{\prime}\right)+1\right| \lesssim n^{2} \log (n), \\
& \sum_{e^{\prime} \in \bigcup_{l} B_{l, \varepsilon}}\left|\operatorname{dist}\left(e^{\prime}, n c_{l}\right)\right|^{\delta-3}\left|G^{\mathrm{L}}\left(e-e^{\prime}\right)\right| \lesssim \log (n) \sum_{e \in \bigcup_{l} B_{l, r}}\left|\operatorname{dist}\left(e^{\prime}, n c_{l}\right)\right|^{\delta-3} \lesssim \log (n),
\end{aligned}
$$

recalling that statement (3) of Theorem 4.3 implies that $\left|G^{\mathrm{L}}(e)\right| \lesssim \log |\operatorname{dist}(e, 0)|$, $\operatorname{diam}(n \mathrm{D})=O(n)$, and the sum on the second line converges since $\delta \leqq \frac{1}{2}<1$.

These estimates imply that

$$
\left|v^{ \pm}(e)\right| \lesssim\|\bar{y}\| \mathcal{X} n^{-1-\delta} \log (n)+C_{n} n^{2},
$$

so choosing $C_{n}=O\left(n^{-3-\delta} \log (n)\right)$ gives

$$
|v(e)|=O\left(n^{-1-\delta} \log (n)\right), \quad \text { and hence } \quad|\mathbf{d} v(e)|=O\left(n^{-1-\delta} \log (n)\right)
$$

for all $e \in \mathrm{D}_{n, 1}$.

When the dual lattice is $\mathrm{Hx}$, recall the definition of $A$ from (4.16), and set

$$
A^{\prime}:=\left\{e \in \operatorname{Int}\left(\mathrm{D}_{n, 0}\right) \mid e-\mathrm{e}_{1} \notin \operatorname{Int}\left(\mathrm{D}_{n, 0}\right)\right\}
$$

For any $e^{\prime} \in A$ let $\mathbf{x}^{\prime} \in \mathrm{D}$ be the corresponding vector. We apply (4.15), (4.17), and the conclusions of Theorem 4.3 to deduce that

$$
\begin{aligned}
& \left|G^{\mathrm{Hx}}\left(e-\mathbf{x}^{\prime}\right) \boldsymbol{\Delta} \bar{y}_{j}^{n}(e)+G^{\mathrm{Hx}}\left(e+\mathrm{e}_{1}-\mathbf{x}^{\prime}\right) \boldsymbol{\Delta} \bar{y}_{j}^{n}\left(e+\mathrm{e}_{1}\right)\right| \\
& \leqq\left|G^{\mathrm{Hx}}\left(e-\mathrm{x}^{\prime}\right)\right| n^{-4}\left|\operatorname{dist}\left(\frac{1}{n} e, c_{l}\right)\right|^{\pi / \varphi_{l}-4} \\
& +\left|\mathbf{d} G^{\mathrm{Hx}}\left(e-\mathbf{x}^{\prime}, e+\mathrm{e}_{1}-\mathbf{x}^{\prime}\right)\right| n^{-3}\left|\operatorname{dist}\left(\frac{1}{n} e, c_{l}\right)\right|^{\pi / \varphi_{l}-3} \\
& \leqq \log \left|\operatorname{dist}\left(e, \mathbf{x}^{\prime}\right)\right| n^{-4}\left|\operatorname{dist}\left(\frac{1}{n} e, c_{l}\right)\right|^{\pi / \varphi_{l}-4} \\
& +\frac{\log \left|\operatorname{dist}\left(e, \mathbf{x}^{\prime}\right)\right|}{\operatorname{dist}\left(e, \mathbf{X}^{\prime}\right)} n^{-3}\left|\operatorname{dist}\left(\frac{1}{n} e, c_{l}\right)\right|^{\pi / \varphi_{l}-3} \text {. }
\end{aligned}
$$

By summing over $e^{\prime} \in A$, we obtain

$$
\begin{aligned}
& \sum_{e^{\prime} \in A}\left|G^{\mathrm{Hx}}\left(e-\mathrm{x}^{\prime}\right) \Delta \bar{y}_{j}^{n}\left(e^{\prime}\right)+G^{\mathrm{Hx}}\left(e+\mathrm{e}_{1}-\mathrm{x}^{\prime}\right) \Delta \bar{y}_{j}^{n}\left(e^{\prime}+\mathrm{e}_{1}\right)\right| \\
& \quad \lesssim\left\|\bar{y}_{j}\right\|_{\mathcal{X}}\left[\log (n) n^{-2} \varepsilon^{\delta-3}+n^{-1-\delta} \log (n)\right] .
\end{aligned}
$$

Next, we sum (4.15) over $A^{\prime}$, noting that \# $A^{\prime}=O(n)$, to obtain 


$$
\begin{aligned}
& \sum_{e^{\prime} \in A^{\prime}}\left|G^{\mathrm{L}}\left(e-\mathbf{x}^{\prime}\right)\right|\left|\boldsymbol{\Delta} \bar{y}_{j}^{n}\left(e^{\prime}\right)\right| \\
& \quad \lesssim\left\|\bar{y}_{j}\right\| \mathcal{X} \log (n)\left[\sum_{\substack{e^{\prime} \in A^{\prime} \\
e^{\prime} \notin \bigcup_{l} B_{l, \varepsilon}}} n^{-3} \varepsilon^{\delta-2}+n^{-1-\delta} \sum_{\substack{e^{\prime} \in A^{\prime} \\
e^{\prime} \in \bigcup_{l} B_{l, \varepsilon}}} \operatorname{dist}\left(e^{\prime}, n c_{l}\right)^{\delta-2}\right] \\
& \quad \lesssim\left\|\bar{y}_{j}\right\| \mathcal{X}\left[\varepsilon^{\delta-2} \log (n) n^{-2}+n^{-1-\delta} \log (n)\right] .
\end{aligned}
$$

Putting (4.20) and (4.21) together, and applying similar arguments to that made for the other cases above, we deduce that (4.19) also holds in the case where the dual lattice is $\mathrm{Hx}$.

Conclusion Combining (4.9), (4.10), (4.12) and (4.19) and noting that

$$
\nabla \bar{y}_{j}(x)+\frac{1}{\mathcal{V} \pi} \frac{x-\mathbf{x}_{j}}{\left|x-\mathbf{x}_{j}\right|^{2}}=\nabla \mathcal{G}_{\mathbf{x}_{j}}(x)
$$

we have proved Theorem 4.6.

Theorem 4.6 implies the following corollary.

Corollary 4.7. Given $\varepsilon>0$ and a convex lattice polygon $\mathrm{D} \subset \mathbb{R}^{2}$, for all $n$ sufficiently large,

$$
\sup _{e \in \mathrm{D}_{n, 1}^{*}}\left|\mathbf{d}^{*} G_{\mu^{*}}(e)\right|<\frac{1}{2} \text { for any } \mu \in \mathscr{M}_{n}^{\varepsilon}
$$

Proof. Let $e^{*} \in \mathrm{D}_{n, 1}^{*}$, and let $e_{i}^{*} \in \operatorname{argmin}\left\{\operatorname{dist}\left(e^{*}, e_{i}^{*}\right) \mid e_{i}^{*} \in \operatorname{supp}\{\mu\}\right\}$. Applying Theorem 4.6, and splitting $\mathcal{G}_{\mathrm{x}_{i}}(x)=\frac{1}{\mathcal{V} \pi} \log \left(\left|x-\mathrm{x}_{i}\right|\right)+\bar{y}_{i}(x)$, we obtain the estimate

$$
\left|\mathbf{d} G_{\mu^{*}}(e)\right| \leqq \sup _{e \in \mathrm{L}_{1}^{*}}\left|\mathbf{d}^{*} G^{\mathrm{L}^{*}}(e)\right|+n^{-1}\left[\frac{(m-1) \varepsilon}{\mathcal{V} \pi}+\sum_{i=1}^{m}\left\|\bar{y}_{i}\right\| \mathcal{X}\right]+O\left(n^{-1-\delta} \log (n)\right),
$$

where we recall the definition of the norm $\|\cdot\| \mathcal{X}$ from (4.8). Further, from Section 4.4 we have that $\left\|\bar{y}_{i}\right\|_{\mathcal{X}}$ is uniformly bounded for $x_{i} \in\{x \in \mathrm{D} \mid \operatorname{dist}(x, \partial \mathrm{D}) \geqq$ $\varepsilon\}$, and so applying statement (2) of Theorem 4.3, we have the result.

\subsection{Proof of Theorem 3.1}

We now complete the proof of Theorem 3.1 using the results above. Our first step is to verify the necessity of the equilibrium conditions given in (3.3).

Let $u$ be a locally stable equilibrium containing the dislocation configuration $\mu \in \mathscr{M}_{n}^{\varepsilon}$. By inspecting the proof of Lemma 5.1 in [2], it follows that if $\mathbf{d} u(e) \in$ $\frac{1}{2}+\mathbb{Z}$ for some $e \in \mathrm{D}_{n, 1}$, then there exist lower energy states arbitrarily close to $u$, 
and so any $\alpha \in[\mathbf{d} u]$ has $\|\alpha\|_{\infty}<\frac{1}{2}$. By definition, we have that $\mathbf{d} \alpha=\mu$. Finally, let $v \in \mathscr{W}\left(\mathrm{D}_{n, 0}\right)$; then for $t$ sufficiently small, $\|\alpha+t \mathbf{d} v\|_{\infty}<\frac{1}{2}$, hence

$$
E_{n}(u+t v ; u)=\int_{\mathrm{D}_{n, 1}} \psi(\alpha+t \mathbf{d} v)-\psi(\alpha)=\int_{\mathrm{D}_{n, 1}} \lambda t \alpha \mathbf{d} v+\frac{1}{2} \lambda t^{2}|\mathbf{d} v|^{2} .
$$

It follows that $(\alpha, \mathbf{d} v)=0$ for any $v \in \mathscr{W}\left(\mathrm{D}_{n, 0}\right)$, hence $\delta \alpha=0$.

Next, we show that if $\alpha$ satisfies the equilibrium conditions (3.3), then it is unique. Suppose that $\alpha$ and $\alpha^{\prime}$ satisfy (3.3). We define $\beta=\alpha-\alpha^{\prime}$, and note that $\beta^{*} \in \mathscr{W}_{0}\left(\mathrm{D}_{n, 1}^{*}\right)$ satisfies $\mathbf{d}^{*} \beta^{*}=0$ and $\delta^{*} \beta^{*}=0$. Since $n \mathrm{D}^{*}$ is simply connected, the former condition implies that $\beta^{*}=\mathbf{d}^{*} w$ for some $w \in \mathscr{W}_{0}\left(\mathrm{D}_{n, 0}^{*}\right)$, which must satisfy $\boldsymbol{\Delta}^{*} w=0$ : by the uniqueness of the solution proved in Lemma 4.1, it follows that $w=0$, hence $\beta=0$, and thus $\alpha=\alpha^{\prime}$.

Since $*$ is a bijection between $\mathscr{W}\left(D_{n, 1}\right)$ and $\mathscr{W}_{0}\left(D_{n, 1}^{*}\right)$, there exists $\alpha \in \mathscr{W}\left(D_{n, 1}\right)$ such that $\alpha^{*}=\mathbf{d}^{*} G_{\mu^{*}}$. Furthermore, by using (2.7), we have that

$$
\begin{gathered}
\mathbf{d} \alpha(e)=\boldsymbol{\delta}^{*} \mathbf{d}^{*} G_{\mu^{*}}\left(e^{*}\right)=\mu^{*}\left(e^{*}\right)=\mu(e), \text { for } e \in \mathrm{D}_{2}, \\
\text { and } \delta \alpha(e)=\left(\mathbf{d}^{*}\right)^{2} G_{\mu^{*}}\left(e^{*}\right)=0 \text { for } e \in \mathrm{D}_{n, 0} .
\end{gathered}
$$

Finally, we note that $\|\alpha\|_{\infty}=\left\|\mathbf{d} G_{\mu^{*}}\right\|_{\infty}$, hence applying Corollary 4.7 , it follows that $\alpha$ satisfies (3.3) if $n$ is sufficiently large.

To demonstrate that $\alpha \in\left[\mathbf{d} u_{\mu}\right]$ for some $u_{\mu} \in \mathscr{W}\left(\mathrm{D}_{n, 0}\right)$, fix $e^{\prime} \in \mathrm{D}_{n, 0}$, and define $u_{\mu}\left(e^{\prime}\right)=0$. Using the fact that $\mathrm{D}_{n}$ is path-connected, let $\gamma^{e}$ be the path such that $\partial \gamma^{e}=e^{\prime} \cup-e$, and define $u_{\mu}(e):=\int_{\gamma^{e}} \alpha$. Letting $b=\left[e_{0}, e_{1}\right] \in \mathrm{D}_{n, 1}$, we find that

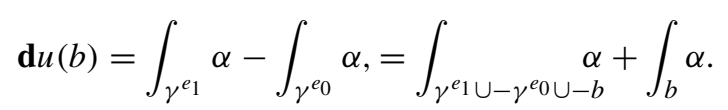

Noting that $\partial\left(\gamma^{e_{1}} \cup-\gamma^{e_{0}} \cup-b\right)=\emptyset$, we apply the fact that $\mathrm{D}_{n}$ is simply connected to assert that $\gamma^{e_{1}}-\gamma^{e_{0}}-b=\partial A$, for some $A \in \mathrm{D}_{2}$, hence

$$
\mathbf{d} u(b)=\alpha(b)+\int_{\partial A} \alpha=\alpha(b)+\int_{A} \mu \in \alpha(b)+\mathbb{Z} .
$$

It follows that $\alpha \in\left[\mathbf{d} u_{\mu}\right]$. To prove that $u_{\mu}$ is unique up to the equivalence (3.1), we note that if $\alpha \in[\mathbf{d} u]$ and $\alpha \in[\mathbf{d} v]$, then by the definition of a bond-length 1 -form (see Section 2.5), it follows that

$$
\mathbf{d} u(e)=\mathbf{d} v(e)+Z(e) \text { for all } e \in \mathrm{D}_{n, 1}, \quad \text { with } Z: \mathrm{D}_{n, 1} \rightarrow \mathbb{Z}
$$

Moreover, $\mathbf{d} Z=0$, so $Z=\mathbf{d} z$, and it is straightforward to check that $z: \mathrm{D}_{n, 0} \rightarrow$ $H+\mathbb{Z}$ for some $H \in \mathbb{R}$, completing the proof of Theorem 3.1. 


\section{Proof of Theorem 3.2}

This section is devoted to the proof of Theorem 3.2, and we proceed in several steps. We first demonstrate that there exists $u$ which 'solves' the min-max problem used to define $\mathcal{B}_{n}(\mu \rightarrow \nu)$ via a compactness method. We then identify necessary conditions for such a solution, and show that these necessary conditions identify a pair of bond-length 1-forms. The required bond-length 1-forms are then constructed via duality using an interpolation of dual Green's functions, and we verify that the necessary conditions are satisfied to conclude.

\subsection{The Min-Max Problem}

To establish existence of a solution, we transform the problem via taking the quotient of the space of deformations with respect to the equivalence relation defined in (3.1); in other words, we identify deformations 'up to lattice symmetries'. This space turns out to be compact, hence the existence of a critical point follows directly by a compactness argument.

5.1.1. Quotient Space Recall from (3.1) that $\sim$ is the equivalence relation on $u \in \mathscr{W}\left(\mathrm{D}_{n, 0}\right)$

$$
u \sim v \text { whenever } u=v+z+C \text { for some } z: \mathrm{D}_{n, 0} \rightarrow \mathbb{Z} \text { and some } C \in \mathbb{R} \text {. }
$$

Define the quotient space $\mathscr{Q}:=\mathscr{W}\left(\mathrm{D}_{n, 0}\right) / \sim$ of equivalence classes $\llbracket u \rrbracket$; we claim that this is a metric space when endowed with the metric

$$
d_{\mathscr{Q}}(\llbracket u \rrbracket, \llbracket v \rrbracket)=\|\alpha\|_{2}, \quad \text { where } \alpha \in[\mathbf{d} u-\mathbf{d} v], \quad \text { for any } u \in \llbracket u \rrbracket \text { and } v \in \llbracket v \rrbracket \text {. }
$$

If $u \sim v$, then $\mathbf{d} u \in \mathbf{d} v+\mathbb{Z}$, and hence [d $u]=[\mathbf{d} v]$. Symmetry is immediate, and $0 \in[\mathbf{d} u-\mathbf{d} v]$ implies that $u-v \sim 0$, hence $d_{\mathscr{Q}}(u, v)=0$ implies that $u \sim v$. Finally, for the triangle inequality, by checking cases it may be shown that

$$
\begin{aligned}
\beta \in & {[\mathbf{d} u], \beta^{\prime} \in[\mathbf{d} v] \text { and } \alpha \in[\mathbf{d} u+\mathbf{d} v] \text { imply that }|\alpha(e)| \leqq|\beta(e)| } \\
& +\left|\beta^{\prime}(e)\right| \text { for all } e \in \mathrm{D}_{n, 1} .
\end{aligned}
$$

The triangle inequality follows, and hence the metric is well-defined. Moreover, the space is complete and totally bounded, so the Heine-Borel theorem applies, and $\mathscr{Q}$ is compact. We recall that the mapping $u \mapsto \llbracket u \rrbracket$ is the natural embedding of $\mathscr{W}\left(\mathrm{D}_{n, 0}\right)$ in $\mathscr{Q}$.

5.1.2. Redefining the Energy As noted in Section 3.1, for any $u, u^{\prime}, v \in \mathscr{W}\left(\mathrm{D}_{n, 0}\right)$ such that $u \sim u^{\prime}, E_{n}(u, v)=E_{n}\left(u^{\prime}, v\right)$. It follows that the mapping $\widetilde{E}_{n}: \mathscr{Q} \rightarrow \mathbb{R}$,

$$
\widetilde{E}_{n}(\llbracket u \rrbracket):=E_{n}(u, v) \text { for some } u \in \llbracket u \rrbracket
$$

is well-defined. Suppose that $u \in \llbracket u \rrbracket$, and $u^{\prime} \in \llbracket u^{\prime} \rrbracket$, and that $\alpha \in\left[\mathbf{d} u-\mathbf{d} u^{\prime}\right]$. Then

$$
\begin{aligned}
\left|\widetilde{E}_{n}(\llbracket u \rrbracket)-\widetilde{E}_{n}\left(\llbracket u^{\prime} \rrbracket\right)\right| & =\left|\int_{\mathrm{D}_{n, 1}} \psi\left(\mathbf{d} u^{\prime}+\alpha\right)-\psi\left(\mathbf{d} u^{\prime}\right)\right| \lesssim C \int_{\mathrm{D}_{n, 1}}|\alpha| \\
& \lesssim\|\alpha\|_{2}=d_{\mathscr{Q}}\left(\llbracket u \rrbracket, \llbracket u^{\prime} \rrbracket\right),
\end{aligned}
$$

where we use the fact that $\psi$ is uniformly Lipschitz, and then apply the CauchySchwarz inequality. It follows that $\widetilde{E}_{n}$ is uniformly Lipschitz on $\mathscr{Q}$. 
5.1.3. Space of Continuous Paths Define the metric space $\mathrm{C}([0,1]$; $\mathscr{Q})$ of continuous functions from $[0,1]$ to $\mathscr{Q}$, with the usual metric

$$
d_{\mathscr{Q}}^{\infty}\left(\gamma, \gamma^{\prime}\right):=\sup _{t \in[0,1]} d_{\mathscr{Q}}\left(\gamma(t), \gamma^{\prime}(t)\right)
$$

The mapping $\gamma \mapsto \max _{t \in[0,1]} \widetilde{E}_{n}(\gamma(t))$ is continuous with respect to this metric, since $\widetilde{E}_{n}$ is uniformly continuous on $\mathscr{Q}$.

We suppose that $n$ is large enough such that the conclusion of Theorem 3.1 holds, and write $\llbracket u_{\mu} \rrbracket$ to mean the equivalence class containing $u_{\mu}$, which is the set of all locally stable equilibria corresponding to the dislocation positions $\mu \in \mathscr{M}_{n}^{\varepsilon}$. Define the sets of paths

$$
\begin{aligned}
\widetilde{\Gamma}_{n}(\mu \rightarrow v) & :=\left\{\gamma \in \mathrm{C}([0,1] ; \mathscr{Q}) \mid \gamma(0)=\llbracket u_{\mu} \rrbracket,\right. \\
\gamma(1) & \left.=\llbracket u^{v} \rrbracket, \alpha \in[\mathbf{d} \gamma(t)] \text { has } \mathbf{d} \alpha \in\{\mu, \nu\}, \forall t \in[0,1]\right\} ;
\end{aligned}
$$

this should be thought of as the set of paths through phase space which move dislocations from $\mu$ to $v$ without visiting any intermediate states.

5.1.4. Existence We recall that the energy barrier was defined to be

$$
\mathcal{B}_{n}(\mu \rightarrow v)=\inf _{\gamma \in \Gamma_{n}(\mu, v)} \sup _{t \in[0,1]} E_{n}\left(\gamma(t) ; u_{\mu}\right)
$$

The following lemma now demonstrates the existence of a transition state.

Lemma 5.1. If $n$ is sufficiently large, for any $\mu, v \in \mathscr{M}_{n}^{\varepsilon}$ such that $\Gamma_{n}(\mu \rightarrow \nu)$ is non-empty, there exists $u_{\uparrow} \in \mathscr{W}\left(\mathrm{D}_{n, 0}\right)$ such that

$$
E_{n}\left(u_{\uparrow} ; u_{\mu}\right)=\mathcal{B}_{n}(\mu \rightarrow v) .
$$

We will call $u_{\uparrow}$ a transition state for the transition from $\mu$ to $v$.

Proof. We first note that since $\mathscr{Q}$ is compact, $\mathrm{C}([0,1] ; \mathscr{Q})$ is compact. By assumption, $\Gamma_{n}(\mu \rightarrow v)$ is non-empty, and so the space $\widetilde{\Gamma}_{n}(\mu \rightarrow v)$ is non-empty by applying the natural embedding $\gamma(t) \mapsto \llbracket \gamma(t) \rrbracket$. Moreover, we have that

$$
\max _{t \in[0,1]} E_{n}\left(\gamma(t) ; u_{\mu}\right)=\max _{t \in[0,1]} \widetilde{E}_{n}(\llbracket \gamma(t) \rrbracket) .
$$

Since $\tilde{\gamma} \mapsto \max _{t \in[0,1]} \widetilde{E}_{n}(\tilde{\gamma}(t))$ is continuous, there exists a minimiser

$$
\tilde{\gamma} \in \operatorname{argmin}\left\{\max _{t \in[0,1]} \widetilde{E}_{n}(\tilde{\gamma}(t)) \mid \tilde{\gamma} \in \overline{\widetilde{\Gamma}_{n}(\mu \rightarrow \nu)}\right\},
$$

where $\widetilde{\Gamma}_{n}(\mu \rightarrow v)$ denotes the closure of $\widetilde{\Gamma}_{n}(\mu \rightarrow v)$ in $\mathscr{Q}$. As $t \mapsto \widetilde{E}_{n}(\tilde{\gamma}(t))$ is also continuous, it follows that there exists $u_{\uparrow} \in \llbracket u_{\uparrow} \rrbracket$ with $\llbracket u_{\uparrow} \rrbracket=\gamma\left(t^{*}\right) \in$ $\overline{\Gamma_{n}(\mu \rightarrow v)}$ for some $t^{*} \in[0,1]$, which satisfies

$$
E_{n}\left(u_{\uparrow} ; u_{\mu}\right)=\min _{\tilde{\gamma} \in \widetilde{\Gamma}_{n}(\mu \rightarrow v)} \max _{t \in[0,1]} \widetilde{E}_{n}(\tilde{\gamma}(t))=\min _{\gamma \in \Gamma_{n}(\mu \rightarrow v)} \max _{t \in[0,1]} E\left(\gamma(t) ; u_{\mu}\right) .
$$




\subsection{Necessary Conditions}

We now identify necessary conditions on the transition states identified in Lemma 5.1. We remark that the proof of the following lemma relies crucially on the particular choice of potential $\psi$.

Lemma 5.2. Suppose that $u_{\uparrow} \in \mathscr{W}\left(\mathrm{D}_{n, 0}\right)$ is a transition state for the transition from $\mu$ to $v$, where $v-\mu=b_{i}\left[\mathbb{1}_{q}-\mathbb{1}_{p}\right]$ and $q^{*}=p^{*}+$ a for some nearestneighbour direction in the dual lattice, a. Then $u_{\uparrow} \in\left\{u \in \mathscr{W}\left(\mathrm{D}_{n, 0}\right) \mid \alpha \in[\mathbf{d} u]\right.$ has $\left.\alpha(l)= \pm \frac{1}{2}\right\}$, where $l^{*}=\left[p^{*}, q^{*}\right]$, and moreover there exist exactly two $\alpha_{\uparrow}, \alpha_{\downarrow} \in\left[\mathbf{d} u_{\uparrow}\right]$, satisfying

(1) $\mathbf{d} \alpha_{\uparrow}=\mu, \mathbf{d} \alpha_{\downarrow}=v$,

(2) $\delta \alpha_{\uparrow}(a)=\delta \alpha_{\downarrow}(a)=0$ for all $a \pm \notin \partial l$,

(3) $\delta \alpha_{\uparrow}\left(e_{0}\right)+\delta \alpha_{\uparrow}\left(e_{1}\right)=0$ and $\delta \alpha_{\downarrow}\left(e_{0}\right)+\delta \alpha_{\downarrow}\left(e_{1}\right)=0$ for $e_{0}$ and $e_{1}$ such that $l=\left[e_{0}, e_{1}\right]$, and

(4) $-\alpha_{\uparrow}(l)=\alpha_{\downarrow}(l)=\frac{1}{2} b_{i}$.

Proof. We begin by proving that all transition states lie in the set

$$
B:=\left\{u \in \mathscr{W}\left(\mathrm{D}_{n, 0}\right) \mid \alpha \in[\mathbf{d} u] \text { has } \alpha(e)= \pm \frac{1}{2} \text { for some } e \in \mathrm{D}_{n, 1}\right\} .
$$

We remark that any $\gamma \in \Gamma_{n}(\mu \rightarrow \nu)$ must pass through $B$, since it is only on this set that we may have $\alpha, \alpha^{\prime} \in[\mathbf{d} \gamma(t)]$ with

$$
\mathbf{d} \alpha(p)=b_{i}, \quad \mathbf{d} \alpha(q)=0, \quad \text { and } \quad \mathbf{d} \alpha^{\prime}(p)=0, \quad \mathbf{d} \alpha^{\prime}(q)=b_{i} .
$$

Suppose that $\gamma \in \overline{\Gamma_{n}(\mu \rightarrow v)}$ solves the minimisation problem (3.4), and attains a transition state $u_{\uparrow}=\gamma\left(t^{*}\right)$ at $t=t^{*}$. Suppose further that $u_{\uparrow} \notin B$.

Taking an interval with $t^{*} \in\left[t_{1}, t_{2}\right]$ such that $\gamma(t) \notin B$ for all $t \in\left[t_{1}, t_{2}\right]$, and $\gamma\left(t_{1}\right), \gamma\left(t_{2}\right) \neq \gamma\left(t^{*}\right)$, we define

$$
\beta(t):= \begin{cases}\gamma(t) & t \notin\left[t_{1}, t_{2}\right], \\ \frac{t_{2}-t}{t_{2}-t_{1}} \gamma\left(t_{1}\right)+\frac{t-t_{1}}{t_{2}-t_{1}} \gamma\left(t_{2}\right) & t \in\left[t_{1}, t_{2}\right] .\end{cases}
$$

This is a valid competitor for the minimum problem, and moreover by using strict convexity of $\psi(x)$ for $x \in\left[n-\frac{1}{2}+, n+\frac{1}{2}\right]$ for any $n \in \mathbb{Z}$, we obtain

$$
\begin{aligned}
& E_{n}\left(\gamma\left(t^{*}\right) ; u_{\mu}\right) \leqq \sup _{t \in\left(t_{1}, t_{2}\right)} E_{n}\left(\beta(t) ; u_{\mu}\right)<\max \left\{E_{n}\left(\gamma\left(t_{1}\right) ; u_{\mu}\right), E_{n}\left(\gamma\left(t_{2}\right) ; u_{\mu}\right)\right\} \\
& \quad \leqq E_{n}\left(\gamma\left(t^{*}\right) ; u_{\mu}\right),
\end{aligned}
$$

which is a contradiction.

Suppose once more that $\gamma$ is a minimal path, and $\max _{t \in[0,1]} E\left(\gamma(t) ; u_{\mu}\right)$ attaining a transition state at $t=t^{*}$. Suppose also that $\alpha \in\left[\mathbf{d} \gamma\left(t^{*}\right)\right]$ has $\alpha(e)= \pm \frac{1}{2}$ for some $e \neq \pm l$. Let $a \in \partial e$ such that $e \notin \pm \partial l$. Then by considering $\gamma\left(t^{*}\right)+s \mathbb{1}_{a}$, and following the strategy of proof of Lemma 5.1 in [2], it may be checked that there exists $\delta>0$ such that for all $s \in[0, \delta)$ or for all $s \in(-\delta, 0]$,

(1) $\alpha \in\left[\mathbf{d}\left(\gamma\left(t^{*}\right)+s \mathbb{1}_{a}\right)\right]$ satisfies $\mathbf{d} \alpha \in\{\mu, v\}$, and 
(2) $E\left(\gamma\left(t^{*}\right)+s \mathbb{1}_{a} ; \gamma\left(t^{*}\right)\right)<0$ if $s \neq 0$.

By redefining $\gamma$ to pass through $\gamma\left(t^{*}\right)+s \mathbb{1}_{a}$ in a neighbourhood of $t^{*}$, it follows that $\gamma\left(t^{*}\right)$ cannot be a transition state, and hence if $u$ is a transition state with $\alpha \in[\mathbf{d} u]$, $\alpha(e)= \pm \frac{1}{2}$ if and only if $e= \pm l$. that

By considering paths $\beta$ which have $\beta\left(t^{*}\right)=\gamma\left(t^{*}\right)+s \mathbb{1}_{a}$ with $a \notin \partial l$, we obtain

$$
E_{n}\left(\gamma\left(t^{*}\right) ; u_{\mu}\right) \leqq E_{n}\left(\gamma\left(t^{*}\right)+s \mathbb{1}_{a} ; u_{\mu}\right)
$$

for all $s$ sufficiently small. If $\alpha \in\left[\mathbf{d} \gamma\left(t^{*}\right)\right]$, we have that

$$
\int_{D_{n, 1}} \alpha \mathbf{d} \mathbb{1}_{a}=\delta \alpha(a)=0 .
$$

By considering $\gamma\left(t^{*}\right)+s\left(\mathbb{1}_{e_{0}}+\mathbb{1}_{e_{1}}\right)$, where $l=\left[e_{0}, e_{1}\right]$, we obtain that

$$
\delta \alpha\left(e_{0}\right)+\delta \alpha\left(e_{1}\right)=0
$$

hence we have proved that a transition state must satisfy conditions (1)-(4).

Next, we prove that $\mathbf{d} \alpha_{\uparrow}=\mu, \alpha_{\uparrow}(l)=\frac{1}{2}$ and conditions (2) and (3) define a unique 1-form, which is an elastic strain at the transition state. Suppose that $\alpha_{\uparrow}$ and $\alpha_{\uparrow}^{\prime}$ satisfy these conditions. Defining $\theta:=\alpha_{\uparrow}-\alpha_{\uparrow}^{\prime}$, we have that $\mathbf{d} \theta=0$, hence $\theta=\mathbf{d} v$ for some 0 -form $v$ since $\mathrm{D}_{n}$ is simply connected. Furthermore, $\mathbf{d} v(l)=\theta(l)=0, \boldsymbol{\Delta} v(b)=s$ and $\boldsymbol{\Delta} v(c)=-s$ for some $s \in \mathbb{R}$. Then we have

$$
(\theta, \theta)=(\mathbf{d} v, \mathbf{d} v)=(\boldsymbol{\Delta} v, v)=s(v(b)-v(c))=s \mathbf{d} v(l)=0,
$$

implying that $\theta=0$, and hence $\alpha_{\uparrow}$ is unique. It may be similarly verified that $\alpha_{\downarrow}$ exists and is unique, completing the proof.

\subsection{Construction of the Transition State}

In Theorem 3.1, we found that the bond length 1-forms corresponding to local equilibria containing dislocations are related to dual Green's functions. By considering this relationship, it is natural to consider strains dual to interpolations of these Green's functions as possible candidates for the transition state $u$. We therefore define $G^{t}:=(1-t) G_{\mu^{*}}+t G_{v^{*}}$, where $t \in[0,1], G_{\mu^{*}}$ and $G_{v^{*}}$ solve (3.2). We note that for any $e^{*} \in \mathrm{D}_{n, 0}^{*}$,

$$
\begin{gathered}
\boldsymbol{\Delta}^{*} G^{t}\left(e^{*}\right)=(1-t) \boldsymbol{\Delta}^{*} G_{\mu^{*}}\left(e^{*}\right)+t \boldsymbol{\Delta}^{*} G_{v^{*}}\left(e^{*}\right)=(1-t) \mu^{*}\left(e^{*}\right)+t v^{*}\left(e^{*}\right) \\
\text { and in particular, } \boldsymbol{\Delta}^{*} G^{t}\left(p^{*}\right)=b_{j}(1-t) \text { and } \boldsymbol{\Delta}^{*} G^{t}\left(q^{*}\right)=b_{j} t
\end{gathered}
$$

As in Lemma 5.2, set $l \in \mathrm{D}_{n, 1}$ with $l^{*}=\left[p^{*}, q^{*}\right]$. Since Lemma 5.2 entails that the transition state must have $\alpha_{\downarrow}(l)=\frac{1}{2} b_{i}$, we choose $t \in[0,1]$ such that

$$
\begin{aligned}
\boldsymbol{\Delta}^{*} G^{t}\left(p^{*}\right)+\mathbf{d}^{*} G^{t}\left(l^{*}\right)=\frac{1}{2} b_{j}, & \Leftrightarrow \quad(1-t) b_{j}+\mathbf{d}^{*} G^{t}\left(l^{*}\right)=\frac{1}{2} b_{j}, \quad \text { and } \\
\boldsymbol{\Delta}^{*} G^{t}\left(q^{*}\right)-\mathbf{d}^{*} G^{t}\left(l^{*}\right)=\frac{1}{2} b_{j}, & \Leftrightarrow \quad t b_{j}-\mathbf{d}^{*} G^{t}\left(l^{*}\right)=\frac{1}{2} b_{j}
\end{aligned}
$$


Solving, we find that

$$
t=\frac{\frac{1}{2} b_{j}+\mathbf{d}^{*} G_{\mu^{*}}\left(l^{*}\right)}{b_{j}+\mathbf{d}^{*} G_{\mu^{*}}\left(l^{*}\right)-\mathbf{d}^{*} G_{\nu^{*}}\left(l^{*}\right)} .
$$

Noting that $\mathbf{d}^{*} G_{\mu^{*}}\left(l^{*}\right)=\mathbf{d}^{*} G_{\mathrm{L}^{*}}([0,0+\mathrm{a}])+o(1)=\frac{1}{\mathcal{K}^{*}} b_{j}+o(1)$ and similarly, $\mathbf{d}^{*} G_{v^{*}}\left(l^{*}\right)=-\frac{1}{\mathcal{K}^{*}} b_{j}+o(1)$, as $n \rightarrow \infty$ by applying Theorem 4.6 and statement (2) of Lemma 4.3, we see that $t \in[0,1]$; indeed, $t \rightarrow \frac{1}{2}$ as $n \rightarrow \infty$.

We now define $\alpha_{\uparrow}$ and $\alpha_{\downarrow}$ via

$$
\alpha_{\uparrow}^{*}\left(e^{*}\right):=\left\{\begin{array}{ll}
\mathbf{d}^{*} G^{t}\left(e^{*}\right) & e^{*} \neq \pm l^{*}, \\
\mp \frac{1}{2} b_{j} & e^{*}= \pm l^{*},
\end{array} \quad \text { and } \quad \alpha_{\downarrow}^{*}\left(e^{*}\right):= \begin{cases}\mathbf{d}^{*} G^{t}\left(e^{*}\right) & e^{*} \neq \pm l^{*}, \\
\mp \frac{1}{2} b_{j} & e^{*}= \pm l^{*}\end{cases}\right.
$$

where $t$ is given by (5.3). Letting $\alpha_{\mu} \in\left[\mathbf{d} u_{\mu}\right]$, for any $e \in \mathrm{D}_{2}$ with $e \neq p, q$, by duality we have

$$
\mathbf{d}\left[\alpha_{\uparrow}-\alpha_{\mu}\right](e)=\boldsymbol{\Delta}^{*} G^{t}\left(e^{*}\right)-\boldsymbol{\Delta}^{*} G_{\mu^{*}}\left(e^{*}\right)=t\left[\boldsymbol{\Delta}^{*} G_{\nu^{*}}\left(e^{*}\right)-\boldsymbol{\Delta}^{*} G_{\mu^{*}}\left(e^{*}\right)\right]=0 .
$$

Again, by duality we also have

$$
\begin{aligned}
& \mathbf{d}\left(\alpha_{\uparrow}-\alpha_{\mu}\right)(p)=\Delta^{*} G^{t}\left(p^{*}\right)+\mathbf{d}^{*} G^{t}\left(l^{*}\right)+\frac{1}{2} b_{j}-\Delta^{*} G_{\mu^{*}}\left(p^{*}\right)=0, \\
& \mathbf{d}\left(\alpha_{\uparrow}-\alpha_{\mu}\right)(q)=\Delta^{*} G^{t}\left(q^{*}\right)-\mathbf{d}^{*} G^{t}\left(l^{*}\right)-\frac{1}{2} b_{j}-\Delta^{*} G_{\mu^{*}}\left(p^{*}\right)=0 .
\end{aligned}
$$

Similarly, $\mathbf{d}\left(\alpha_{\downarrow}-\alpha_{v}\right)=0$. It follows therefore that there exist $v_{\uparrow}$ and $v_{\downarrow}$ such that $\alpha_{\uparrow} \in\left[\mathbf{d} u_{\mu}+\mathbf{d} v_{\uparrow}\right]$, and $\alpha_{\uparrow} \in\left[\mathbf{d} u_{\mu}+\mathbf{d} v_{\downarrow}\right]$.

We also note that if $a \notin \pm \partial l$ and $l=\left[e_{0}, e_{1}\right]$, then

$$
\begin{aligned}
& \boldsymbol{\delta}\left(\alpha_{\uparrow}-\alpha_{\mu}\right)(a)=\left(\mathbf{d}^{*}\right)^{2}\left(G^{t}-G_{\mu^{*}}\right)\left(a^{*}\right)=0, \\
& \boldsymbol{\delta}\left(\alpha_{\uparrow}-\alpha_{\mu}\right)\left(e_{0}\right)+\boldsymbol{\delta}\left(\alpha_{\uparrow}-\alpha_{\mu}\right)\left(e_{1}\right)=\left(\mathbf{d}^{*}\right)^{2}\left(G^{t}-G_{\mu^{*}}\right)\left(e_{0}^{*}\right)+\left(\mathbf{d}^{*}\right)^{2}\left(G^{t}-G_{\mu^{*}}\right)\left(e_{1}^{*}\right)=0 .
\end{aligned}
$$

It follows that $\alpha_{\uparrow}$ and $\alpha_{\downarrow}$ satisfy conditions (1)-(4) of Lemma 5.2, and hence we have constructed the bond-length one forms corresponding to the transition state.

Finally, we define $\gamma \in \Gamma_{n}(\mu \rightarrow v)$ via

$$
\gamma(t):= \begin{cases}u_{\mu}+2 t v_{\uparrow} & t \in\left[0, \frac{1}{2}\right] \\ u_{\mu}+v_{\uparrow}+(2 t-1) v_{\downarrow} & t \in\left(\frac{1}{2}, 1\right]\end{cases}
$$

which demonstrates that $\Gamma_{n}(\mu \rightarrow v)$ is non-empty, and hence $\mathcal{B}_{n}(\mu \rightarrow v)$ exists. 


\subsection{Proof of Theorem 3.2}

We now use the dual representation of $\alpha_{\uparrow}, \alpha_{\downarrow}, \alpha_{\mu}$ and $\alpha_{v}$ to give an asymptotic expression for $\mathcal{B}_{n}(\mu \rightarrow \nu)$ as $n \rightarrow \infty$. We use duality to compute

$$
\begin{aligned}
\mathcal{B}_{n}(\mu \rightarrow v)= & E_{n}\left(u_{\mu}+u_{\uparrow} ; u_{\mu}\right), \\
= & \frac{1}{2} \lambda\left[\left(\alpha_{\uparrow}, \alpha_{\uparrow}\right)-\left(\alpha_{\mu}, \alpha_{\mu}\right)\right], \\
= & \frac{1}{2} \lambda\left[\left(\mathbf{d}^{*} G^{t}, \mathbf{d}^{*} G^{t}\right)-\left(\mathbf{d}^{*} G_{\mu^{*}}, \mathbf{d}^{*} G_{\mu^{*}}\right)-\mathbf{d}^{*} G^{t}\left(l^{*}\right)^{2}+\frac{1}{4}\right], \\
= & \frac{1}{2} \lambda\left[2 t\left(\mathbf{d}^{*} G_{v^{*}}-\mathbf{d}^{*} G_{\mu^{*}}, \mathbf{d}^{*} G_{\mu^{*}}\right)\right. \\
& \left.+t^{2}\left(\mathbf{d}^{*} G_{\nu^{*}}-\mathbf{d}^{*} G_{\mu^{*}}, \mathbf{d}^{*} G_{v^{*}}-\mathbf{d}^{*} G_{\mu^{*}}\right)-\mathbf{d}^{*} G^{t}\left(l^{*}\right)^{2}+\frac{1}{4}\right], \\
= & \frac{1}{2} \lambda\left[2 t\left(\boldsymbol{\Delta}^{*} G_{v^{*}}-\boldsymbol{\Delta}^{*} G_{\mu^{*}}, G_{\mu^{*}}\right)\right. \\
& \left.+t^{2}\left(\boldsymbol{\Delta}^{*} G_{v^{*}}-\boldsymbol{\Delta}^{*} G_{\mu^{*}}, G_{v^{*}}-G_{\mu^{*}}\right)-\mathbf{d}^{*} G^{t}\left(l^{*}\right)^{2}+\frac{1}{4}\right], \\
= & \frac{1}{2} \lambda\left[t b_{j} \mathbf{d}^{*} G_{\mu^{*}}\left(l^{*}\right)+t b_{j} \mathbf{d}^{*} G^{t}\left(l^{*}\right)-\mathbf{d}^{*} G^{t}\left(l^{*}\right)^{2}+\frac{1}{4}\right] \\
= & \frac{1}{2} \lambda\left[t b_{j} \mathbf{d}^{*} G_{\mu^{*}}\left(l^{*}\right)+\frac{1}{2} b_{j} \mathbf{d}^{*} G^{t}\left(l^{*}\right)+\frac{1}{4}\right] \\
= & \frac{1}{2} \lambda\left[\frac{1}{2} b_{j} \mathbf{d}^{*} G_{\mu^{*}}\left(l^{*}\right)+\frac{1}{2} t b_{j}\left(\mathbf{d}^{*} G_{\mu^{*}}\left(l^{*}\right)+\mathbf{d}^{*} G_{v^{*}}\left(l^{*}\right)\right)+\frac{1}{4}\right],
\end{aligned}
$$

where we use (2.1) and the definition of $G_{\mu^{*}}$ and $G_{\nu^{*}}$ as Green's functions; to arrive at the penultimate line, we factorise and use (5.2), and use the definition of $G^{t}$ to obtain the final line. As a consequence of Theorem 4.6, we have

$$
\begin{aligned}
& b_{j} \mathbf{d}^{*} G_{\mu^{*}}\left(l^{*}\right)=b_{j}^{2} \mathbf{d} G^{\mathrm{L}^{*}}([0,0+\mathrm{a}])+n^{-1}\left[b_{j}^{2} \nabla \bar{y}_{j}\left(x_{j}\right) \cdot \mathrm{a}+\sum_{i \neq j} b_{j} b_{i} \nabla \mathcal{G}_{x_{i}}\left(x_{j}\right) \cdot \mathrm{a}\right]+o\left(n^{-1}\right), \\
& b_{j}\left[\mathbf{d}^{*} G_{\mu^{*}}\left(l^{*}\right)-\mathbf{d}^{*} G_{v^{*}}\left(l^{*}\right)\right]=2 b_{j}^{2} \mathbf{d} G^{\mathrm{L}}([0,0+\mathrm{a}])+o\left(n^{-1}\right), \\
& b_{j}\left[\mathbf{d}^{*} G_{\mu^{*}}\left(l^{*}\right)+\mathbf{d}^{*} G_{v^{*}}\left(l^{*}\right)\right]=2 n^{-1}\left[b_{j}^{2} \nabla \bar{y}_{j}\left(x_{j}\right) \cdot \mathrm{a}+\sum_{i \neq j} b_{j} b_{i} \nabla \mathcal{G}_{x_{i}}\left(x_{j}\right) \cdot \mathrm{a}\right]+o\left(n^{-1}\right)
\end{aligned}
$$

Using (5.6), it follows that

$$
\begin{aligned}
t & =\frac{\frac{1}{2} b_{j}+b_{j}^{2} \mathbf{d} G^{\mathrm{L}^{*}}([0,0+\mathrm{a}])+n^{-1}\left[b_{j}^{2} \nabla \bar{y}_{j}\left(x_{j}\right) \cdot \mathrm{a}+\sum_{i \neq j} b_{i} b_{j} \nabla \mathcal{G}_{x_{i}}\left(x_{j}\right) \cdot \mathrm{a}\right]+o\left(n^{-1}\right)}{b_{j}+2 b_{j}^{2} \mathbf{d} G^{\mathrm{L}^{*}}([0,0+\mathrm{a}])+o\left(n^{-1}\right)}, \\
& =\frac{1}{2}+n^{-1} \frac{b_{j} \nabla \bar{y}_{j}\left(x_{j}\right) \cdot \mathrm{a}+\sum_{i \neq j} b_{i} \nabla \mathcal{G}_{x_{i}}\left(x_{j}\right) \cdot \mathbf{a}}{1+2 b_{j} \mathbf{d} G^{\mathrm{L}^{*}}([0,0+\mathrm{a}])}+o\left(n^{-1}\right) .
\end{aligned}
$$

Substituting (5.5), (5.7) and (5.8) into (5.4), we obtain

$$
\begin{aligned}
\mathcal{B}_{n}(\mu \rightarrow \nu)= & \frac{1}{8} \lambda+\frac{1}{4} \lambda \mathbf{d} G^{\mathrm{L}^{*}}([0,0+\mathrm{a}]) \\
& +\frac{1}{2} \lambda n^{-1}\left[\nabla \bar{y}_{j}\left(x_{j}\right) \cdot \mathrm{a}+\sum_{i \neq j} b_{j} b_{i} \nabla \mathcal{G}_{x_{j}}\left(x_{i}\right) \cdot \mathrm{a}\right]+o\left(n^{-1}\right) .
\end{aligned}
$$

Finally, setting $c_{0}:=\frac{1}{8}+\frac{1}{4} \mathbf{d} G^{\mathrm{L}^{*}}([0,0+\mathrm{a}])$ completes the proof of Theorem 3.2. 


\section{Proofs of Large Deviations results}

This section is devoted to the proofs of the Large Deviations Principles. Section 6.1 verifies Theorem 3.3 by using the results of [21]. Theorem 3.4 is then split into the cases where $L^{*}$ is a Bravais lattice, i.e. $L=H x$ or $L=S q$, and where $L^{*}$ is a multi-lattice, i.e. where $L=\operatorname{Tr}$. These separate cases are covered by Lemma 6.1 and Lemma 6.2, and the proofs of these results constitute the remainder of the section.

\subsection{Proof of Theorem 3.3}

Where not otherwise stated, all references given in this section are to results in [21].

Conditions (1)-(4) assumed in Theorem 3.3 above are particular cases of assumptions of Theorem 6.14. The only additional conditions we need to verify to apply this theorem are first, that the equation

$$
F_{\delta}(x, f(x), \nabla f(x)):=f(x)-\delta \mathcal{H}(x, \nabla f(x))-h(x)=0,
$$

where $F_{\delta}: E \times \mathbb{R} \times \mathbb{R}^{N} \rightarrow \mathbb{R}$, satisfies a comparison principle for all $\delta>0$ sufficiently small, and second, that the domain of $H$ is dense in $\mathrm{C}(E ; \mathbb{R})$. The second condition is immediate, since $H$ is defined on $C^{1}(E ; \mathbb{R})$.

We recall that a comparison principle is the statement that viscosity sub- and supersolutions of (6.1) are globally ordered. When $x$ lies on the boundary of $M$, $\mathcal{H}$ vanishes, hence $F_{\delta}(x, r, p)=r-h(x)$ for all $x \in \partial M$. Thus any subsolution $\bar{f}$ and supersolution $\underline{f}$ must satisfy

$$
\bar{f}(x) \leqq \underline{f}(x) \text { for all } x \in \partial M .
$$

Theorem 3.3 in [15] asserts that $F_{\delta}$ satisfies a comparison principle on the interior of $M$ if

(1) There exists $\gamma>0$ such that

$$
\gamma(r-s) \leqq F_{\delta}(x, r, p)-F_{\delta}(x, s, p)
$$

for all $x$ in the interior of $M, r, s \in \mathbb{R}$ and $p \in \mathbb{R}^{N}$; and

(2) There exists a function $\omega:[0,+\infty) \rightarrow[0,+\infty)$ with $\lim _{t \rightarrow 0} \omega(t)=0$, such that

$$
F_{\delta}(x, r, \alpha(x-y))-F_{\delta}(y, r, \alpha(x-y)) \leqq \omega\left(\alpha|x-y|^{2}+|x-y|\right)
$$

for all $x$ and $y$ in the interior of $M, \gamma \in \mathbb{R}$ and $r \in \mathbb{R}$.

It is straightforward to verify that the former condition holds with $\gamma=1$ for $F_{\delta}$ as defined in (6.1); since we have assumed uniform continuity and differentiability of $\mathcal{H}$ on the interior of $M \times \mathbb{R}^{N}$, the second condition is also straightforward to verify, since $M \times\{\alpha(x-y) \mid x, y \in M\}$ is compact in $M \times \mathbb{R}^{N}$. Thus, a comparison principle holds on the entirety of $M$, and it follows that the conclusion of Theorem 6.14 holds, i.e. the sequence of Markov processes satisfies a Large Deviations Principle. 
To conclude that the rate function takes a variational form, we will first apply Corrolary 8.29. This requires us to check the conditions of Theorem 8.27. In the case considered here, the operators $\mathbf{H}_{\dagger}=\mathbf{H}_{\ddagger}=H$, thus we need only check that Conditions 8.9, 8.10 and 8.11 hold.

To verify Condition 8.9, we note the following, which demonstrate that each of the subconditions (1)-(5) are satisfied.

(1) In our case,

$$
A f(x, u)=u \cdot \nabla f(x),
$$

which is well-defined on $C^{1}(M ; \mathbb{R})$; this set separates points, so Condition 8.9.1 is verified.

(2) Here, $\Gamma:=M \times \mathbb{R}^{N}$ : for any $x_{0} \in M$, define $x(t)=x_{0}$ for all $t \in[0,+\infty)$ and $\lambda(d s \times d u)=\delta_{0}(d u) \times d s$ to verify Condition 8.9.2.

(3) Condition 8.9.3 is satisfied by assumption (5).

(4) Condition 8.9.4 is trivially satisfied by taking $\hat{K}=M$, since $M$ is compact.

(5) Condition 8.9.5 is satisfied due to our assumption that $\mathcal{L}$ satisfies the growth condition (3.9).

Condition 8.10 is satisfied upon taking $\lambda(d s \times d u)=\delta_{\dot{x}(s)}(u) d s \times d u$, where $x$ is the function whose existence was asserted in (3.10).

Finally, to verify Condition 8.11 , we follow the Legendre-Fenchel transform approach described in Section 8.3.6.2. Define

$$
q_{f}(x):= \begin{cases}\partial_{p} \mathcal{H}(x, \nabla f(x)) & x \in M \backslash \partial M, \\ 0 & x \in \partial M .\end{cases}
$$

This is well-defined, is continuous on the interior of $M$, and there exists a solution to the ODE $\dot{x}=q_{f}(x)$ with $x(0)=x_{0}$ and $x(t) \in M$ for all $t \in[0,+\infty)$, for any initial condition $x_{0} \in M$. Therefore, Condition 8.11 holds upon choosing $x$ to be this solution, and $\lambda(d u \times d s)=\delta_{q_{f}(x(s))}(d u) \times d s$.

We have thus verified the assumptions of Corollary 8.29, which allows us to conclude that the rate functional has a variational representation as a control problem, given in (8.18) in [21]. To conclude that the rate function takes the precise form we have here, where the solution to the minimisation problem over admissible controls is stated explicitly, we may apply an identical proof to that given for Theorem 10.22, noting that, under our assumptions, $I_{0}(x)$ is 0 if $x=x_{0}$, and $+\infty$ otherwise. We have therefore proved Theorem 3.3.

\subsection{Proof of Theorem 3.4: The Cases $\mathrm{L}=\mathrm{Hx}$ and $\mathrm{L}=\mathrm{Sq}$}

When the lattice is $\mathrm{Hx}$ or $\mathrm{Sq}$, the respective dual lattices are isomorphic to $\mathrm{Tr}$ and $\mathrm{Sq}$, and hence the set of nearest neighbour directions in the dual lattice is always the same; on the other hand, since $\mathrm{Tr}^{*}$ is isomorphic to $\mathrm{Hx}$, which is a multi-lattice, different techniques are required, and we therefore treat this case separately in the following section.

Take $f \in \mathrm{C}^{1}\left(\mathscr{M}_{\infty}^{\varepsilon} ; \mathbb{R}\right)$; as $\mathscr{M}_{\infty}^{\varepsilon}$ is compact, there exists a uniform modulus of continuity $\omega_{f}:[0,+\infty) \rightarrow[0,+\infty)$ with $\lim _{r \rightarrow 0} \omega_{f}(r)=0$, such that for 
$x=\left(x_{1}, \ldots, x_{m}\right) \in \mathscr{M}_{\infty}^{\varepsilon}$ and $y \in \mathscr{M}_{\infty}^{\varepsilon}$. Thus, for all $x \in \mathscr{M}_{\infty}^{\varepsilon}, n \in \mathbb{N}$ and $\mathrm{s} \in \mathbb{R}^{2}$ such that $x_{i}+\frac{1}{n} \mathrm{~s} \in \mathscr{M}_{\infty}^{\varepsilon}$, we have

$$
\left|f\left(x_{1}, \ldots, x_{j}+\frac{1}{n} \mathrm{~s}, \ldots, x_{m}\right)-f(x)-\frac{1}{n} \partial_{j} f(x) \cdot \mathrm{s}\right| \leqq \frac{|\mathrm{s}|}{n} \omega_{f}(|x-y|) .
$$

As $n \rightarrow \infty$ in the parameter regime we prescribed in Section 3.6, with $x$ in the interior of $\mathscr{M}_{\infty}^{\varepsilon}$, and a sequence $x_{n} \in \mathscr{M}_{n}^{\varepsilon}$ with $\operatorname{dist}\left(\iota_{n}\left(x_{n}\right), x\right) \rightarrow 0$ as $n \rightarrow \infty$, we have

$$
\begin{aligned}
H_{n} f \circ \iota_{n}\left(x_{n}\right)= & H f(x)+O\left(\omega_{f}\left(\operatorname{dist}\left(\iota_{n}\left(x_{n}\right), x\right)\right)\right), \text { setting } \\
H f(x):= & \sum_{i=1}^{m} \sum_{j=1}^{\mathcal{K}^{*}} A\left(\left[\exp \left(\partial_{j} f(x) \cdot \mathrm{s}_{j}\right)-1\right]\right. \\
& \left.\times \exp \left[-B\left(\nabla \bar{y}_{i}\left(x_{i}\right)+\sum_{k \neq j} b_{i} b_{k} \nabla \mathcal{G}_{x_{j}}\left(x_{i}\right)\right) \cdot \mathrm{s}_{j}\right]\right),
\end{aligned}
$$

where $\mathrm{s}_{j}$ are the nearest neighbour directions in $\mathrm{L}^{*}$. If $x_{n} \in \partial \mathscr{M}_{n}^{\varepsilon}$, then $H_{n} f \circ$ $\iota_{n}\left(x_{n}\right)=0$, so we define

$$
H f(x):=0 \text { for } x \in \partial \mathscr{M}_{\infty}^{\varepsilon} .
$$

Recall the definition of $\bar{y}_{i}$ as the solution to

$$
-\Delta \bar{y}_{i}=0 \text { in } \mathrm{D}, \quad \text { and } \quad \bar{y}_{i}(x)=\frac{1}{\mathcal{V}_{\pi}} \log \left(\left|x-x_{i}\right|\right) \text { on } \partial \mathrm{D} .
$$

Following the approach of [2] and [1], we define the renormalised energy for $x \in$ $\mathscr{M}_{\infty}^{\varepsilon}$ to be

$$
\mathcal{E}(x):=-\sum_{\substack{i, j=1 \\ i \neq j}}^{m} \frac{b_{i} b_{j}}{2 \mathcal{V} \pi} \log \left(\left|x_{i}-x_{j}\right|\right)+\sum_{i, j=1}^{m} b_{i} b_{j} \bar{y}_{i}\left(x_{j}\right)
$$

Recalling the definition of $\mathcal{G}_{y}$ from Theorem 3.2, we have that

$$
\partial_{i} \mathcal{E}(x)=\nabla \bar{y}_{i}\left(x_{i}\right)+\sum_{j \neq i} b_{i} b_{j} \nabla \mathcal{G}_{x_{j}}\left(x_{i}\right) .
$$

For $x$ in the interior of $\mathscr{M}_{\infty}^{\varepsilon}$, this allows us to write

$$
H f(x)=\sum_{i=1}^{m} \sum_{j=1}^{\mathcal{K}^{*}} A\left(\cosh \left[\left(\partial_{i} f(x)-B \partial_{i} \mathcal{E}(x)\right) \cdot \mathrm{a}_{j}\right]-\cosh \left[-B \partial_{i} \mathcal{E}(x) \cdot \mathrm{a}_{j}\right]\right)
$$

We define the Hamiltonian, $\mathcal{H}_{A, B}^{\mathrm{L}}: \mathscr{M}_{\infty}^{\varepsilon} \times \mathbb{R}^{2 m} \rightarrow \mathbb{R}$, as

$$
\begin{aligned}
& \mathcal{H}_{A, B}^{\mathrm{L}}(x, p) \\
& :=\left\{\begin{array}{c}
\sum_{i=1}^{m} \sum_{j=1}^{\mathcal{K}^{*}} A\left[\cosh \left(\left[p_{i}-B \partial_{i} \mathcal{E}(x)\right] \cdot \mathrm{s}_{j}\right)-\cosh \left(-B \partial_{i} \mathcal{E}(x) \cdot \mathrm{s}_{j}\right)\right] \\
x \in \mathscr{M}_{\infty}^{\varepsilon} \backslash \partial \mathscr{M}_{\infty}^{\varepsilon}, \\
0 x \in \partial \mathscr{M}_{\infty}^{\varepsilon},
\end{array}\right.
\end{aligned}
$$


where $p=\left(p_{1}, \ldots, p_{m}\right)$ with $p_{i} \in \mathbb{R}^{2}$ for each $i$. The Lagrangian is the LegendreFenchel transform (for further details on this topic, see Section 26 in [42]) of the Hamiltonian of $\mathcal{H}_{A, B}^{\mathrm{L}}$ with respect to its second argument, i.e.

$$
\mathcal{L}_{A, B}^{\mathrm{L}}(x, \xi):=\sup _{p \in \mathbb{R}^{2 m}}\left\{\langle\xi, p\rangle-\mathcal{H}_{A, B}^{\mathrm{L}}(x, p)\right\}
$$

where $\langle\cdot, \cdot\rangle$ is the inner product on $\mathbb{R}^{2 m}$ given by $\langle\xi, p\rangle:=\sum_{i=1}^{m} \xi_{i} \cdot p_{i}$. We now follow [10] in defining $\Psi_{A, B}^{\mathrm{Hx}}, \Psi_{A, B}^{\mathrm{Sq}}: \mathbb{R}^{2 m} \rightarrow \mathbb{R}$ via

$$
\begin{aligned}
& \Psi_{A, B}^{\mathrm{Hx}}(f):=\sum_{i=1}^{m} \sum_{j=1}^{6} \frac{A}{B}\left[\cosh \left(B f_{i} \cdot \mathrm{a}_{j}\right)-1\right], \\
& \Psi_{A, B}^{\mathrm{Sq}}(f):=\sum_{i=1}^{m} \sum_{j=1}^{4} \frac{A}{B}\left[\cosh \left(B f_{i} \cdot \mathrm{e}_{j}\right)-1\right],
\end{aligned}
$$

which permits us to write

$$
\mathcal{H}_{A, B}^{\mathrm{L}}(x, p)=B\left[\Psi_{A, B}^{\mathrm{L}}\left(\frac{1}{B} p-\nabla \mathcal{E}(x)\right)-\Psi_{A, B}^{\mathrm{L}}(-\nabla \mathcal{E}(x))\right] .
$$

$\Psi_{A, B}^{\mathrm{L}}$ is (strictly) convex, and hence has a convex dual, given by its LegendreFenchel transform, denoted $\Phi_{A, B}^{\mathrm{L}}$. Moreover, by properties of the Legendre-Fenchel transform, we have that

$$
\begin{aligned}
\nabla \Phi_{A, B}^{\mathrm{L}}(\xi)=\frac{1}{B} p-\nabla \mathcal{E}(x) & \Leftrightarrow \xi=\nabla \Psi_{A, B}^{\mathrm{L}}\left(\frac{1}{B} p-\nabla \mathcal{E}(x)\right) \\
& \Leftrightarrow p \in \underset{p^{\prime}}{\operatorname{argmax}}\left\{\left\langle\xi, p^{\prime}\right\rangle-\mathcal{H}_{A, B}^{\mathrm{L}}\left(x, p^{\prime}\right)\right\} .
\end{aligned}
$$

Using this fact, we have that

$$
\begin{aligned}
\mathcal{L}_{A, B}^{\mathrm{L}}(x, \xi)= & \left\langle B \nabla \Phi_{A, B}^{\mathrm{L}}(\xi)+B \nabla \mathcal{E}(x), \xi\right\rangle-\mathcal{H}_{A, B}^{\mathrm{L}}\left(x, B \nabla \Phi_{A, B}^{\mathrm{L}}(\xi)+B \nabla \mathcal{E}(x)\right) \\
= & \left\langle B \nabla \Phi_{A, B}^{\mathrm{L}}(\xi)+B \nabla \mathcal{E}(x), \xi\right\rangle-B \Psi_{A, B}^{\mathrm{L}}\left(\nabla \Phi_{A, B}^{\mathrm{L}}(\xi)\right) \\
& +B \Psi_{A, B}^{\mathrm{L}}(-\nabla \mathcal{E}(x))
\end{aligned}
$$

Using the property that $\langle u, v\rangle=\Psi_{A, B}^{\mathrm{L}}(u)+\Phi_{A, B}^{\mathrm{L}}(v)$, we then have

$$
\mathcal{L}_{A, B}^{\mathrm{L}}(x, \xi)=B \Phi_{A, B}^{\mathrm{L}}(\xi)+B \Psi_{A, B}^{\mathrm{L}}(-\nabla \mathcal{E}(x))+B\langle\nabla \mathcal{E}(x), \xi\rangle,
$$

which leads us to define the rate functional $\mathcal{J}_{A, B}^{\mathrm{L}}: \mathrm{D}\left([0, T] ; \mathscr{M}_{\infty}^{\varepsilon}\right) \rightarrow \mathbb{R}$ with

$$
\begin{aligned}
& \mathcal{J}_{A, B}^{\mathrm{L}}(x):= \begin{cases}\int_{0}^{\infty} \mathcal{L}_{A, B}^{\mathrm{L}}(x, \dot{x}) \mathrm{d} t & x \in \mathrm{W}^{1,1}\left([0,+\infty) ; \mathscr{M}_{\infty}^{\varepsilon}\right), \quad \text { and } \\
+\infty & \text { otherwise, }\end{cases} \\
& \mathcal{L}_{A, B}^{\mathrm{L}}(x, q) \\
& := \begin{cases}\Phi_{A, B}^{\mathrm{L}}(q)+\Psi_{A, B}^{\mathrm{L}}(-\nabla \mathcal{E}(x))+\langle\nabla \mathcal{E}(x), q\rangle & x \in \mathscr{M}_{\infty}^{\varepsilon} \backslash \partial \mathscr{M}_{\infty}^{\varepsilon}, \\
0 & x \in \partial \mathscr{M}_{\infty}^{\varepsilon} \text { and } q=0, \\
+\infty & x \in \partial \mathscr{M}_{\infty}^{\varepsilon} \text { and } q \neq 0 .\end{cases}
\end{aligned}
$$


We may now state the following result, asserting a Large Deviation Principle for the sequence of processes in this case.

Lemma 6.1. Suppose that $\mathrm{L}=\mathrm{Hx}$ or $\mathrm{L}=\mathrm{Sq}$, and that $X_{0}^{n}=\iota_{n}\left(x^{n}\right)$ where $x^{n} \rightarrow x_{0} \in \mathscr{M}_{n}^{\varepsilon}$ as $n \rightarrow \infty$ with $\delta>\varepsilon$. Then the processes $X_{t}^{n}$ satisfy a Large Deviations principle with good rate function $\mathcal{J}_{A, B}^{\mathrm{L}}$.

This result is very similar to those obtained in Chapter 5, Section 2 of [22], or Section 10.3 of [21], the main difference being that there is no diffusive part of the process. We also refer the reader to [11] for related results concerning a discrete-time model on a lattice.

Proof. As stated, we wish to apply Theorem 3.3 to prove Lemma 6.1. The main conditions we are required to check are (3), (4) and (5), since conditions (1) and (2) are straightforward to check when $M_{n}=\mathscr{M}_{n}^{\varepsilon}, M=\mathscr{M}_{\infty}^{\varepsilon}$, and $\iota_{n}$ is as defined in (3.6).

Verifying Condition (3) $\quad \mathscr{M}_{n}^{\varepsilon}$ is a finite state space and is endowed with a topology which is equivalent to the discrete topology. Therefore, by the Lebesgue Decomposition Theorem (see for example Theorem 3 of Section 1.6 in [19]) all measures $\mu$ on $\mathscr{M}_{n}^{\varepsilon}$ may be expressed as

$$
\mu(d x)=\sum_{y \in \mathscr{M}_{n}^{\varepsilon}} f(y) \delta_{y}(d x),
$$

where the density $f: \mathscr{M}_{n}^{\varepsilon} \rightarrow \mathbb{R}$ is continuous (as are all real-valued functions on $\left.\mathscr{M}_{n}^{\varepsilon}\right)$. Next, fix a probability measure $\mu_{y}(d x, 0)=\delta_{y}(d x)$ on $\mathscr{M}_{n}^{\varepsilon}$, and define

$$
\mu_{y}(d x, t)=\sum_{z \in \mathscr{M}_{n}^{\varepsilon}} f_{y}(z, t) \delta_{z}(d x),
$$

where $f_{y}: \mathscr{M}_{n}^{\varepsilon} \times[0,+\infty) \rightarrow \mathbb{R}$ solves $\partial_{t} f_{y}(x, t)=\Omega_{n}^{T} f(x, t)$ with $\Omega_{n}^{T}$ being the adjoint of $\Omega_{n}$, and $f_{y}(x, 0)=\delta_{y}(x)$. It is straightforward to see that $f_{y}$ exists and is unique, since $\Omega_{n}^{T}$ is a bounded linear operator, and therefore the ODE system $\partial_{t} f_{y}=\Omega_{n}^{T} f_{y}$ has a unique solution for all time, so the martingale problem is well-posed. Moreover, the mapping from $y$ to $\mu_{y}$ is trivially measurable, since the topology on $M_{n}$ is the discrete topology.

Verifying Condition (4) $\mathcal{H}_{A, B}^{\mathrm{L}}$ clearly satisfies the regularity conditions required, since $\mathcal{E}$ is harmonic on the interior of $M$, and cosh is smooth, and hence $\mathcal{H}_{A, B}^{\mathrm{L}}$ is smooth on the interior of $M \times \mathbb{R}^{2 m}$. The third condition holds by definition, and since $x \mapsto \cosh (B x \cdot \mathrm{a})$ is a convex function on $\mathbb{R}^{2}$ for any fixed $\mathrm{a} \in \mathbb{R}^{2}, \mathcal{H}_{A, B}^{\mathrm{L}}$ is convex in $p$ for any $x \in \mathscr{M}_{\infty}^{\varepsilon}$.

We take $g=H f$ for $f \in \mathrm{C}^{1}\left(\mathscr{M}_{\infty}^{\varepsilon} ; \mathbb{R}\right)$ and $f_{n}=f \circ \iota_{n}$, which trivially satisfies the required convergence condition. Since $f \in \mathrm{C}^{1}\left(\mathscr{M}_{\infty}^{\varepsilon} ; \mathbb{R}\right)$, it is straightforward to check that $g_{n}=H_{n} f_{n}$ is uniformly bounded, since $\nabla f$ is uniformly continuous, and $\mathcal{H}_{A, B}^{\mathrm{L}}$ is smooth, so the convergence statement made at the beginning of Section 6.2 holds uniformly for sequences $\iota_{n}\left(x_{n}\right)$ which approximate points in the interior 
of $\mathscr{M}_{\infty}^{\varepsilon}$. When $(x, p)$ is in the interior of $\mathscr{M}_{\infty}^{\varepsilon} \times \mathbb{R}^{2 m}, \mathcal{H}_{A, B}^{\mathrm{L}}$ is continuous, so verification of (3.8) follows from the same arguments. When $x \in \partial \mathscr{M}_{\infty}^{\varepsilon}$, there are two possible limits, either 0 or the limiting value for $\lim _{y \rightarrow x} \mathcal{H}_{A, B}^{\mathrm{L}}(y, p)$ for sequences of points $y$ lying in the interior of $\mathscr{M}_{\infty}^{\varepsilon}$. Supposing $\iota_{n}\left(x_{n}\right) \rightarrow x \in \partial M$, we therefore have

$$
\begin{aligned}
\min \left\{\lim _{y \rightarrow x} \mathcal{H}_{A, B}^{\mathrm{L}}(y, p), 0\right\} & \leqq \liminf _{n \rightarrow \infty} H_{n} f_{n}\left(x_{n}\right) \leqq \limsup _{n \rightarrow \infty} H_{n} f_{n}\left(x_{n}\right) \\
& \leqq \max \left\{\lim _{y \rightarrow x} \mathcal{H}_{A, B}^{\mathrm{L}}(y, p), 0\right\},
\end{aligned}
$$

which verifies the statement (3.8).

Verifying Condition (5) For $x \in \partial \mathscr{M}_{\infty}^{\varepsilon}$, we have that

$$
\mathcal{L}_{A, B}^{\mathrm{L}}(x, \xi)=\left\{\begin{array}{ll}
0 & \xi=0, \\
+\infty & \xi \neq 0
\end{array},\right.
$$

so (3.9) is trivially satisfied. Next, using hyperbolic trigonometric identities and the fact that $|\sinh (x)| \leqq \cosh (x)$ for all $x \in \mathbb{R}$, we obtain

$$
\cosh ([p-\nabla \mathcal{E}(x)] \cdot \mathrm{a})-\cosh ([-\nabla \mathcal{E}(x)] \cdot \mathrm{a}) \leqq 2 \cosh (|p|) \cosh (|\nabla \mathcal{E}(x)|) .
$$

Applying this estimate to the definition of $\mathcal{H}_{A, B}^{\mathrm{L}}$, we find that for some $M$ sufficiently large,

$$
\mathcal{H}_{A, B}^{\mathrm{L}}(x, p) \leqq M \sum_{i=1}^{m} \cosh \left(\left|p_{i}\right|\right) .
$$

Define $\psi(y):=M \cosh (|y|)$ for $y \in \mathbb{R}^{2}$; it may be verified that the LegendreFenchel transform of this function, $\psi^{*}$, is

$$
\psi^{*}(y)=|y| \sinh ^{-1}\left(\frac{|y|}{M}\right)-\sqrt{1+\frac{|y|^{2}}{M^{2}}} .
$$

By the ordering properties of the Legendre-Fenchel transform, we therefore have

$$
\mathcal{L}_{A, B}^{\mathrm{L}}(x, \xi) \geqq \sum_{i=1}^{m} \psi^{*}\left(\xi_{i}\right),
$$

and since $\sinh ^{-1}(r) \rightarrow \infty$ as $r \rightarrow \infty$, we have that $\frac{\psi\left(\xi_{i}\right)}{\left|\xi_{i}\right|} \rightarrow \infty$ as $\left|\xi_{i}\right| \rightarrow \infty$, thus $\mathcal{L}_{A, B}^{\mathrm{L}}$ satisfies (3.9).

Next, recalling that $\Psi_{A, B}^{\mathrm{L}}$ and $\Phi_{A, B}^{\mathrm{L}}$ are conjugate functions, we have that for any $\alpha, \beta \in \mathbb{R}^{2 m}$,

$$
\Psi_{A, B}^{\mathrm{L}}(\alpha)+\Phi_{A, B}^{\mathrm{L}}(\beta) \geqq\langle\alpha, \beta\rangle,
$$

where equality holds if and only if $\beta=\nabla \Psi_{A, B}^{\mathrm{L}}(\alpha)$. This implies that $\mathcal{L}_{A, B}^{\mathrm{L}}(x, \dot{x}) \geqq$ 0 for all $(x, \dot{x}) \in \mathscr{M}_{\infty}^{\varepsilon} \times \mathbb{R}^{2 m}$, and $\mathcal{L}_{A, B}^{\mathrm{L}}(x, \dot{x})=0$ when $x$ lies in the interior of $\mathscr{M}_{\infty}^{\varepsilon}$ if and only if

$$
\dot{x}=\nabla \Psi_{A, B}^{\mathrm{L}}(-\nabla \mathcal{E}(x))
$$


Given that the function on the right-hand side is uniformly Lipschitz for $x$ in the interior of $\mathscr{M}_{\infty}^{\varepsilon}$, it follows that there exists a solution $x \in \mathrm{C}([0, T] ; M)$ to the ODE

$$
\dot{x}(t)=\nabla \Psi_{A, B}^{\mathrm{L}}(-\nabla \mathcal{E}(x(t))), \quad x(0)=x_{0},
$$

where $T$ is chosen such that $x(T) \in \partial \mathscr{M}_{\infty}^{\varepsilon}$, and $x_{0}$ lies in the interior of $\mathscr{M}_{\infty}^{\varepsilon}$. Then, setting $x(t)=x(T)$ for all $t>T$, we have that $\dot{x}(t)=0$ for all $t>T$, and thus

$$
\int_{0}^{\infty} \mathcal{L}_{A, B}^{\mathrm{L}}(x(t), \dot{x}(t)) \mathrm{d} t=0
$$

we have therefore verified condition (5) of Theorem 3.3, so applying its conclusion, we have proved the result.

To conclude the proof of Theorem 3.4, we note that, by properties of the Legendre-Fenchel transform, for $x \in \mathscr{M}_{\infty}^{\varepsilon} \backslash \partial \mathscr{M}_{\infty}^{\varepsilon}, \mathcal{L}_{A, B}^{\mathrm{L}}(x, q) \geqq 0$,

$$
\mathcal{L}_{A, B}^{\mathrm{L}}(x, q)=0 \text { if and only if } q=\nabla \Psi_{A, B}^{\mathrm{L}}(-\nabla \mathcal{E}(x)),
$$

and by definition, $\mathcal{M}_{A, B}^{\mathrm{L}}=\nabla \Psi_{A, B}^{\mathrm{L}}$.

\subsection{Proof of Theorem 3.4: The Case $\mathrm{L}=\mathrm{Tr}$}

The case where $L=\operatorname{Tr}$ is more complicated than the cases treated above, since $\mathrm{Tr}^{*}$ is isomorphic to $\mathrm{Hx}$, which is a multi-lattice rather than a simple Bravais lattice. The value of $\Omega_{n} f$ therefore oscillates depending upon the specific sublattice on which each dislocation lies, and so the verification of the convergence condition in Theorem 3.3 requires an additional step in this case. The technique which we use to prove convergence bears significant similarities to the use of a periodic 'corrector' as used in the theory of homogenisation for differential operators with rapidly oscillating coefficients, and our approach may be viewed as the discrete analogue of the strategy used in Example 1.10 in [21].

For clarity, we first fix some notation which we use throughout the proof: recall from Section 2.3 the definition of $\mathrm{a}_{i}$, and the fact that $\operatorname{Tr}^{*}$ is the union of 2 translated copies of $\frac{\sqrt{3}}{3} \operatorname{Tr}$. It will therefore be convenient to define

$$
\mathrm{a}_{i}^{*}=\frac{1}{3}\left(\mathrm{a}_{2 i}+\mathrm{a}_{2 i-1}\right) \text { for } i=1,2,3, \quad \operatorname{Tr}_{+}^{*}:=\operatorname{Tr}+\mathrm{a}_{1}^{*}, \quad \text { and } \operatorname{Tr}_{-}^{*}:=\operatorname{Tr}-\mathrm{a}_{2}^{*}
$$

By definition, we have that $\operatorname{Tr}^{*}=\operatorname{Tr}_{+}^{*} \cup \operatorname{Tr}_{-}^{*}$; the subscripts refer to the fact that the nearest neighbour directions in $\mathrm{Tr}^{*}$ are

$$
\left\{\mathrm{a}_{1}^{*}, \mathrm{a}_{2}^{*}, \mathrm{a}_{3}^{*}\right\} \text { for } e^{*} \in \operatorname{Tr}_{+}^{*} \text { and }\left\{-\mathrm{a}_{1}^{*},-\mathrm{a}_{2}^{*},-\mathrm{a}_{3}^{*}\right\} \text { for } e^{*} \in \operatorname{Tr}_{-}^{*} \text {. }
$$


With this notation, if $\mu^{*}=\left(e_{1}^{*}, \ldots, e_{m}^{*}\right)$ with $r_{\infty}\left(\mu^{*}, x\right)=O\left(n^{-1}\right)$, we have

$$
\begin{aligned}
\Omega_{n} f(\mu)= & \sum_{i \mid e_{i}^{*} \in \operatorname{Tr}_{+}^{*}} \sum_{j=1}^{3}\left(n A \exp \left[-B \partial_{i} \mathcal{E}(x) \cdot \mathrm{a}_{j}^{*}+o(1)\right]\right. \\
& \left.\times\left[f\left(e_{1}^{*}, \ldots, e_{i}^{*}+\mathrm{a}_{j}^{*}, \ldots, e_{m}^{*}\right)-f(\mu)\right]\right) \\
& +\sum_{i \mid e_{i}^{*} \in \operatorname{Tr}_{-}^{*}} \sum_{j=1}^{3}\left(n A \exp \left[B \partial_{i} \mathcal{E}(x) \cdot \mathrm{a}_{j}^{*}+o(1)\right]\right. \\
& \left.\times\left[f\left(e_{1}^{*}, \ldots, e_{i}^{*}-\mathrm{a}_{j}^{*}, \ldots, e_{m}^{*}\right)-f(\mu)\right]\right) .
\end{aligned}
$$

We see that the generator oscillates in value depending upon whether each $e_{i}^{*} \in \mathrm{Tr}_{+}^{*}$ or $e_{i}^{*} \in \mathrm{Tr}_{-}^{*}$. To obtain a Large Deviations Principle, we must show that the nonlinear generator converges in the sense of condition (4) in Theorem 3.3. We suppose that $f \in \mathrm{C}^{1}\left(\mathscr{M}_{\infty}^{\varepsilon} ; \mathbb{R}\right)$, and define a sequence $f_{n}(\mu)=f \circ \iota_{n}(\mu)+\frac{1}{n} h_{f}\left(\iota_{n}(\mu) ; \mu\right)$, where $h_{f}: \mathscr{M}_{\infty}^{\varepsilon} \times\left(\operatorname{Tr}^{*}\right)^{m} \rightarrow \mathbb{R}$ will be defined shortly. For convenience, we also define $T_{\mathrm{s}}^{i} \mu:=\left(e_{1}, \ldots, e_{i}+\mathrm{s}, \ldots, e_{m}\right)$, and calculate

$$
\begin{aligned}
H_{n} f(\mu)= & \frac{1}{n} \mathrm{e}^{-n f(\mu)} \Omega_{n} \mathrm{e}^{n f}(\mu) \\
= & A \sum_{i \mid e_{i}^{*} \in \operatorname{Tr}_{+}^{*}} \sum_{j=1}^{3}\left(\exp \left[-B \partial_{i} \mathcal{E}(x) \cdot \mathrm{a}_{j}^{*}\right]\right. \\
& \left.\times\left[\exp \left(\partial_{i} f(x) \cdot \mathrm{a}_{j}^{*}+h_{f}\left(x, e_{1}, T_{\mathrm{a}_{j}^{*}}^{i} \mu\right)-h_{f}(x, \mu)\right)-1\right]\right) \\
& +A \sum_{i \mid e_{i}^{*} \in \operatorname{Tr}_{-}^{*}} \sum_{j=1}^{3}\left(\exp \left[B \partial_{i} \mathcal{E}(x) \cdot \mathrm{a}_{j}^{*}\right]\right. \\
& \left.\times\left[\exp \left(-\partial_{i} f(x) \cdot \mathrm{a}_{j}^{*}+h_{f}\left(x, T_{-\mathrm{a}_{j}^{*}}^{i} \mu\right)-h_{f}(x, \mu)\right)-1\right]\right) \\
& +o\left(n^{-1}\right) .
\end{aligned}
$$

Our aim is now to define $h_{f}$ such that for some $g \in \mathrm{C}\left(\mathscr{M}_{\infty}^{\varepsilon} ; \mathbb{R}\right)$,

$$
\sup _{\mu \in \mathscr{M}_{n}^{\varepsilon}}\left|H_{n}\left(f \circ \iota_{n}+\frac{1}{n} h_{f}\right)(\mu)-g \circ \iota_{n}(\mu)\right| \rightarrow 0 \text { as } n \rightarrow \infty \text {. }
$$

As long as $h_{f}(x, \mu)$ is uniformly bounded for $(x, \mu) \in \mathscr{M}_{\infty}^{\varepsilon} \times\left(\operatorname{Tr}^{*}\right)^{m}$, this will imply the convergence condition required in Theorem 3.3. We make the ansatz that $h_{f}\left(x ; e_{1}^{*}, \ldots, e_{m}^{*}\right)=\sum_{i=1}^{m} h_{f, i}\left(x ; e_{i}^{*}\right), \quad$ where $h_{f, i}\left(x ; e_{i}^{*}\right)=\left\{\begin{array}{l}h_{f, i}^{+}(x) e_{i}^{*} \in \mathrm{Tr}_{+}^{*}, \\ h_{f, i}^{-}(x) e_{i}^{*} \in \operatorname{Tr}_{-}^{*}\end{array}\right.$ thus, each $h_{f, i}: \mathscr{M}_{\infty}^{\varepsilon} \times \operatorname{Tr}^{*} \rightarrow \mathbb{R}$ depends only on whether $e_{i}^{*} \in \operatorname{Tr}_{+}^{*}$ or $e_{i}^{*} \in \operatorname{Tr}_{-}^{*}$. In order that $H_{n}\left(f \circ \iota_{n}+\frac{1}{n} h_{f}\right)-g \circ \iota_{n}$ tends to zero independently of the choice 
of sublattice for each $e_{i}^{*}$, we then choose $h_{f, i}^{ \pm}(x)$ to satisfy the 'corrector problem'

$$
\begin{aligned}
g(x)= & \sum_{i=1}^{m} \sum_{j=1}^{3} A\left[\exp \left(\left[\partial_{i} f(x)-B \partial_{i} \mathcal{E}(x)\right] \cdot \mathrm{a}_{j}^{*}+h_{f, i}^{-}(x)-h_{f, i}^{+}(x)\right)\right. \\
& \left.-\exp \left(-B \partial_{i} \mathcal{E}(x) \cdot \mathrm{a}_{j}^{*}\right)\right] \\
= & \sum_{i=1}^{m} \sum_{j=1}^{3} A\left[\exp \left(\left[B \partial_{i} \mathcal{E}(x)-\partial_{i} f(x)\right] \cdot \mathrm{a}_{j}^{*}+h_{f, i}^{+}(x)-h_{f, i}^{-}(x)\right)\right. \\
& \left.-\exp \left(B \partial_{i} \mathcal{E}(x) \cdot \mathrm{a}_{j}^{*}\right)\right] .
\end{aligned}
$$

Equating terms which contain $\pm\left[h_{f, i}^{+}(x)-h_{f, i}^{-}(x)\right]$ and solving, we set

$$
h_{f, i}^{ \pm}(x)= \pm \frac{1}{2} \log \left(\frac{\left(\gamma_{i}^{+}-\gamma_{i}^{-}\right)+\sqrt{\left(\gamma_{i}^{+}-\gamma_{i}^{-}\right)^{2}+4 \delta_{i}^{+} \delta_{i}^{-}}}{2 \delta_{i}^{+}}\right),
$$

and thus $g(x)=A \sum_{i=1}^{m} \sqrt{\frac{1}{4}\left(\gamma_{i}^{+}+\gamma_{i}^{-}\right)^{2}+\delta_{i}^{+} \delta_{i}^{-}-\gamma_{i}^{+} \gamma_{i}^{-}}-\frac{1}{2}\left(\gamma_{i}^{+}+\gamma_{i}^{-}\right)$,

$$
\begin{aligned}
\text { where } \gamma_{i}^{ \pm} & =\sum_{j=1}^{3} \exp \left(\mp B \partial_{i} \mathcal{E}(x) \cdot \mathrm{a}_{j}^{*}\right) \\
\text { and } \delta_{i}^{ \pm} & =\sum_{j=1}^{3} \exp \left( \pm\left[\partial_{i} f(x)-B \partial_{i} \mathcal{E}(x)\right] \cdot \mathrm{a}_{j}^{*}\right) .
\end{aligned}
$$

By the convexity of the exponential function and the fact that $a_{1}^{*}+a_{2}^{*}+a_{3}^{*}=0$, we have $\gamma_{i}^{ \pm}, \delta_{i}^{ \pm} \geqq 3$; in addition, $\sqrt{\left(\gamma_{i}^{+}-\gamma_{i}^{-}\right)^{2}+4 \delta_{i}^{+} \delta_{i}^{-}}+\gamma_{i}^{+}-\gamma_{i}^{-} \geqq 0$, so $h_{f, i}^{ \pm}(x)$ is well-defined for all $x$. Since $\gamma_{i}^{ \pm}$and $\delta_{i}^{ \pm}$are continuous functions of $x \in \mathscr{M}_{\infty}^{\varepsilon}$, we also have that $h_{f, i}^{ \pm}(x)$ depend continuously on $x$, and is thus uniformly bounded for $x \in \mathscr{M}_{\infty}^{\varepsilon}$.

By now expressing $g(x)$ in terms of hyperbolic trigonometric functions, we define $H f(x):=\mathcal{H}_{A, B}^{\operatorname{Tr}}(x, \nabla f(x))$, where the limiting Hamiltonian $\mathcal{H}_{A, B}^{\operatorname{Tr}}(x, p)$ is defined to be

$$
\begin{aligned}
\mathcal{H}_{A, B}^{\mathrm{Tr}}(x, p):= & \sum_{i=1}^{m} \sqrt{\Upsilon_{A, B}\left[\partial_{i} \mathcal{E}(x)\right]^{2}+\Psi_{A, B}^{\mathrm{Tr}}\left[\frac{1}{B} p_{i}-\partial_{i} \mathcal{E}(x)\right]-\Psi_{A, B}^{\mathrm{Tr}}\left[-\partial_{i} \mathcal{E}(x)\right]} \\
& -\Upsilon_{A, B}\left[\partial_{i} \mathcal{E}(x)\right],
\end{aligned}
$$

for $x \in \mathscr{M}_{\infty}^{\varepsilon} \backslash \partial \mathscr{M}_{\infty}^{\varepsilon}$, and $\mathcal{H}_{A, B}^{\mathrm{Tr}}(x, p):=0$ for $x \in \partial \mathscr{M}_{\infty}^{\varepsilon}$, where

$$
\Psi_{A, B}^{\operatorname{Tr}}[\xi]:=A^{2} \sum_{j=1}^{6} \cosh \left[B \xi_{i} \cdot \mathrm{a}_{j}\right], \quad \text { and } \Upsilon_{A, B}[\xi]:=A \sum_{j=1}^{3} \cosh \left[B \xi_{i} \cdot \mathrm{a}_{j}^{*}\right]
$$


We define the conjugate function $\mathcal{L}_{A, B}^{\mathrm{Tr}}: \mathscr{M}_{\infty}^{\varepsilon} \times \mathbb{R}^{2 m} \rightarrow \mathbb{R} \cup\{+\infty\}$ to be

$$
\mathcal{L}_{A, B}^{\operatorname{Tr}}(x, \xi):=\sup _{p \in \mathbb{R}^{2 m}}\left\{\xi \cdot p-\mathcal{H}_{A, B}^{\operatorname{Tr}}(x, p)\right\}
$$

and the corresponding rate functional $\mathcal{J}: \mathrm{D}\left([0,+\infty) ; \mathscr{M}_{\infty}^{\varepsilon}\right) \rightarrow \mathbb{R} \cup\{+\infty\}$ to be

$$
\mathcal{J}_{A, B}^{\operatorname{Tr}}(x):= \begin{cases}\int_{0}^{\infty} \mathcal{L}_{A, B}^{\operatorname{Tr}}(x, \dot{x}) \mathrm{d} t & x \in \mathrm{W}^{1,1}\left([0,+\infty) ; \mathbb{R}^{2 m}\right), \\ +\infty & \text { otherwise. }\end{cases}
$$

We now state the following theorem, which asserts the existence of a Large Deviations Principle for the model for dislocation motion for the case $L=\operatorname{Tr}$.

Lemma 6.2. Suppose that $\mathrm{L}=\operatorname{Tr}$, and that $X_{0}^{n}=\iota_{n}\left(x^{n}\right)$ where $x^{n} \rightarrow x_{0} \in \mathscr{M}_{\infty}^{\varepsilon}$ as $n \rightarrow \infty$. Then the processes $X_{t}^{n}$ satisfy a Large Deviations Principle with good rate function $\mathcal{J}_{A, B}^{\operatorname{Tr}}$.

Once more, we prove this result by checking the conditions of Theorem 3.3.

Proof. As in the proof of Lemma 6.1, conditions (1) and (2) are straightforward to verify with $M_{n}=\mathscr{M}_{n}^{\varepsilon}$ and $M=\mathscr{M}_{\infty}^{\varepsilon}$, and condition (3) holds by an identical argument.

Verifying Condition (4) It is clear from the arguments of the previous section that $\mathcal{H}_{A, B}^{\operatorname{Tr}}(x, p)$ satisfies the necessary regularity conditions, and by definition the $\mathcal{H}_{A, B}^{\mathrm{Tr}}$ vanishes for $x \in \partial \mathscr{M}_{\infty}^{\varepsilon}$; the convexity condition is also evident for $x \in \partial \mathscr{M}_{\infty}^{\varepsilon}$. Next, let $x$ lie in the interior of $\mathscr{M}_{\infty}^{\varepsilon}$ : then the second derivative of $\mathcal{H}_{A, B}^{\mathrm{Tr}}(x, p)$ with respect to $p_{i}$ is

$$
\begin{aligned}
\partial_{p_{i}}^{2} \mathcal{H}_{A, B}^{\mathrm{Tr}}(x, p) & =\frac{\frac{1}{2 B^{2}} \nabla^{2} \Psi_{A, B}^{\mathrm{Tr}}\left[\frac{1}{B} p_{i}-\partial_{i} \mathcal{E}(x)\right]}{\left(\Upsilon_{A, B}\left[\partial_{i} \mathcal{E}(x)\right]^{2}+\Psi_{A, B}^{\mathrm{Tr}}\left[\frac{1}{B} p_{i}-\partial_{i} \mathcal{E}(x)\right]-\Psi_{A, B}^{\mathrm{Tr}}\left[-\partial_{i} \mathcal{E}(x)\right]\right)^{1 / 2}} \\
& -\frac{\frac{1}{4 B^{2}} \nabla \Psi_{A, B}^{\mathrm{Tr}}\left[\frac{1}{B} p_{i}-\partial_{i} \mathcal{E}(x)\right] \otimes \nabla \Psi_{A, B}^{\mathrm{Tr}}\left[\frac{1}{B} p_{i}-\partial_{i} \mathcal{E}(x)\right]}{\left(\Upsilon_{A, B}\left[\partial_{i} \mathcal{E}(x)\right]^{2}+\Psi_{A, B}^{\mathrm{Tr}}\left[\frac{1}{B} p_{i}-\partial_{i} \mathcal{E}(x)\right]-\Psi_{A, B}^{\mathrm{Tr}}\left[-\partial_{i} \mathcal{E}(x)\right]\right)^{3 / 2}} .
\end{aligned}
$$

To verify convexity of $\mathcal{H}_{A, B}^{\mathrm{Tr}}$, we check that this matrix is positive definite. This reduces to verifying that, as symmetric matrices, $\frac{1}{2}\left(\Upsilon_{A, B}[\zeta]^{2}-\Psi_{A, B}^{\operatorname{Tr}}[\zeta]+\Psi_{A, B}^{\operatorname{Tr}}[\xi]\right) \nabla^{2} \Psi_{A, B}^{\operatorname{Tr}}[\xi]-\frac{1}{4} \nabla \Psi_{A, B}^{\operatorname{Tr}}[\xi] \otimes \nabla \Psi_{A, B}^{\operatorname{Tr}}[\xi] \geqq 0$

for all $\xi, \zeta \in \mathbb{R}^{2}$. Pre-multiplying by $v^{T}$ and post-multiplying the matrices in the above expression by $v$ for some $v \in \mathbb{R}^{2}$, we have

$$
\begin{aligned}
\nabla^{2} \Psi_{A, B}^{\operatorname{Tr}}[\xi]:[v, v] & =\sum_{j=1}^{6} A^{2} B^{2} \cosh \left[B \xi \cdot \mathrm{a}_{j}\right]\left(v \cdot \mathrm{a}_{j}\right)^{2} \\
\text { and } \quad\left(v \cdot \nabla \Psi_{A, B}^{\operatorname{Tr}}[\xi]\right)^{2} & =\left(\sum_{i=1}^{6} A^{2} B \sinh \left[B \xi \cdot \mathrm{a}_{j}\right]\left(v \cdot \mathrm{a}_{j}\right)\right)^{2} .
\end{aligned}
$$


It is immediate that $\nabla^{2} \Psi_{A, B}^{\operatorname{Tr}}[\xi]:[v, v] \geqq 0$ for all $v \in \mathbb{R}^{2}$, since cosh is bounded below by 1 , and the vectors $\mathrm{a}_{j}$ span $\mathbb{R}^{2}$. Next, we note that

$$
\begin{aligned}
\frac{1}{2} \Psi_{A, B}^{\operatorname{Tr}}[\xi] \nabla^{2} \Psi_{A, B}^{\operatorname{Tr}}[\xi]:[v, v]-\frac{1}{4}\left(v \cdot \nabla \Psi_{A, B}^{\operatorname{Tr}}[\xi]\right)^{2} \\
=\frac{1}{2} \sum_{j, k=1}^{6} A^{4} B^{2} \cosh \left[B \xi \cdot \mathrm{a}_{j}\right] \cosh \left[B \xi \cdot \mathrm{a}_{k}\right]\left(v \cdot \mathrm{a}_{j}\right)^{2} \\
\quad-\frac{1}{4} \sum_{j, k=1}^{6} A^{4} B^{2} \sinh \left[B \xi \cdot \mathrm{a}_{j}\right] \sinh \left[B \xi \cdot \mathrm{a}_{j}\right]\left(v \cdot \mathrm{a}_{j}\right)\left(v \cdot \mathrm{a}_{k}\right) .
\end{aligned}
$$

Using the identity $\left(v \cdot \mathrm{a}_{j}\right)\left(v \cdot \mathrm{a}_{k}\right)=\frac{1}{2}\left[v \cdot\left(\mathrm{a}_{j}+\mathrm{a}_{k}\right)\right]^{2}-\frac{1}{2}\left(v \cdot \mathrm{a}_{j}\right)^{2}-\frac{1}{2}\left(v \cdot \mathrm{a}_{k}\right)^{2}$ and the symmetry of the vectors $a_{j}$, we have

$$
\begin{aligned}
& \frac{1}{4} \sum_{j, k=1}^{6} \sinh \left[B \xi \cdot \mathrm{a}_{j}\right] \sinh \left[B \xi \cdot \mathrm{a}_{k}\right]\left(v \cdot \mathrm{a}_{j}\right)\left(v \cdot \mathrm{a}_{k}\right) \\
& =\frac{1}{8} \sum_{j, k=1}^{6} \sinh \left[B \xi \cdot \mathrm{a}_{j}\right] \sinh \left[B \xi \cdot \mathrm{a}_{k}\right]\left[v \cdot\left(\mathrm{a}_{j}+\mathrm{a}_{k}\right)\right]^{2} \\
& \quad-\frac{1}{4} \sum_{j, k=1}^{6} \sinh \left[B \xi \cdot \mathrm{a}_{j}\right] \sinh \left[B \xi \cdot \mathrm{a}_{k}\right]\left(v \cdot \mathrm{a}_{j}\right)^{2} .
\end{aligned}
$$

The latter sum vanishes, since sinh is an odd function and $v \cdot \mathrm{a}_{k}=-v \cdot \mathrm{a}_{k+3}$ for $k=1,2$ or 3. Splitting the sum, interchanging indices $j$ and $k$ and then using convexity and the fact that cosh is postive, we have

$$
\begin{aligned}
& \frac{1}{2} \sum_{j, k=1}^{6} \cosh \left[B \xi \cdot \mathrm{a}_{j}\right] \cosh \left[B \xi \cdot \mathrm{a}_{k}\right]\left(v \cdot \mathrm{a}_{j}\right)^{2} \\
& \quad=\frac{1}{2} \sum_{j, k=1}^{6} \cosh \left[B \xi \cdot \mathrm{a}_{j}\right] \cosh \left[B \xi \cdot \mathrm{a}_{k}\right]\left(\frac{1}{2}\left(v \cdot \mathrm{a}_{j}\right)^{2}+\frac{1}{2}\left(v \cdot \mathrm{a}_{k}\right)^{2}\right) \\
& \quad \geqq \frac{1}{8} \sum_{j, k=1}^{6} \cosh \left[B \xi \cdot \mathrm{a}_{j}\right] \cosh \left[B \xi \cdot \mathrm{a}_{k}\right]\left[v \cdot\left(\mathrm{a}_{j}+\mathrm{a}_{k}\right)\right]^{2} .
\end{aligned}
$$

Combining (6.4) and (6.5), and using the addition formula for hyperbolic cosine, then bounding cosh below by 1 and dropping all terms except for those where $j=k$, we find that

$$
\begin{aligned}
& \frac{1}{2} \Psi_{A, B}^{\operatorname{Tr}}[\xi] \nabla^{2} \Psi_{A, B}^{\operatorname{Tr}}[\xi]:[v, v]-\frac{1}{4}(v \cdot \nabla \Psi[\xi])^{2} \\
& \geqq \frac{1}{8} A^{4} B^{2} \sum_{j, k=1}^{6} \cosh \left[B \xi \cdot\left(\mathrm{a}_{j}+\mathrm{a}_{k}\right)\right]\left[v \cdot\left(\mathrm{a}_{j}+\mathrm{a}_{k}\right)\right]^{2}, \\
& \geqq \frac{1}{2} A^{4} B^{2} \sum_{j=1}^{6}\left(v \cdot \mathrm{a}_{j}\right)=\frac{3}{2} A^{4} B^{2}|v|^{2}
\end{aligned}
$$


It remains to verify that $\left(\Upsilon_{A, B}[\zeta]^{2}-\Psi_{A, B}^{\operatorname{Tr}}[\zeta]\right) \frac{1}{2} \nabla^{2} \Psi_{A, B}^{\operatorname{Tr}}[\xi] \geqq 0$ for all $\xi, \zeta \in \mathbb{R}^{2}$. This is immediate upon noting that

$$
\begin{aligned}
\Upsilon_{A, B}[\zeta]^{2}-\Psi_{A, B}^{\operatorname{Tr}}[\zeta] & =\frac{1}{4}\left(\gamma_{i}^{+}+\gamma_{i}^{-}\right)^{2}-\gamma_{i}^{+} \gamma_{i}^{-}=\frac{1}{4}\left(\gamma_{i}^{+}-\gamma_{i}^{-}\right)^{2} \\
& =\left(\sum_{j=1}^{6} A \sinh \left[B \xi \cdot \mathrm{a}_{j}\right]\right)^{2} \geqq 0
\end{aligned}
$$

and using the positive-definiteness of $\nabla^{2} \Psi_{A, B}^{\mathrm{Tr}}[\xi]$. Estimates (6.6) and (6.7) entail that $\partial_{p_{i}}^{2} \mathcal{H}_{A, B}^{\mathrm{Tr}}(x, p)$ is strictly positive definite for all $x$ in the interior of $\mathscr{M}_{\infty}^{\varepsilon}$, and therefore $\mathcal{H}_{A, B}^{\mathrm{Tr}}$ satisfies the convexity condition.

To verify that the convergence requirement of Condition (4) is satisfied, we define $h_{n}: \mathscr{M}_{n}^{\varepsilon} \rightarrow \mathbb{R}$ to be $h_{n}(\mu):=h\left(\iota_{n}(\mu), \mu\right)$. Then as $h_{f}\left(x, e_{1}^{*}, \ldots, e_{m}^{*}\right)$ is uniformly bounded for all $x \in \mathscr{M}_{\infty}^{\varepsilon}$ and $e_{i}^{*} \in \operatorname{Tr}^{*}$, so

$$
\left\|f \circ \iota_{n}+\frac{1}{n} h_{n}-f \circ \iota_{n}\right\| \leqq c n^{-1} \rightarrow 0 \quad \text { as } n \rightarrow \infty \text {. }
$$

Since $\nabla f, \partial_{i} \mathcal{E}$ and $x \mapsto h_{f}(x, \mu)$ are uniformly continuous on $\mathscr{M}_{\infty}^{\varepsilon}$, and $\mathcal{H}_{A, B}^{\mathrm{Tr}}$ is smooth and hence uniformly continuous on the interior of $\mathscr{M}_{\infty}^{\varepsilon} \times B_{r}(0)$ for any $r>0$, we have that $x \mapsto \mathcal{H}_{A, B}^{\mathrm{Tr}}(x, \nabla f(x))$ is uniformly continuous. Using the fact that $h_{f}$ was chosen to satisfy (6.3), it is now straightforward to check that

$$
\left\|H_{n}\left(f \circ \iota_{n}+\frac{1}{n} h_{n}\right)(\mu)-H f \circ \iota_{n}\right\| \rightarrow 0 \text { as } n \rightarrow \infty,
$$

and so convergence is verified.

Verifying Condition (5) Given that $\mathcal{H}_{A, B}^{\mathrm{Tr}}$ is a significantly more complex function than the Hamiltonians obtained in the previous cases, we do not have as explicit an expression for $\mathcal{L}_{A, B}^{\mathrm{Tr}}$ as we obtained in the cases where $\mathrm{L}=\mathrm{Sq}$ and $\mathrm{L}=\mathrm{Hx}$. We therefore verify Condition (5) indirectly using properties of the Legendre-Fenchel transform.

First, we verify that $\mathcal{L}_{A, B}^{\mathrm{Tr}}(x, \xi) \geqq 0$. We note that since $\mathcal{H}_{A, B}^{\mathrm{Tr}}(x, p)$ is smooth and strictly convex in $p, \mathcal{L}_{A, B}^{\operatorname{Tr}}(x, \xi)$ is also smooth and strictly convex, and $\mathcal{H}_{A, B}^{\mathrm{Tr}}(x, p)=\sup _{\xi \in \mathbb{R}^{2 m}}\left\{p \cdot \xi-\mathcal{L}_{A, B}^{\mathrm{Tr}}(x, \xi)\right\}$. It follows that

$$
0=\mathcal{H}_{A, B}^{\operatorname{Tr}}(x, 0)=\sup _{\xi \in \mathbb{R}^{2 m}}\left\{-\mathcal{L}_{A, B}^{\operatorname{Tr}}(x, \xi)\right\}=-\inf _{\xi \in \mathbb{R}^{2 m}} \mathcal{L}_{A, B}^{\operatorname{Tr}}(x, \xi)
$$

To verify the growth condition (3.9), we estimate $\mathcal{H}_{A, B}^{\mathrm{Tr}}(x, p)$ above. Using the elementary inequality $\sqrt{a+b} \leqq \sqrt{a}+\sqrt{b}$ for any $a, b \geqq 0$, the AM-GM inequality, and the property that $\gamma_{i}^{ \pm} \geqq 3$, we find

$$
\begin{aligned}
\sqrt{\frac{1}{4}\left(\gamma_{i}^{+}-\gamma_{i}^{-}\right)^{2}+\delta_{i}^{+} \delta_{i}^{-}}-\frac{1}{2}\left(\gamma_{i}^{+}+\gamma_{i}^{-}\right) & \leqq \frac{1}{2}\left|\gamma_{i}^{+}-\gamma_{i}^{-}\right|+\sqrt{\delta_{i}^{+} \delta_{i}^{-}}-\frac{1}{2}\left(\gamma_{i}^{+}+\gamma_{i}^{-}\right) \\
& \leqq \frac{1}{2}\left(\delta_{i}^{+}+\delta_{i}^{-}\right)-\min \left\{\gamma_{i}^{+}, \gamma_{i}^{-}\right\} \\
& \leqq \frac{1}{2}\left(\delta_{i}^{+}+\delta_{i}^{-}\right)
\end{aligned}
$$


Noting that $\cosh \left(v \cdot \mathrm{a}_{j}\right) \leqq \cosh \left(\frac{\sqrt{3}}{3}|v|\right)$, formula (6.8), along with the definition of $\mathcal{H}_{A, B}^{\mathrm{Tr}}$, the convexity of cosh and the fact that $\partial_{i} \mathcal{E}(x)$ is uniformly bounded for all $x \in \mathscr{M}_{\infty}^{\varepsilon}$, implies that there exists a constant $C>0$ independent of $x$ such that $\mathcal{H}_{A, B}^{\operatorname{Tr}}(x, p) \leqq \sum_{i=1}^{m} \sum_{j=1}^{3} A \cosh \left(\left[p_{i}-B \partial_{i} \mathcal{E}(x)\right] \cdot \mathrm{a}_{j}^{*}\right) \leqq \sum_{i=1}^{m} \frac{3}{2} A \cosh \left[\frac{\sqrt{3}}{3}\left|p_{i}\right|\right]+C$.

A similar argument to that used in the proof of Theorem 6.1 now allows us to conclude that (3.9) also holds in this case.

Next, we note that

$$
0=\partial_{\xi} \mathcal{L}_{A, B}^{\operatorname{Tr}}(x, \xi) \text { if and only if } \xi=\partial_{p} \mathcal{H}_{A, B}^{\operatorname{Tr}}(x, 0) .
$$

Computing $\partial_{p} \mathcal{H}_{A, B}^{\mathrm{Tr}}$, we find that if $x$ solves

$$
\dot{x}_{i}=\frac{\nabla \Psi_{A, B}^{\operatorname{Tr}}\left[-\partial_{i} \mathcal{E}(x)\right]}{2 \Upsilon_{A, B}\left[\partial_{i} \mathcal{E}(x)\right]} \text { with } \quad x(0)=x_{0},
$$

where $x_{0}$ is in the interior of $\mathscr{M}_{\infty}^{\varepsilon}$, then (3.10) is verified. As $\Psi_{A, B}^{\operatorname{Tr}}$ and $\Upsilon_{A, B}$ are smooth, $\Upsilon_{A, B}$ is bounded below, and $\partial \mathcal{E}(x)$ is bounded on $\mathscr{M}_{\infty}^{\varepsilon}$, an identical argument to that given in the proof of Theorem 6.1 entails that this condition is satisfied.

Having now verified all conditions of Theorem 3.3, its application implies Lemma 6.2.

Finally, upon noting that $\mathcal{L}_{A, B}^{\operatorname{Tr}}$ is minimised when (6.9) is satisfied, and setting

$$
\mathcal{M}_{A, B}^{\mathrm{Tr}}(\xi)=\frac{\nabla \Psi_{A, B}^{\mathrm{Tr}}[\xi]}{2 \Upsilon_{A, B}[\xi]}
$$

we have proved Theorem 3.4.

Acknowledgements. The author would like to thank Giovanni Bonaschi and Giacomo Di Gesú for informative discussions on Large Deviations Principles while carrying out this work, and the two anonymous referees for helpful suggestions of a variety of improvements to this paper.

Funding This study was funded by a public grant overseen by the French National Research Agency (ANR) as part of the "Investissements d'Avenir" program (reference: ANR10-LABX-0098).

Conflict of interest The author declares that there is no conflict of interest regarding this work.

Open Access This article is distributed under the terms of the Creative Commons Attribution 4.0 International License (http://creativecommons.org/licenses/by/4.0/), which permits unrestricted use, distribution, and reproduction in any medium, provided you give appropriate credit to the original author(s) and the source, provide a link to the Creative Commons license, and indicate if changes were made. 


\section{References}

1. Alicandro, R., De Luca, L., Garroni, A., Ponsiglione, M.: Dynamics of discrete screw dislocations on glide directions. J. Mech. Phys. Solids 92, 87-104 (2016)

2. Alicandro, R., De Luca, L., Garroni, A., Ponsiglione, M.: Metastability and dynamics of discrete topological singularities in two dimensions: a $\Gamma$-convergence approach. Arch. Ration. Mech. Anal. 214(1), 269-330 (2014)

3. Amodeo, R.J., Ghoniem, N.M.: Dislocation dynamics. I. A proposed methodology for deformation micromechanics. Phys. Rev. B 41(10), 6958 (1990)

4. Ariza, M.P., Ortiz, M.: Discrete crystal elasticity and discrete dislocations in crystals. Arch. Ration. Mech. Anal. 178(2), 149-226 (2005)

5. Ariza, M.P., Ortiz, M.: Discrete dislocations in graphene. J. Mech. Phys. Solids 58(5), 710-734 (2010)

6. ArrheniUS, S.: Über die Reaktionsgeschwindigkeit bei der Inversion von Rohrzucker durch Säuren. Zeitschrift für physikalische Chemie 4, 226-248 (1889)

7. BERGLund, N.: Kramers' law: validity, derivations and generalisations. Markov Process. Relat. Fields 19(3), 459-490 (2013)

8. Boateng, H.A., Schulze, T.P., Smereka, P.: Approximating off-lattice kinetic Monte Carlo. Multiscale Model. Simul. 12(1), 181-199 (2014)

9. Bollmann, W.: Interference effects in the electron microscopy of thin crystal foils. Phys. Rev. 103(5), 1588 (1956)

10. Bonaschi, G.A., Peletier, M.A.: Quadratic and rate-independent limits for a largedeviations functional. Contin. Mech. Thermodyn. 28(4),1191-1219 (2016)

11. Bovier, A., Gayrard, V.: Sample path large deviations for a class of Markov chains related to disordered mean field models. WIAS preprints, 1999. Preprint No. 487

12. Bulatov, V.V., CaI, W.: Computer Simulations of Dislocations, volume 3 of Oxford Series on Materials Modelling. Oxford University Press, Oxford, 2006

13. CaI, W., Bulatov, V.V.: Mobility laws in dislocation dynamics simulations. Mater. Sci. Eng. A 387, 277-281 (2004)

14. Cermelli, P., Leoni, G.: Renormalized energy and forces on dislocations. SIAM J. Math. Anal. 37(4), 1131-1160 (2005) (electronic)

15. Crandall, M.G., Ishit, H., Lions, P.-L.: User's guide to viscosity solutions of second order partial differential equations. Bull. Am. Math. Soc. (N.S.) 27(1), 1-67 (1992)

16. Dembo, A., Zeitouni, O.: Large deviations techniques and applications, volume 38 of Stochastic Modelling and Applied Probability. Springer, Berlin, 2010. Corrected reprint of the second (1998) edition

17. Ehrlacher, V., Ortner, C., Shapeev, A.V.: Analysis of boundary conditions for crystal defect atomistic simulations. Arch. Rational. Mech. Anal. 222, 1217-1268 (2016). doi:10.1007/s00205-016-1019-6

18. Ellis, R.S.: Entropy, Large Deviations, and Statistical Mechanics. Classics in Mathematics. Springer, Berlin, 2006. Reprint of the 1985 original

19. Evans, L.C., GARIEPY, R.F.: Measure Theory and Fine Properties of Functions. Studies in Advanced Mathematics. CRC Press, Boca Raton, 1992

20. Eyring, H.: The activated complex in chemical reactions. J. Chem. Phys. 3(2), 107-115 (1935)

21. Feng, J., Kurtz, T.G.: Large Deviations for Stochastic Processes, volume 131 of Mathematical Surveys and Monographs. American Mathematical Society, Providence, 2006

22. Freidlin, M.I., Wentzell, A.D.: Random Perturbations of Dynamical Systems, volume 260 of Grundlehren der Mathematischen Wissenschaften [Fundamental Principles of Mathematical Sciences], 3rd edn. Springer, Heidelberg, 2012. Translated from the 1979 Russian original by Joseph Szücs

23. Garnett, J.B., Marshall, D.E.: Harmonic Measure, volume 2 of New Mathematical Monographs. Cambridge University Press, Cambridge, 2008. Reprint of the 2005 original 
24. Gilbarg, D., Trudinger, N.S.: Elliptic Partial Differential Equations of Second Order. Classics in Mathematics. Springer, Berlin, 2001. Reprint of the 1998 edition

25. Grisvard, P.: Elliptic Problems in Nonsmooth Domains, volume 69 of Classics in Applied Mathematics. SIAM, Philadelphia, 2011

26. GuAdie, M.A.: Harmonic Functions On Square Lattices: Uniqueness Sets and Growth Properties. Ph.D. thesis, Norwegian University of Science and Technology, Trondheim, 2013

27. HÄNGGi, P., TAlKner, P., Borkovec, M.: Reaction-rate theory: fifty years after Kramers. Rev. Mod. Phys., 62, 251-341 (1990)

28. Hatcher, A.: Algebraic Topology. Cambridge University Press, Cambridge, 2002

29. Hirsch, P.B., Horne, R.W., Whelan, M.J.: LXVIII. Direct observations of the arrangement and motion of dislocations in aluminium. Philos. Mag. 1(7), 677-684 (1956)

30. Hirth, J.P., Lothe, J.: Theory of Dislocations. Krieger Publishing Company, Malabar, 1982

31. Hudson, T., Ortner, C.: Analysis of stable screw dislocation configurations in an antiplane lattice model. SIAM J. Math. Anal. 47(1), 291-320 (2015)

32. Hudson, T., Ortner, C.: Existence and stability of a screw dislocation under anti-plane deformation. Arch. Ration. Mech. Anal. 213(3), 887-929 (2014)

33. Hull, D., BACON, D.J.: Introduction to Dislocations, vol. 37. Butterworth-Heinemann, Oxford, 2011

34. KRAmers, H.A.: Brownian motion in a field of force and the diffusion model of chemical reactions. Physica 7, 284-304 (1940)

35. Lawler, G.F., Limic, V.: Random Walk: A Modern Introduction, volume 123 of Cambridge Studies in Advanced Mathematics. Cambridge University Press, Cambridge, 2010

36. Mielke, A., Peletier, M.A., Renger, D.R.M.: On the relation between gradient flows and the large-deviation principle, with applications to Markov chains and diffusion. Potential Anal. 41(4), 1293-1327 (2014)

37. Mielke, A.: On evolutionary $\Gamma$-convergence for gradient systems. Macroscopic and Large Scale Phenomena: Coarse Graining, Mean Field Limits and Ergodicity (Eds. Muntean A., Rademacher J. and Zagaris A.) Springer International Publishing, Cham, $187-249,2016$

38. Munkres, J.R.: Elements of Algebraic Topology. Addison-Wesley Publishing Company, Menlo Park, 1984

39. Orowan, E.: Zur Kristallplastizität. III. Zeitschrift für Physik 89, 634-659 (1934)

40. Polanyi, M.: Über eine Art Gitterstörung, die einen Kristall plastisch machen könnte. Zeitschrift für Physik 89, 660-664 (1934)

41. Ponsiglione, M.: Elastic energy stored in a crystal induced by screw dislocations: from discrete to continuous. SIAM J. Math. Anal. 39(2), 449-469 (2007)

42. Rockafellar, R.T.: Convex Analysis. Princeton Landmarks in Mathematics. Princeton University Press, Princeton, 1997. Reprint of the 1970 original, Princeton Paperbacks

43. Sandier, E., Serfaty, S.: From the Ginzburg-Landau model to vortex lattice problems. Commun. Math. Phys. 313(3), 635-743 (2012)

44. Schulze, T.P., Smereka, P., Weinan, E.: Coupling kinetic Monte-Carlo and continuum models with application to epitaxial growth. J. Comput. Phys. 189(1), 197-211 (2003)

45. TAYlOR, G.I.: The mechanism of plastic deformation of crystals. Part I. Theoretical. Proc. R. Soc. Lond. Ser. A Contain. Pap. Math. Phys. Character 145(855), 362-387 (1934)

46. VAn der Giessen, E., Needleman, A.: Discrete dislocation plasticity: a simple planar model. Model. Simul. Mater. Sci. Eng. 3(5), 689 (1995)

47. Voter, A.F.: Introduction to the kinetic Monte Carlo method. In: Sickafus, K.E., Kotomin, E.A., Uberuaga, B.P. (eds) Radiation Effects in Solids Springer, Berlin, 1-23, 2007 
T. Hudson

Mathematics Institute

University of Warwick

Zeeman Building

Coventry

CV4 7AL

UK.

e-mail: t.hudson.1@warwick.ac.uk

(Received October 10, 2015 / Accepted January 16, 2017)

Published online February 4, 2017 - () The Author(s) (2017)

This article is published with open access at Springerlink.com 